\title{
SEARCH TECHNIQUES FOR CHARGED AND NEUTRAL INTERMEDIATE-MASS HIGGS BOSONS*
}

\author{
J.F. GUNION \\ Department of Physics, U.C. Davis, Davis, CA 95616, USA \\ G.L. KANE and Jose WUDKA \\ Randall Laboratory of Physics, University of Michigan, Ann Arbor, MI 48104, USA
}

Received 12 October 1987

\begin{abstract}
If fundamental scalar bosons exist, a natural implication of theories based on supersymmetry or superstrings, Higgs boson masses are likely to be of order $m_{Z}$ within roughly a factor of two. We term this the intermediate mass range. We study how such Higgs bosons could be detected at $\mathrm{e}^{+} \mathrm{e}^{-}$and, particularly, at hadron colliders. At a hadron collider it appears to be necessary to use rare decay modes of the Higgs boson, and we examine $\mathrm{H}^{0} \rightarrow \gamma \gamma, \tau^{+} \tau^{-}, \mathrm{Z} \gamma, \mathrm{Z} \ell^{+} \ell^{-}, \Theta \gamma$, and $\mathrm{b} \overline{\mathrm{b}}$. Since charged scalars also occur in all theories beyond the minimal Standard Model, we examine ways to find the $\mathrm{H}^{ \pm}$, concentrating on $\mathrm{H}^{ \pm} \rightarrow \tau^{ \pm} \nu, \mathrm{W}^{ \pm} \mathrm{H}^{0}, \mathrm{~W}^{ \pm} \mathrm{H}^{0} \gamma, \mathrm{W}^{ \pm} \gamma$, and $\mathrm{W}{ }^{ \pm} \mathrm{Z}$. Because obtaining data about the spectrum of Higgs bosons is so essential, our approach is to ask what facilities would be sufficient to cover the entire intermediate mass range.
\end{abstract}

\section{Introduction}

Although the Higgs mechanism is technically satisfactory to introduce gauge boson and fermion masses in the standard model (SM), it is still not understood physically, even two decades after it was introduced in particle physics. Some theorists believe [1] that no elementary scalar bosons (Higgs bosons) will exist. Others, motivated by the belief that the standard model will be made conceptually complete on the $\mathrm{TeV}$ scale by being part of a supersymmetric theory, expect point-like scalars to be as much a part of the particle spectrum as fermions and gauge bosons. If either supersymmetry or superstring based theories are relevant to understanding the standard model, mass bounds on the lightest neutral scalar place it in the intermediate mass range.

To make progress in unraveling the physics of spontaneous symmetry breaking it is probably essential to completely measure the spectrum of scalar bosons, both neutral

* Work supported, in part, by the Department of Energy. 
and charged. Certainly knowledge of the Higgs boson spectrum will be required in order to test both existing and yet undeveloped theoretical ideas. We note that measuring the spectrum also implies a determination of those mass regions that contain no spin zero bosons.

For Higgs masses below $\sim 0.5 m_{\mathrm{Z}}$, which we call light Higgs bosons, a complete search can be carried out at SLC, LEP, and existing machines, for both neutral and charged Higgs scalars. This limit could be extended if toponium $(\Theta)$ occurs at a mass accessible to these machines. If LEP-II is constructed, the accessible mass range for a neutral Higgs can be extended to $\$ 85 \mathrm{GeV}$ [2]. However, since LEP-II is not currently scheduled to take data until 1994, and decisions could be made that would delay it, we will not assume that it will be available. Thus, we will attempt to find techniques for detection at a hadron machine of both charged and neutral scalars with masses above about $m_{\mathrm{Z}} / 2$.

In our discussions it will be useful to consider results both for the standard model, with one neutral scalar, which we denote by $\mathrm{H}_{\mathrm{SM}}^{0}$, and for a two-doublet Higgs sector, which yields five physical Higgs bosons: a light neutral scalar, $\mathbf{h}$; a heavier neutral scalar, $\mathrm{H}$; a neutral pseudoscalar, A; and two charged Higgs bosons, denoted by $\mathrm{H}^{ \pm}$. (The terms "scalar" and "pseudoscalar" refer to the way in which the neutral Higgs bosons couple to fermion pairs.) A generic neutral scalar boson will be denoted simply by $\mathrm{H}^{0}$.

For the neutral scalar boson of the minimal SM, once $m_{\mathrm{H}_{\mathrm{SM}}^{0}}>2 m_{\mathrm{Z}}$ the search $[3,4]$ is quite straightforward at the SSC until $m_{\mathbf{H}_{S M}^{0}}$ of about $0.5-0.6 \mathrm{TeV}$. The production cross section is large, and the decay $\mathrm{H}_{\mathrm{SM}}^{0} \rightarrow \mathrm{ZZ}$, followed by both Z's decaying to $\ell^{+} \ell^{-}$, provides a signature which is good for triggering and essentially background free. Various strategies can probably extend this range by another 0.2 $\mathrm{TeV}$. Similar remarks would hold for any high-luminosity, multi-TeV hadron collider. Scalar bosons in the above mass range we term heavy Higgs bosons.

If the SM Higgs boson has mass above $\sim 0.7-0.8 \mathrm{TeV}$ (we refer to such a Higgs as obese) it is necessary to study WW scattering [5] to extract the scalar interaction. The theoretical analyses required for this purpose are available. Both hadron and $\mathrm{e}^{+} \mathrm{e}^{-}$colliders produce "beams" of $\mathrm{W}$ bosons [6]. The tree-level gauge invariant cross sections for WW $\rightarrow$ WW scattering, including Higgs boson contributions, have been calculated and provide the standard of comparison [7] to determine whether the WW scattering is perturbative (which it would be if light or intermediate mass Higgs bosons exist) or not. For more detailed analyses the complete tree-level calculation of $\mathrm{ff} \rightarrow \mathrm{ffWW}$ has also been done [8,9], allowing a full investigation of the effects of various types of triggers and procedures for eliminating backgrounds. Extensive and fairly systematic theoretical studies of backgrounds have been done [10-14]. Reasonably realistic simulations including detector effects have not been completed but are underway [15]. In the near future, the energies and luminosities required in order to study WW scattering in the TeV range at $\mathrm{e}^{+} \mathrm{e}^{-}$and hadron colliders will be known [16]. 
In this paper we will focus on the region of intermediate masses, approximately $0.5 m_{\mathrm{Z}} \leqq m_{\mathrm{H}^{0}} \leqq 2 m_{\mathrm{Z}}$. Previous studies of this region are available [17-21]. At $\mathrm{e}^{+} \mathrm{e}^{-}$ colliders the dominant $\mathrm{H}^{0}$ decay modes, $\mathrm{H}^{0} \rightarrow \mathrm{tt}$ or $\mathrm{H}^{+} \rightarrow \bar{t} \bar{b}$, can be used $[18,21]$, since backgrounds are not too large, and we do not have much to add to previous work. We note that the intermediate mass region could be covered by a machine that is a modest extension above SLC and LEP. An $\mathrm{e}^{+} \mathrm{e}^{-}$collider with $\sqrt{s} \geq 325$ $\mathrm{GeV}$ and $\mathscr{L} \geq 2 \times 10^{32} \mathrm{~cm}^{-2} \mathrm{sec}^{-1}$ would be sufficient, though somewhat larger values of these parameters would be required to study in detail the properties of any scalar boson that was discovered. Since the region above $2 m_{\mathrm{Z}}$ will be well covered at hadron colliders, while (as we will see) the intermediate mass region is not easy for hadron colliders, unless a super-high-luminosity interaction region which focuses on electromagnetic triggers is available [22], the need for data on the Higgs boson spectrum provides a strong scientific justification for building such an $\mathrm{e}^{+} \mathrm{e}^{-}$ collider. However, the time scale for the construction of an $\mathrm{e}^{+} \mathrm{e}^{-}$collider beyond SLC or LEP is very uncertain. Indeed, a high-energy, high-luminosity hadron collider is the most likely near-future facility, so we will concentrate in the following on means for detecting an intermediate mass Higgs boson at such a machine. As suggested in earlier studies [18], the best hope may lie in the rare decay modes of the $\mathrm{H}^{0}$ and $\mathrm{H}^{ \pm}$. We will present a detailed analysis of the rates for all such modes, and evaluate the machine and detector characteristics that are needed to eventually obtain complete information about the scalar spectrum from a hadron collider.

At the present time the $\mathrm{t}$-quark mass is unknown. Our results will depend upon $m_{\mathrm{t}}$ in three ways. First, if $\mathrm{H}^{0} \rightarrow \mathrm{t}_{\mathrm{t}}$ is allowed, or $\mathrm{H}^{+} \rightarrow \overline{\mathrm{tb}}$ is allowed, then the branching ratios for other decays are smaller; generally it is easier to detect $\mathbf{H}^{0}, \mathrm{H}^{ \pm}$ if $m_{\mathrm{H}^{0}}<2 m_{\mathrm{t}}, m_{\mathrm{H}^{ \pm}}<m_{\mathrm{t}}+m_{\mathrm{b}}$. Second, many of the decays involving photons occur at one loop, and rates depend upon $m_{\mathrm{t}}$ through the loops containing the $\mathrm{t}$ quark. Typically this dependence is not very strong since gauge boson loops dominate fermion loops, but it must be included. Third, the production cross sections vary significantly with $m_{t}$, especially in the intermediate mass range. Consequently, we will present results for the $\mathrm{H}^{0}$ in several plots in $m_{\mathrm{t}}-m_{\mathrm{H}^{0}}$ parameter space, showing for each decay mode the boundary between the regions where it is, and is not, detectable. If $m_{\mathrm{t}} \leqq 125 \mathrm{GeV}$ the top will surely be discovered at the Tevatron collider; larger values of $m_{\mathrm{t}}$ will be accessible at larger luminosity. After the $\mathrm{t}$ is discovered our graphs can be used by looking at fixed $m_{\mathrm{t}}$. For the $\mathrm{H}^{ \pm}$we will simply outline the large variety of possibilities.

We can summarize the main implications of our study for detector design.

(i) It is very important to detect isolated photons and leptons with energy above about $30 \mathrm{GeV}$, and to distinguish them from jets at a level of better than $10^{-4}$.

(ii) Since any intermediate mass Higgs boson will have a width small compared to a few $\mathrm{GeV}$, the background to its detection will decrease linearly with mass resolution. Improving resolution for the final states $\gamma \gamma, \gamma \ell^{+} \ell^{-}, \ell \nu \gamma, \tau^{+} \tau^{-}, \ldots$ will be very valuable. 
Concerning machine parameters, two remarks are in order. Since most searches involve rare modes, luminosity is very important. As we will see, an intermediate mass $\mathrm{H}^{0}$ is probably detectable at the SSC if full luminosity and design energy are available. However, its detection would be greatly facilitated if $\mathscr{L}>10^{33} \mathrm{~cm}^{-2} \mathrm{sec}^{-1}$ were possible. Even though the $\mathrm{H}^{0}$ 's we consider are light, the energy of the collider is important; the cross section for intermediate mass $\mathrm{H}^{0}$ production is four-five times larger at the SSC than at $\sqrt{s}=17 \mathrm{TeV}$ (at $m_{\mathrm{t}}=40 \mathrm{GeV}$ ), directly reflecting the larger gluon luminosity at higher energies. This results in substantially larger statistical significance for a typical rare decay mode, especially since many of the background processes grow more slowly.

In the following we will consider neutral scalar Higgs bosons which occur in both the $\mathrm{SM}$ and in supersymmetry, and then $\mathrm{H}^{+}$, which occurs in all extensions of the SM. First branching ratios will be given and discussed. Then, mode by mode we will estimate the signal-to-background (S/B) situation for various machine parameters and detector resolutions. In addition we will assess the impact of new physics, such

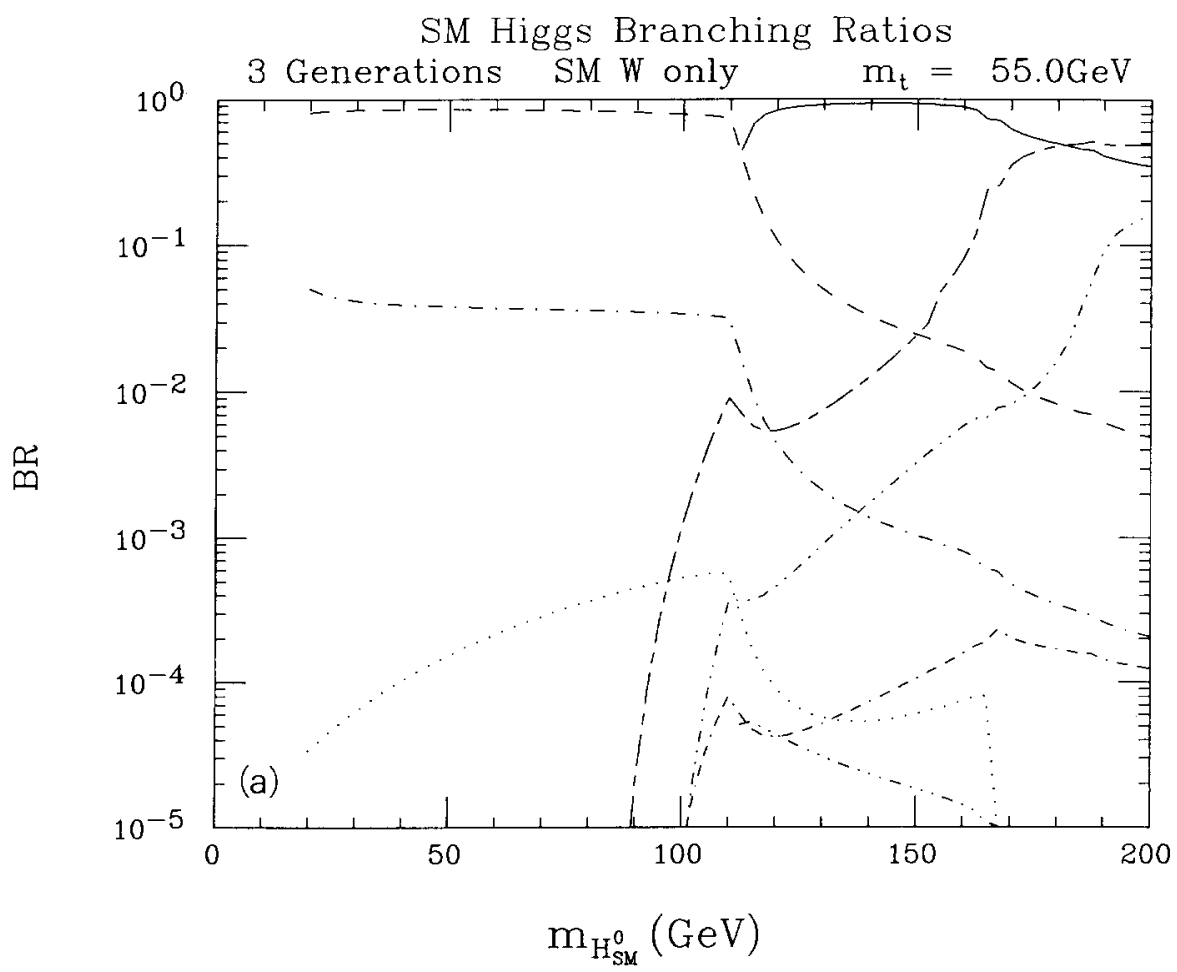

Fig. 1. We plot branching ratios for all the $\mathrm{H}_{\mathrm{SM}}^{0}$ decay modes of interest, for $m_{\mathrm{t}}=55 \mathrm{GeV}$ and $m_{\mathrm{t}}=90$ $\mathrm{GeV}$. The curves for the various channels are: solid $=\overline{\mathrm{t}}$; dashes $=\mathrm{b} \overline{\mathrm{b}}$; dashdot $=\tau^{+} \tau^{-}$; longdash-shortdash $=W W$ or $W^{*}$ (with no $W, W^{*}$ branching ratios included); dash-doubledot $=Z^{*}$ (no $Z, Z^{*}$ branching ratios included); dots $=\gamma \gamma$; doubledash-dot $=\mathrm{Z} \gamma ;$ and dash-tripledot $=\Theta \gamma$. 


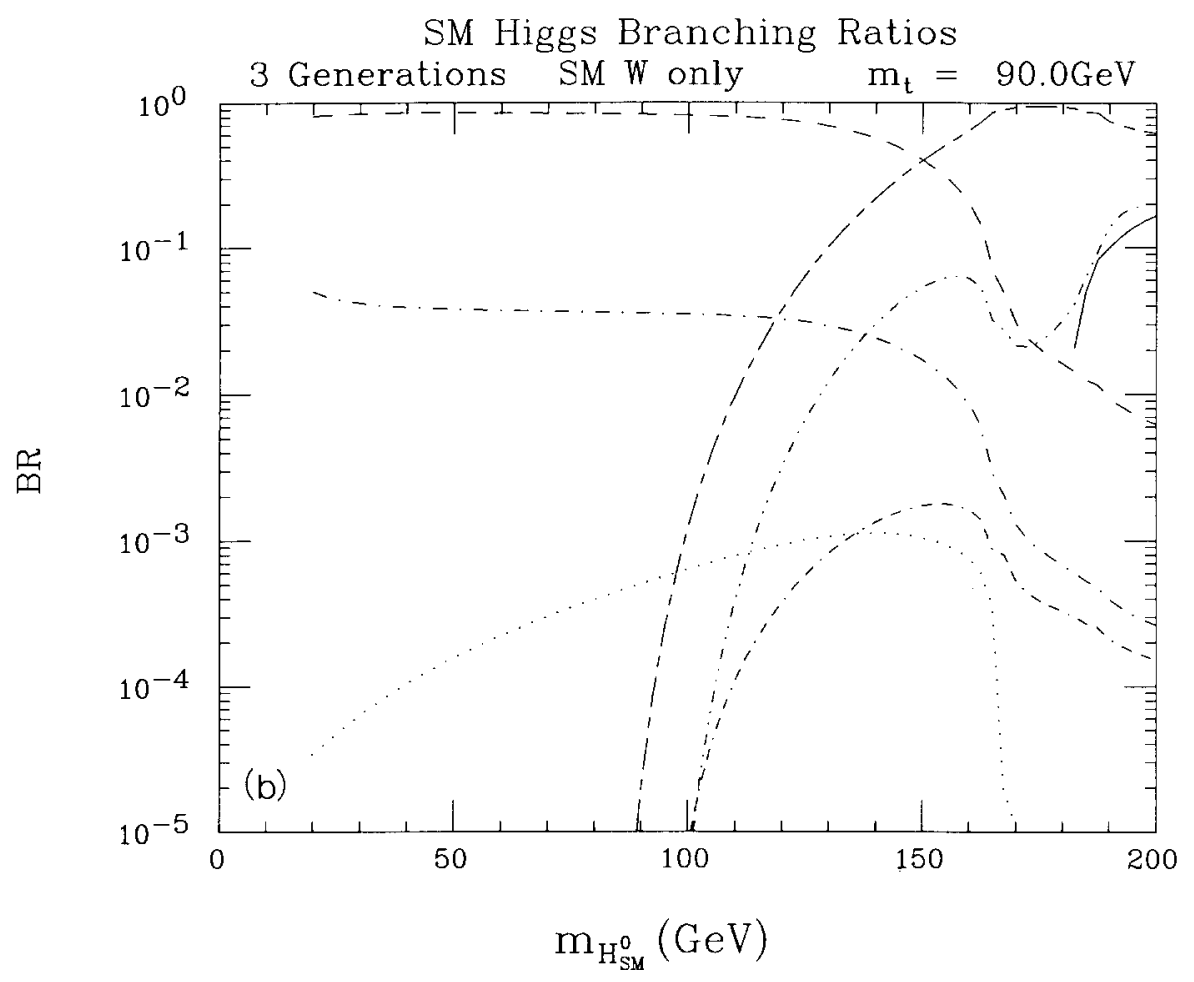

Fig. 1 (continued).

as supersymmetry, additional gauge bosons, or an additional family, upon the rare modes controlled by loop diagrams. A few examples will be given. In particular, we emphasize that if intermediate mass Higgs scalars exist, measurement of their rare branching ratios will determine a variety of Higgs couplings and will provide a powerful indicator of the presence or absence of new physics in the controlling loop diagrams. Although we will emphasize standard model calculations, with some remarks about supersymmetric theories and other possibilities, the reader should keep in mind that the "rare" branching ratios could get quite large in some approaches such as those with composite Higgs bosons [23].

\section{Neutral Higgs boson(s)}

\subsection{BRANCHING RATIOS}

In fig. 1 we present the branching ratios for a $\mathrm{SM} \mathrm{H}_{\mathrm{SM}}^{0}$ decaying to $\overline{\mathrm{t}}, \mathrm{b} \overline{\mathrm{b}}, \tau^{+} \tau^{-}$, $\gamma \gamma, \Theta \gamma, \mathrm{Z} \gamma, \mathrm{ZZ}^{*}, \mathrm{~W}^{ \pm} \mathrm{W}^{\mp *}, \mathrm{ZZ}$, and $\mathrm{W}^{+} \mathrm{W}^{-}$. Figs. $1 \mathrm{a}$ and $1 \mathrm{~b}$ are for $m_{\mathrm{t}}=55 \mathrm{GeV}$ and $90 \mathrm{GeV}$, respectively. Results for $m_{\mathrm{t}}>90 \mathrm{GeV}$ are very similar to those at $m_{\mathrm{t}}=90 \mathrm{GeV}$. 
For the reader's convenience we will summarize the formulae for the various modes. The fermion, $\mathrm{W}^{+} \mathrm{W}^{-}$, and $\mathrm{ZZ}$ modes are standard, with widths

$$
\Gamma\left(\mathrm{H}_{\mathrm{SM}}^{0} \rightarrow \mathrm{ff}\right)=\frac{\kappa G_{\mathrm{F}} m_{\mathrm{f}}^{2} m_{\mathrm{H}_{\mathrm{SM}}^{0}}}{4 \pi \sqrt{2}}\left(1-\frac{4 m_{\mathrm{f}}^{2}}{m_{\mathrm{H}_{\mathrm{SM}}^{0}}^{2}}\right)^{3 / 2},
$$

where $\kappa=1$ or 3 for leptons or quarks, and

$$
\begin{gathered}
\Gamma\left(\mathrm{H}_{\mathrm{SM}}^{0} \rightarrow \mathrm{W}^{+} \mathrm{W}^{-}\right)=\frac{G_{\mathrm{F}} m_{\mathrm{H}_{\mathrm{SM}}^{0}}^{3}}{8 \pi \sqrt{2}}\left(1-\frac{4 m_{\mathrm{W}}^{2}}{m_{\mathrm{H}_{\mathrm{SM}}^{0}}^{2}}\right)^{1 / 2}\left(1-\frac{4 m_{\mathrm{W}}^{2}}{m_{\mathrm{H}_{\mathrm{SM}}^{0}}^{2}}+\frac{12 m_{\mathrm{W}}^{4}}{m_{\mathrm{H}}^{4}}\right), \\
\Gamma\left(\mathrm{H}_{\mathrm{SM}}^{0} \rightarrow \mathrm{ZZ}\right)=\frac{G_{\mathrm{F}} m_{\mathrm{H}_{\mathrm{SM}}^{0}}^{3}}{16 \pi \sqrt{2}}\left(1-\frac{4 m_{\mathrm{Z}}^{2}}{m_{\mathrm{H}_{\mathrm{SM}}^{0}}^{2}}\right)^{1 / 2}\left(1-\frac{4 m_{\mathrm{Z}}^{2}}{m_{\mathrm{H}_{\mathrm{SM}}^{0}}^{2}}+\frac{12 m_{\mathrm{Z}}^{4}}{m_{\mathrm{H}_{\mathrm{SM}}^{0}}^{4}}\right) .
\end{gathered}
$$

For completeness, we give some of the corresponding results for the two-Higgs doublet minimal supersymmetric theory [24,25]. The lighter neutral Higgs boson, h, of such a model has ff widths related to those of a SM Higgs of the same mass by

$$
\begin{array}{r}
\Gamma\left(\mathrm{h} \rightarrow \mathrm{b} \overline{\mathrm{b}} \text { or } \tau^{+} \tau^{-}\right)=\frac{\sin ^{2} \alpha}{\cos ^{2} \beta} \Gamma\left(\mathrm{H}_{\mathrm{SM}}^{0} \rightarrow \mathrm{f} \overline{\mathrm{f}}\right), \\
\Gamma(\mathrm{h} \rightarrow \overline{\mathrm{t}} \text { or } c \overline{\mathrm{c}})=\frac{\cos ^{2} \alpha}{\sin ^{2} \beta} \Gamma\left(\mathrm{H}_{\mathrm{SM}}^{0} \rightarrow \mathrm{f} \overline{\mathrm{f}}\right),
\end{array}
$$

where $\tan \beta=v_{2} / v_{1}$ and $\alpha$ is a mixing angle arising from constructing the Higgs mass eigenstates; $\alpha$ depends on the parameters of the Higgs potential (see refs. [24] and [25]), and $v_{1}$ and $v_{2}$ are the vacuum expectation values of the neutral fields of the original two complex doublets. (The SM limit for $\mathrm{h}$ is given by $-\alpha=\beta=\frac{1}{4} \pi$.) It should also be noted that the light $h$ of the minimal supersymmetry model has mass $m_{\mathrm{h}} \leqslant m_{\mathrm{Z}}$ and is too light to decay to WW or ZZ. The pseudoscalar Higgs of a two-doublet model, A, may have any mass (but in the minimal SUSY model its mass is determined once those of the Higgs scalars are specified). It has no coupling to $\mathrm{WW}$ or $\mathrm{ZZ}$ and has ff widths given by

$$
\begin{gathered}
\Gamma\left(\mathrm{A} \rightarrow \mathrm{b} \overline{\mathrm{b}} \text { or } \tau^{+} \tau^{-}\right)=\frac{\kappa G_{\mathrm{F}} m_{\mathrm{f}}^{2} m_{\mathrm{A}} \tan ^{2} \beta}{4 \pi \sqrt{2}}\left(1-\frac{4 m_{\mathrm{f}}^{2}}{m_{\mathrm{A}}^{2}}\right)^{1 / 2}, \\
\Gamma(\mathrm{A} \rightarrow \mathrm{t} \bar{t} \text { or } \mathrm{c} \overline{\mathrm{c}})=\frac{3 G_{\mathrm{F}} m_{\mathrm{f}}^{2} m_{\mathrm{A}} \cot ^{2} \beta}{4 \pi \sqrt{2}}\left(1-\frac{4 m_{\mathrm{f}}^{2}}{m_{\mathrm{A}}^{2}}\right)^{1 / 2} .
\end{gathered}
$$

Note the different power of $\left(1-4 m_{\mathrm{f}}^{2} / m_{\mathrm{A}}^{2}\right)$ in eqs. (6), (7) compared to the analo- 

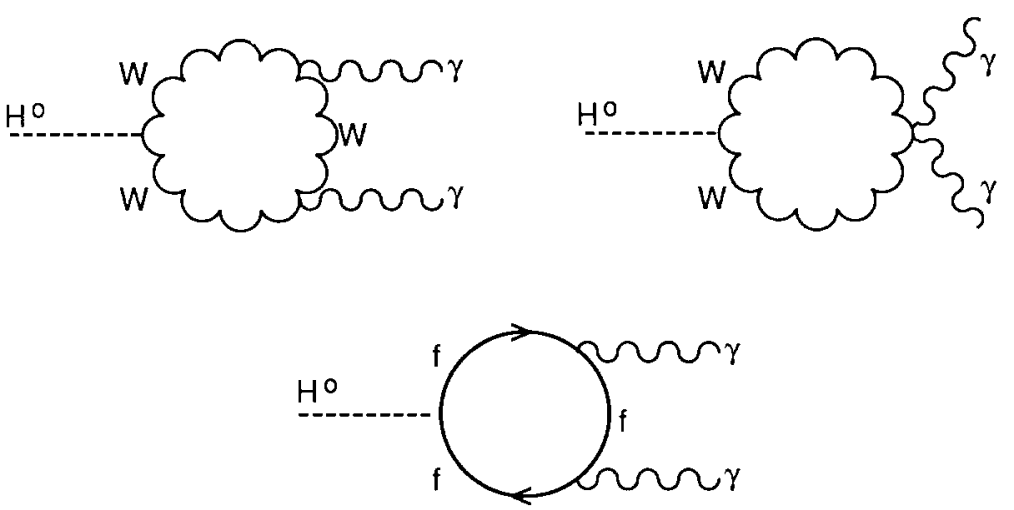

Fig. 2. Feynman diagrams for $\mathrm{H}^{0} \rightarrow \gamma \gamma$, in the standard model. The third graph represents a generic fermion loop.

gous threshold behavior in eq. (1). If branching ratios to several different fermion channels are measureable it will allow [26] an experimental determination of the parity of any spin zero bosons that are discovered. The second, heavy, neutral scalar Higgs boson, $\mathrm{H}$, in the minimal SUSY model has mass $m_{\mathrm{H}} \geqslant m_{\mathrm{Z}}$ and widths to fermion anti-fermion pairs which exhibit $[24,25]$ different dependence on $\alpha$ and $\beta$ than that given in eqs. (4) and (5). It is also very weakly coupled to the WW and ZZ decay channels if its mass is larger than $2 m_{\mathrm{W}}$. (In this limit, minimal SUSY predicts that the allowed WW and ZZ coupling to the neutral Higgs bosons is saturated by the light scalar, h.) Consequently, it could turn out to be necessary to search for this heavier scalar Higgs of supersymmetry in the same rare decay modes that we discuss for the SM intermediate mass Higgs and for the lighter scalar and the pseudoscalar of supersymmetry. We now turn to a consideration of the rare decay modes not already encompassed by the above formulae. The explicit formulae given assume standard model couplings to the particles involved; generalizations will be briefly discussed later.

2.1.1. $H^{0} \rightarrow \gamma \gamma$. This decay can occur with scalar, fermion, and gauge boson loops as shown in fig. 2. It was originally calculated [27] in the limit of heavy gauge bosons, $m_{\mathrm{H}^{0}} \ll m_{\mathrm{W}}$. Later, the general result below was obtained [28]:

$$
\Gamma\left(\mathrm{H}^{0} \rightarrow \gamma \gamma\right)=\frac{\alpha^{2} G_{\mathrm{F}} m_{\mathrm{H}^{0}}^{3}}{64 \sqrt{2} \pi^{3}}\left|\sum_{i} F_{i} Q_{i}^{2}\right|^{2},
$$

where $i=$ scalar, fermion, gauge boson, $Q_{i}$ is its electric charge in units of $e$, and

$$
\begin{aligned}
F_{\text {scalar }} & =\tau\left(1-\tau I^{2}\right), \\
F_{\text {fermion }} & =-2 \tau\left[1+(1-\tau) I^{2}\right], \\
F_{\text {gauge boson }} & =2+3 \tau+3 \tau(2-\tau) I^{2},
\end{aligned}
$$


with

$$
\begin{aligned}
\tau=\frac{4 m_{i}^{2}}{m_{\mathrm{H}^{0}}^{2}}, & \begin{cases}\tan ^{-1} \frac{1}{\sqrt{\tau-1}}, & \tau>1 \\
\frac{1}{2}\left[\pi+i \ln \frac{1+\sqrt{1-\tau}}{1-\sqrt{1-\tau}}\right], & \tau<1 .\end{cases}
\end{aligned}
$$

A factor of 3 must be included for a colored loop in the appropriate $F$. All the threshold behavior necessary to consider $m_{\mathrm{H}^{0}}$ in the same region as $2 m_{\mathrm{t}}$ and $2 m_{\mathrm{W}}$ is included here. The above formulas must be modified to account for any non-SM couplings of the $\mathrm{H}^{0}$ or the $\gamma$ to the scalars, fermions, or gauge bosons in any given

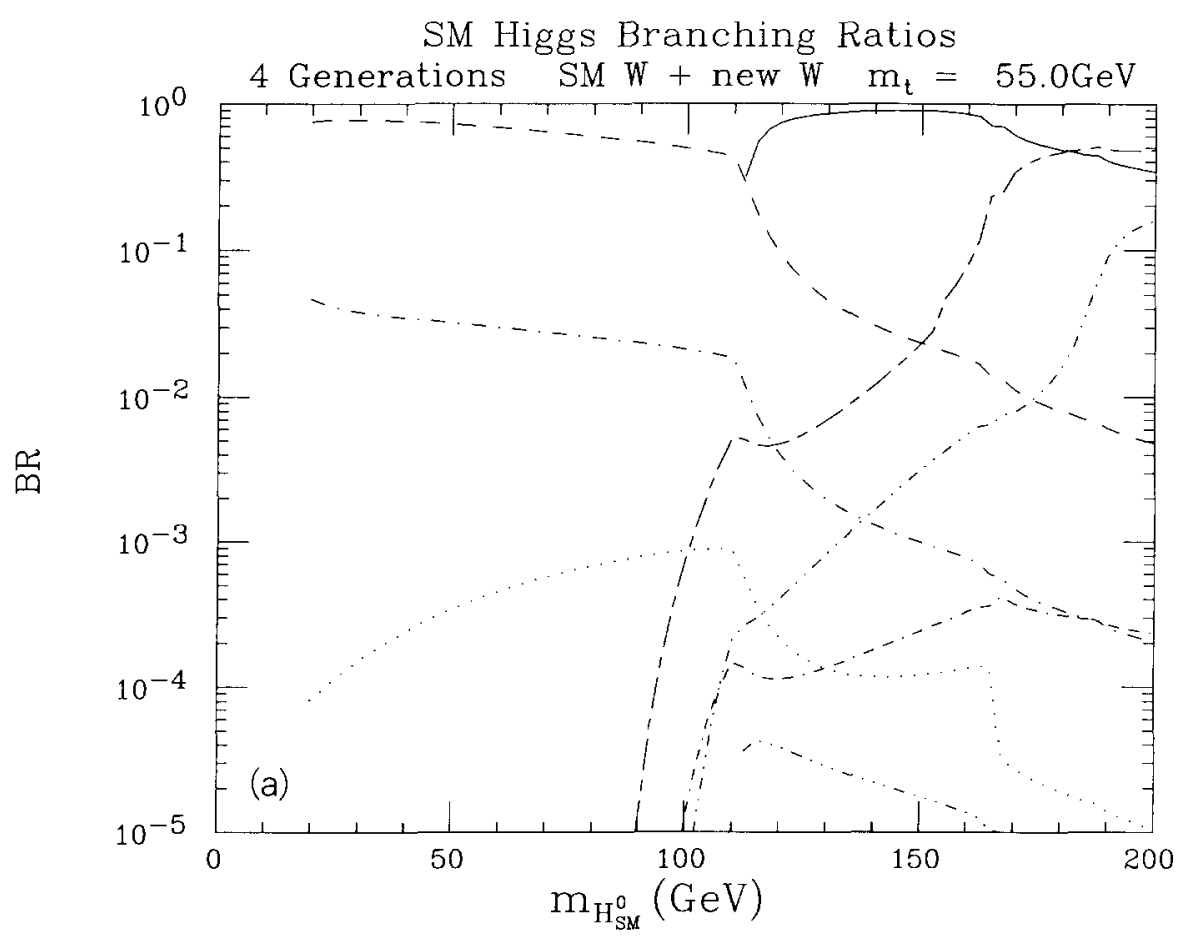

Fig. 3. We plot the branching ratios for $\mathrm{H}_{\mathrm{SM}}^{0}$ decays in the manner of fig. 1 , at $m_{\mathrm{t}}=55 \mathrm{GeV}$, but including three different possible new loop contributions beyond the standard model. In fig. 3a we include a fourth generation with a heavy lepton mass of $100 \mathrm{GeV}$, a new down quark with mass $300 \mathrm{GeV}$, and a new up quark with mass $300 \mathrm{GeV}$. In fig. 3b we include a new charged $\mathrm{W}$ of mass $1 \mathrm{TeV}$, but no fourth generation. Finally, in fig. $3 \mathrm{c}$ we include both the above new $\mathrm{W}$ and fourth generation. 


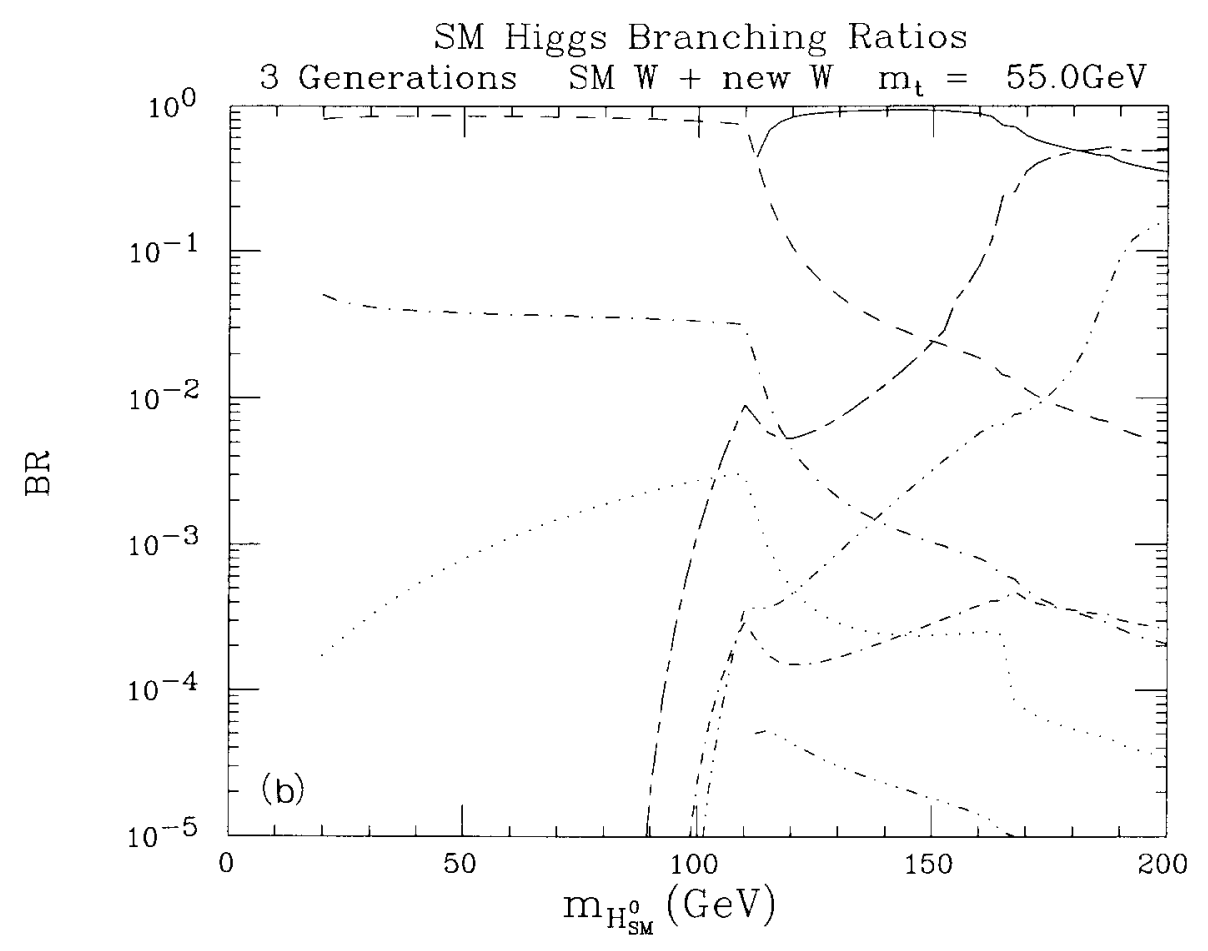

Fig. 3 (continued).

loop. Some consideration has been given [28] to the question of gluon corrections to the quark loops; it was argued that they are small except near the q $\bar{q}$ threshold. Since $\mathrm{W}$ loops dominate for our situation, we do not consider these corrections further, but they should be kept in mind if extra generations of heavy fermions are considered. We have given results in the intermediate mass range for the SM $\mathrm{H}_{\mathrm{SM}}^{0} \rightarrow \gamma \gamma$ in fig. 1 , when only 3 families and the standard $\mathrm{W}$ are present. In fig. 3 we give examples at $m_{\mathrm{t}}=55 \mathrm{GeV}$ of the effect of a new heavy family or new W gauge boson or both; in each case SM coupling is assumed. Obviously the $\gamma \gamma$ width and branching ratio is a sensitive measure of new physics. In supersymmetric models, the various different supersymmetric partners must be included [29] in the loop amplitudes. In addition, as mentioned earlier the normal fermions and gauge bosons have altered couplings to the $\mathrm{h}$ and $\mathrm{H}$ which must be incorporated.

2.1.2. $H^{o} \rightarrow Z \gamma$. The computations for this decay are similar to those for the $\gamma \gamma$ mode. The results in terms of parametric integrals are given in ref. [30]. In appendix A, we give analytic results for all the different loop contributions, again for the SM $\mathrm{H}_{\mathrm{SM}}^{0}$. Altered couplings would have to be incorporated in typical supersymmetry models. The branching ratios for this decay are given in figs. 1 and 3 for the same cases as considered for the $\gamma \gamma$ mode. Even though the $Z \gamma$ and $\gamma \gamma$ branching ratios 


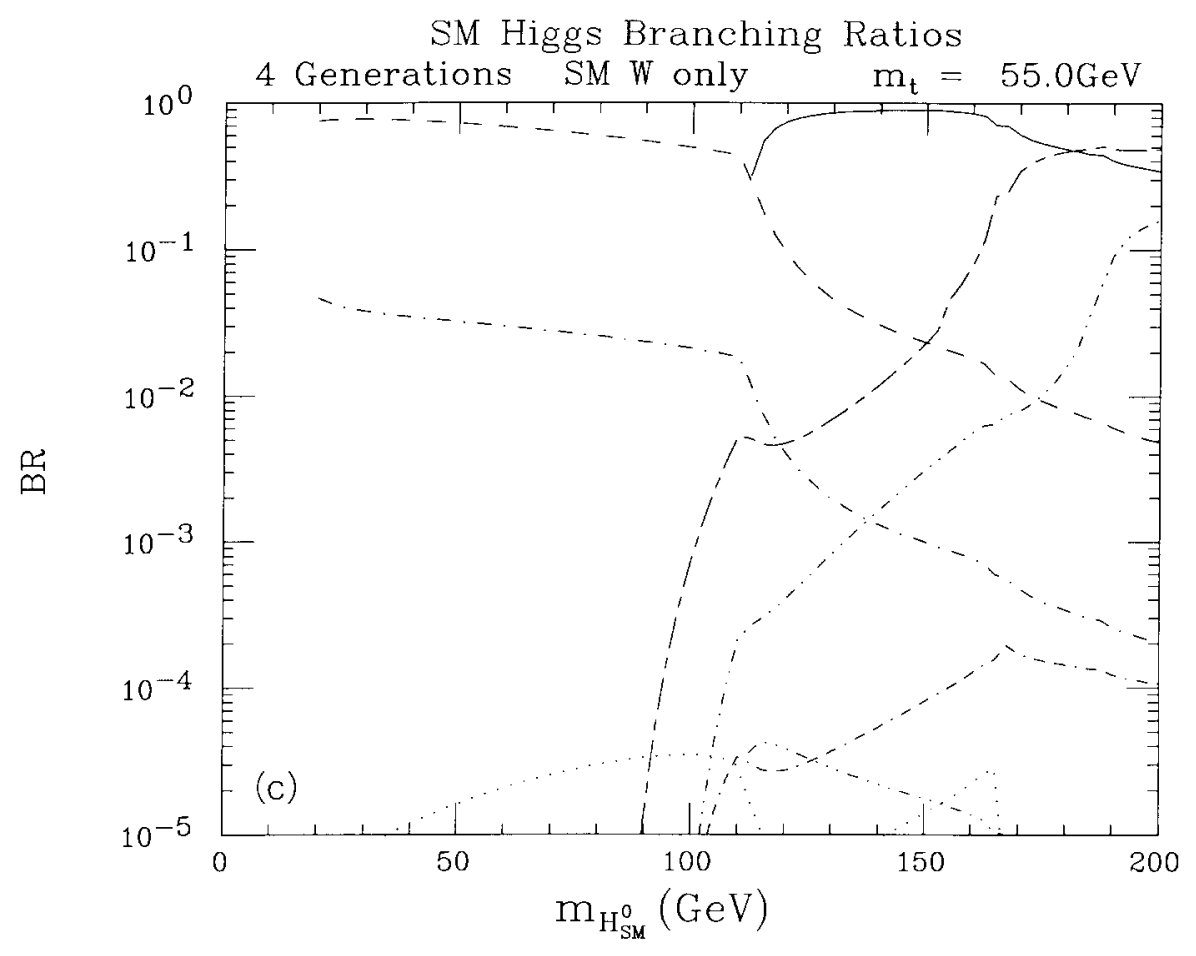

Fig. 3 (continued).

are generally comparable, the $Z \gamma$ mass cannot be determined with excellent resolution (required to eliminate backgrounds) except when the $\mathrm{Z}$ decays to $\ell^{+} \ell^{-}$. The associated branching ratio of 0.06 (summing over $\mu^{+} \mu^{-}$and $\mathrm{e}^{+} \mathrm{e}^{-}$) generally results in fewer useable events than are found at the same mass in the $\gamma \gamma$ mode.

2.1.3. $H^{0} \rightarrow \Theta \gamma$. Estimating the rate for $\mathrm{H}^{0} \rightarrow \Theta \gamma$, where $\Theta$ is the toponium ${ }^{3} \mathrm{~S}_{1}$ state, requires some care. For $m_{\Theta}<m_{\mathrm{H}^{0}}<2 m_{\mathrm{Z}}$ the problem is essentially a nonrelativistic one for $\mathrm{H}^{0}$ and $\Theta$, so that the simplest bound state arguments should apply. The $t$ and $\bar{t}$ are produced essentially at rest and can easily bind. In contrast, for $\mathrm{H}^{0} \rightarrow \Upsilon_{\gamma}$ or $\mathrm{H}^{0} \rightarrow \Psi \gamma$ the $\bar{b} \bar{b}$ or cc quarks would be moving apart with a large relative velocity and would be less likely to bind to $\Upsilon$ or $\Psi$. In such a case an alternative approach relying on the perturbative formalism of ref. [31] might be appropriate. Remarkably one obtains exactly the same answer as in the non-relativistic formalism.

In either approach one obtains (using standard model couplings)

$$
\Gamma\left(\mathrm{H}^{0} \rightarrow \Theta \gamma\right)=\frac{3 G_{\mathrm{F}} m_{\mathrm{H}^{0}}^{2}}{4 \pi \sqrt{2} \alpha}\left(1-\frac{m_{\Theta}^{2}}{m_{\mathrm{H}^{0}}^{2}}\right) \Gamma\left(\Theta \rightarrow \mu^{+} \mu^{-}\right) .
$$


There is a factor of 3 here, relative to the crossed process $\Theta \rightarrow \mathrm{H}^{0} \gamma$ because of spin summing versus spin averaging for the $\Theta$. Radiative corrections are large in the reversed decay $\Theta \rightarrow \gamma \mathrm{H}^{0}$ [32] and could also be large here. However, we have not computed them and will use the lowest order result above. We will assume that

$$
\Gamma\left(\Theta \rightarrow \mu^{+} \mu^{-}\right) \approx \Gamma\left(\Psi \rightarrow \mu^{+} \mu^{-}\right)=4.7 \mathrm{keV}
$$

since the leptonic widths of the heavy quarkonium states scale approximately as $e_{\mathrm{q}}^{2}$. This approximate value implies

$$
\frac{\Gamma\left(\mathrm{H}^{0} \rightarrow \Theta \gamma\right)}{\Gamma\left(\mathrm{H}^{0} \rightarrow \gamma \gamma\right)}=\frac{5733\left(1-m_{\Theta}^{2} / m_{\mathrm{H}^{0}}^{2}\right)}{m_{\mathrm{H}^{0}}(\mathrm{GeV})\left|\sum F_{i} Q_{i}^{2}\right|^{2}}
$$

Here the $F_{i}$ 's are from eq. (9). For a large range of masses this ratio is in the range 0.1 to 1 . The branching ratio for this decay appears, when allowed, in figs. 1 and 3 . The detectability of this mode is, however, limited by the fraction of the $\Theta$ decays that can be separated from background. In the mass range of interest, about $3 \%$ of $\Theta$ decays are to $\mathrm{e}^{+} \mathrm{e}^{-}$and $\mu^{+} \mu^{-}$(each), so that $10 \%$ is probably a good estimate of useable $\Theta$ decays. This indicates that $10^{-5}$ to $10^{-4}$ of $\mathrm{H}_{\mathrm{SM}}^{0}$ decays can be detected with $\mathrm{H}_{\mathrm{SM}}^{0} \rightarrow \Theta \gamma$ followed by $\Theta \rightarrow \ell^{+} \ell^{-}$or any other useable modes. Consequently, as we shall later see, the $\mathrm{H}_{\mathrm{SM}}^{0}$ decay in these modes may be detectable as a confirmation of discovery in the $\gamma \gamma$ mode and other modes. If so we will be able to obtain a measurement of the $\mathrm{H}_{\mathrm{SM}}^{0}{ }^{\mathrm{t} t}$ coupling.

2.1.4. $H^{0} \rightarrow Z Z^{*}$ followed by $Z^{*} \rightarrow f \bar{f}$. The tree level diagram for this mode is lower order in perturbation theory than other (loop) diagrams contributing to the same final state (e.g. $\mathrm{H}^{0} \rightarrow \mathrm{Z} \gamma^{*}$ followed by $\gamma^{*} \rightarrow \mathrm{ff}$ ), and neglect of interference should be a good approximation when $m_{\mathbf{H}^{0}}$ approaches the $\mathrm{ZZ}$ threshold. The tree level results for this branching ratio (from ref. [33]) are given in figs. 1 and 3 . However, in order to achieve the mass resolution required to eliminate backgrounds, it is necessary to employ $\ell^{+} \ell^{-}$channels for both the $Z$ and $Z^{*}$ decay channels: The resulting event rate is generally larger than that for the $Z \gamma$ mode, while backgrounds are comparable. As a result, the $\mathrm{H}_{\mathrm{SM}}^{0}$ is likely to be detectable in this mode if $m_{\mathrm{H}_{\mathrm{SM}}^{0}}$ is near $m_{Z}$, the precise boundary in $m_{\mathrm{H}_{\mathrm{SM}}^{0}}$ for detectability depending upon $m_{\mathrm{t}}$.

Of course, this decay channel will be of much less use in the case of the minimal SUSY model heavy scalar, $H$, since its $Z Z$ couplings are somewhat suppressed in the region $m_{\mathrm{H}} \leq 2 m_{\mathrm{Z}}$ where the $\mathrm{ZZ} Z^{*}$ mode is of potential interest.

\subsection{HIGGS TOTAL WIDTH}

Having computed the partial widths to all channels, including the gg decay channel as well as those explicitly displayed in fig. 1, a summation over all decay modes yields the Higgs total width. This is plotted in the case of the $\mathrm{SM} \mathrm{H}_{\mathrm{SM}}^{0}$ in fig. 4. The width is dominated by the $\mathrm{b} \overline{\mathrm{b}}, \overline{\mathrm{t}}, \tau^{+} \tau^{-}$and $\mathrm{gg}$ channels, until $m_{\mathrm{H}_{\mathrm{SM}}^{\mathrm{o}}}$ 


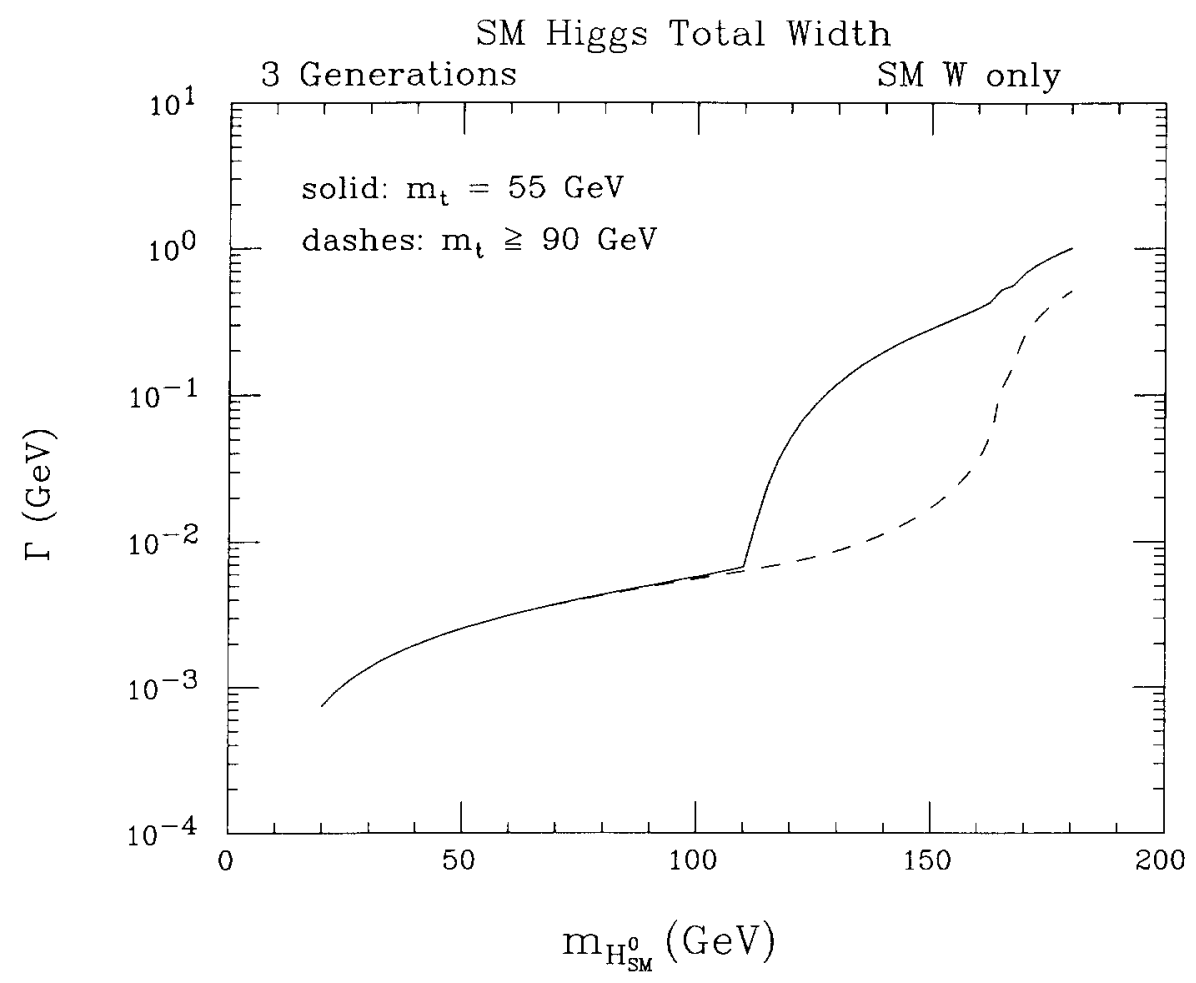

Fig. 4. The total width $\Gamma_{\mathrm{H}_{\mathrm{SM}}^{0}}$ for the SM Higgs, after summing over all channels, for two different $m_{\mathrm{t}}$ values. For $m_{\mathrm{t}}$ values still higher than the $90 \mathrm{GeV}$ case there is little change.

approaches the WW decay threshold. The most important point to note is that, independent of $m_{t}$, the width is always less than $1 \%$ of the Higgs mass in the intermediate mass range being considered. Thus, accepting events in a mass bin of size $2 \% m_{\mathrm{H}^{0}}$, as we shall do below, will leave intact almost the entire contribution of the Higgs boson decay to that channel.

\subsection{HIGGS PRODUCTION CROSS SECTIONS}

Over the mass region of interest, the principle contributing production process is gg fusion via triangle diagrams. One of the largest contributing diagrams is that containing the top quark, and hence there is some sensitivity of the cross section to $m_{\mathrm{t}}$. The contribution from WW fusion is not sensitive to $m_{\mathrm{t}}$ but is smaller than that from gg fusion. Finally, the $\mathrm{t}_{\mathrm{t}}$ fusion process is in general present; however, it is even smaller than the previous two contributions. A survey of these various contributions was carried out in ref. [34], including dependence on the top mass. For the reader's convenience we give the results for $g g$ fusion as a function of the Higgs mass $m_{\mathrm{H}^{0}}$, for a variety of $m_{\mathrm{t}}$ values, in fig. 5. Standard model couplings are 


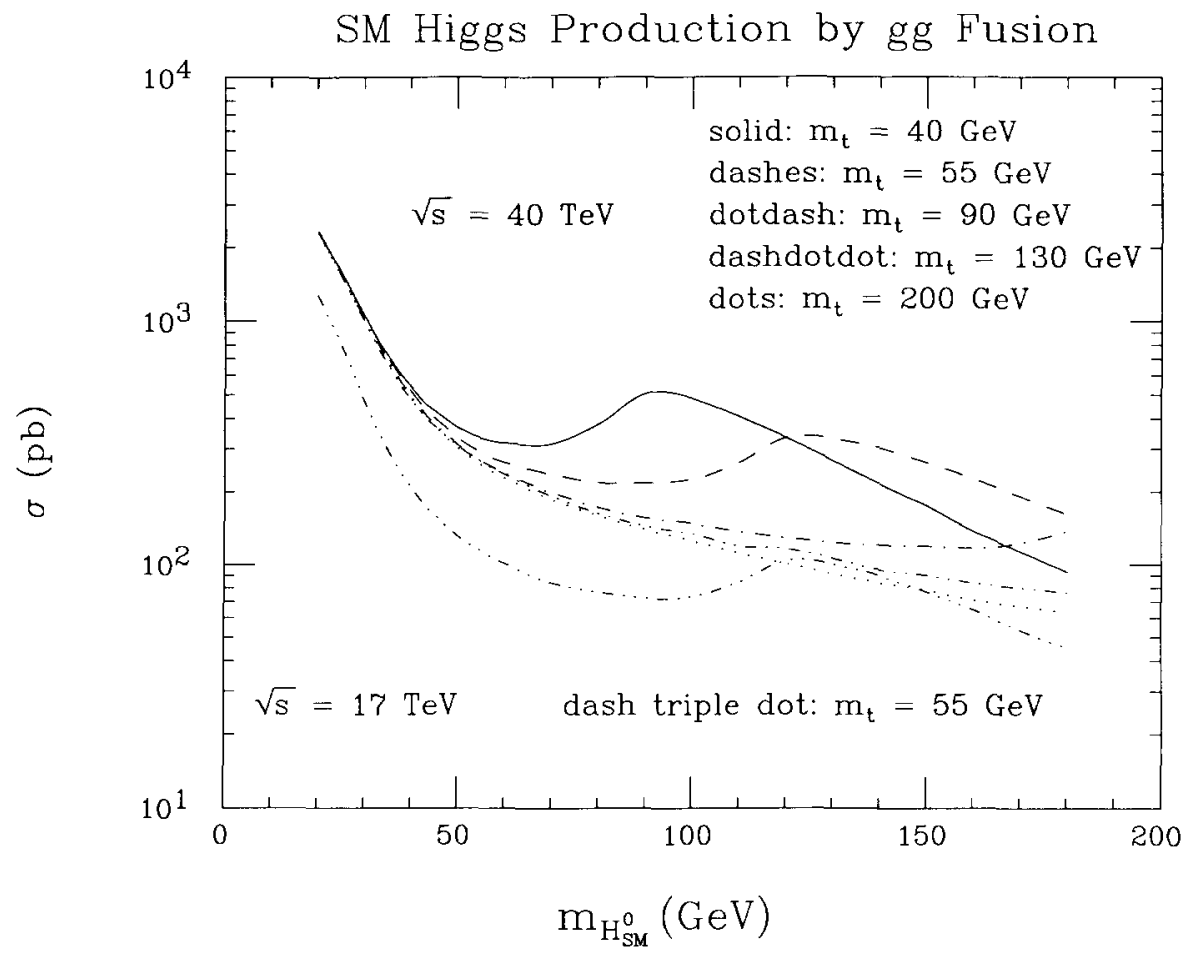

Fig. 5. Cross sections for gg fusion as a function of $m_{\mathrm{H}^{0}}$ in the intermediate mass range, for a variety of $m_{\mathrm{t}}$ values. Standard model couplings are assumed. In this figure, but not in ref. [34] we have used the same $g$ distribution function at every $m_{\mathrm{t}}$ value (namely that for $m_{\mathrm{t}}=40 \mathrm{GeV}$ ). However, the variation of the $g$ distribution function with $m_{\mathrm{t}}$ in the region of interest is small. One curve at $m_{\mathrm{t}}=55 \mathrm{GeV}$ is given for $\sqrt{s}=17 \mathrm{TeV}$, in order to compare with the corresponding $\sqrt{s}=40 \mathrm{TeV}$ curve.

assumed. Note that cross sections at $\sqrt{s}=17 \mathrm{TeV}$ are substantially below those at $\sqrt{s}=40 \mathrm{TeV}$. This is so, even at intermediate $m_{\mathrm{H}^{0}}$, since as $\sqrt{s}$ increases for fixed $m_{\mathrm{H}^{0}}$ the gluon structure functions are evaluated at smaller $x$, where they are larger. At SSC energies we note that the cross section for Higgs production is generally in the range of 200 to $300 \mathrm{pb}$ at $m_{\mathrm{t}}=55 \mathrm{GeV}$, and about half that at $m_{\mathrm{t}}=90 \mathrm{GeV}$. This implies that, for the standard SSC luminosity of

$$
L_{\text {year }}=10^{4} \mathrm{pb}^{-1} \text {, }
$$

there will be several times $10^{6}$ events per year. Thus, despite the relatively small branching ratios found in section $\mathrm{A}$, there will be hundreds, or, for some cases in the $\gamma \gamma$ mode, thousands of events in a typical high mass resolution channel involving only final state leptons and/or photons. To know if these event rates are useful requires an investigation of the backgrounds in each of these channels. 


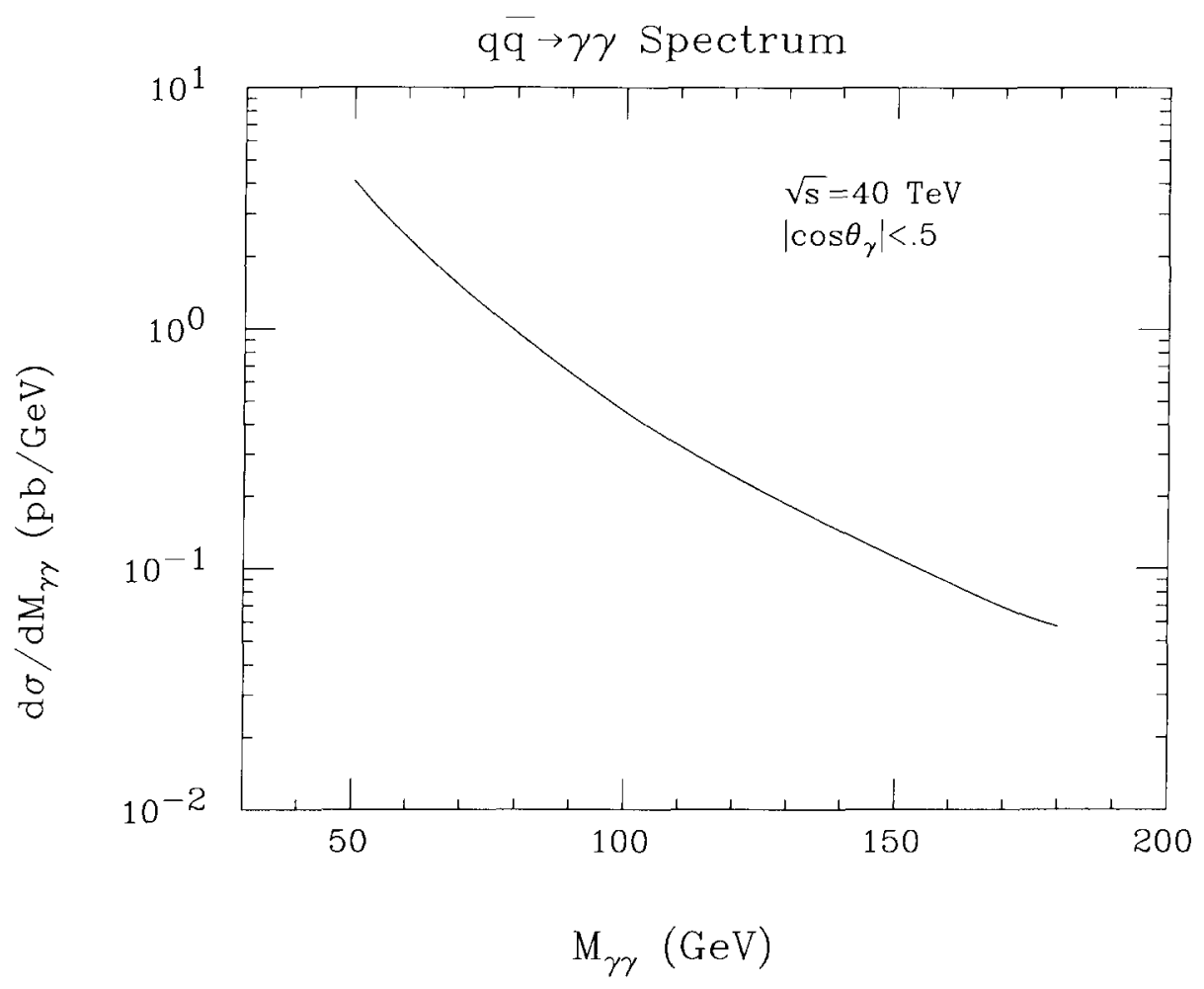

Fig. 6. The $\gamma \gamma$ spectrum $\mathrm{d} \sigma / \mathrm{d} M_{\gamma \gamma}$ at $\sqrt{s}=40 \mathrm{TeV}$ coming from $\mathrm{q} \overline{\mathrm{q}} \rightarrow \gamma \gamma$. A cut of $\left|\cos \theta_{\gamma}\right|<0.5$ has been imposed on the angles of the outgoing $\gamma$ 's in their center of mass.

\subsection{BACKGROUNDS}

2.4.1. $H^{0} \rightarrow \gamma \gamma$. The most obvious background to the $\gamma \gamma$ mode is from $\mathrm{q} \overline{\mathrm{q}} \rightarrow \gamma \gamma$. The calculation is straightforward and has been done a number of times. (See, for example, ref. [18].) The main uncertainty is due to the effects of structure functions. Some of this uncertainty cancels in the ratio of signal to background, but not all since the signal depends mainly on the gluon structure functions, while the background depends mainly on the quark and antiquark structure functions. A plot of the spectrum $\mathrm{d} \sigma / \mathrm{d} M_{\gamma \gamma}$ for $\mathrm{pp} \rightarrow \gamma \gamma \mathrm{X}$ is shown in fig. 6 , for a typical cut on the angles of the $\gamma$ 's in their center of mass. It should be noted that the signal is reduced less rapidly as a function of the maximum $\left|\cos \theta_{\gamma}\right|$ allowed than is the background. We have chosen a reasonable value that retains a large fraction of the signal events. Given $\mathrm{d} \sigma / \mathrm{d} M_{\gamma \gamma}$, the actual background for a Higgs boson search is determined by the detector mass resolution. This issue has been studied most recently as part of the Snowmass 1986 and Madison 1987 workshops, see ref. [35]. Based upon these studies it appears to be entirely reasonable to expect a $1 \%$ to $2 \%$ 
resolution in $M_{\gamma \gamma}$, and, indeed, in any invariant mass constructed from 2 or 3 isolated purely electromagnetic tracks, provided the detector goals include reasonable optimization for this type of channel. Crudely, this is equivalent to having electromagnetic calorimeter resolution of $1 \%+10 \% / \sqrt{E}$, a form that has been used in several studies. We will adopt the procedure of considering signal and background integrated over a mass bin of width $0.02 m_{\mathrm{H}^{0}}$, centered at $m_{\mathrm{H}^{0}}$. As mentioned earlier, since the Higgs width is considerably narrower than this in the mass region of interest, virtually no reduction in the Higgs signal results from such a restriction.

Another background in this channel arises from the process $\mathrm{gg} \rightarrow \gamma \gamma$, which occurs through quark loops. Because the gluon structure functions are so large, this background might be comparable to that from $q \bar{q} \rightarrow \gamma \gamma$ [36]. We will include this

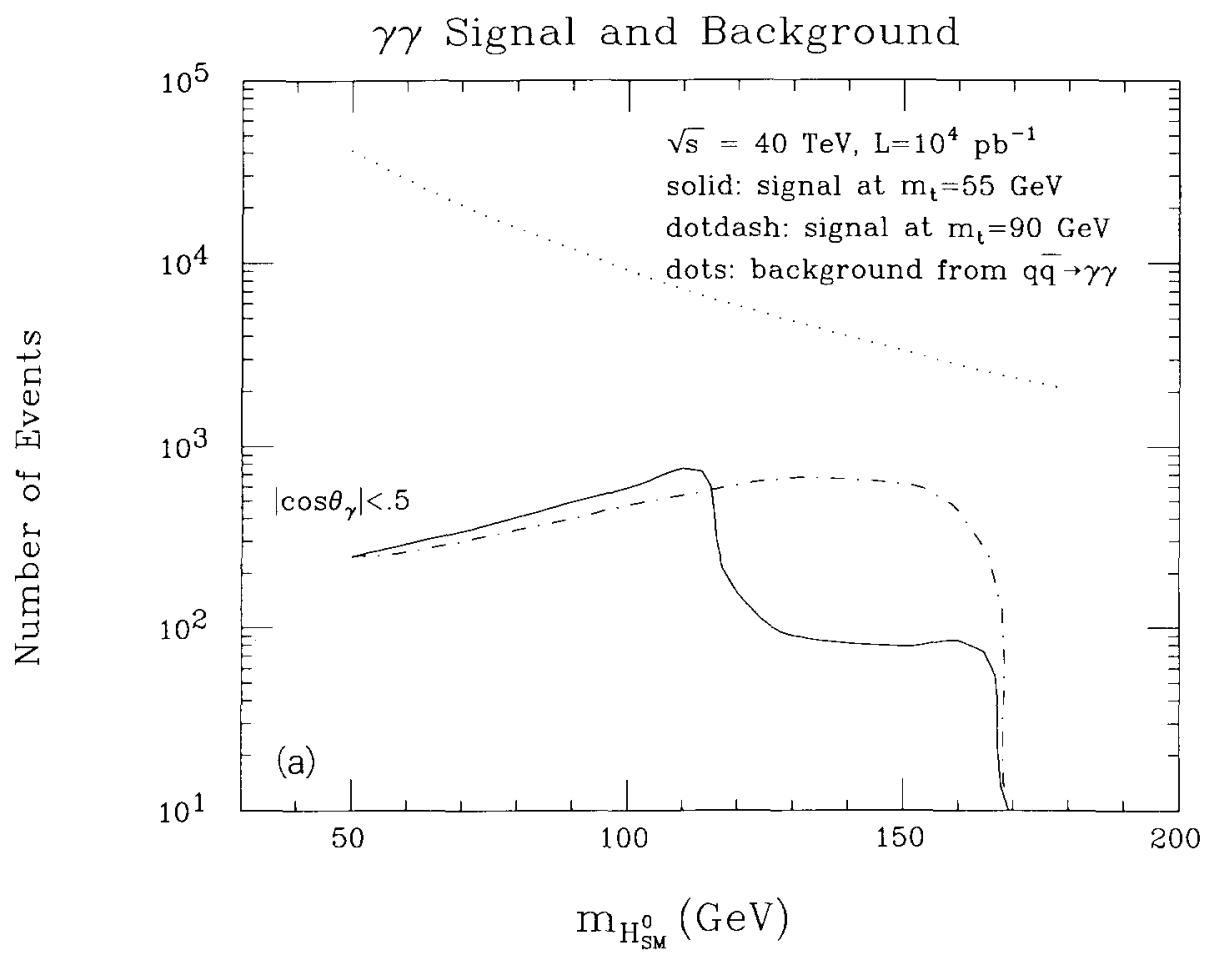

Fig. 7. Comparisons of signal and background rates for standard SSC luminosity as a function of $m_{\mathrm{H}_{S}^{0} \mathrm{M}}$ for $m_{\mathrm{t}}=55$ and $90 \mathrm{GeV}$, assuming a $2 \%$ mass bin in reconstructing the Higgs mass. Results for the Higgs signal are for the $\mathrm{SM} \mathrm{H} \mathrm{SM}_{\mathrm{S}}^{0}$ and would have to be altered in typical extended models of the Higgs sector. Branching ratio(s) to the purely electromagnetic final states of interest are included. Any additional cuts imposed are indicated on the graphs themselves. The channels appear in the order: $\gamma \gamma, Z_{\gamma}, Z^{*}$, and $\Theta \gamma$. In the $\gamma \gamma$ case the background is only that from $q \bar{q} \rightarrow \gamma \gamma$. In the $\Theta_{\gamma}$ case note that the plotted background curve is the background event rate divided by a factor of 16 . 


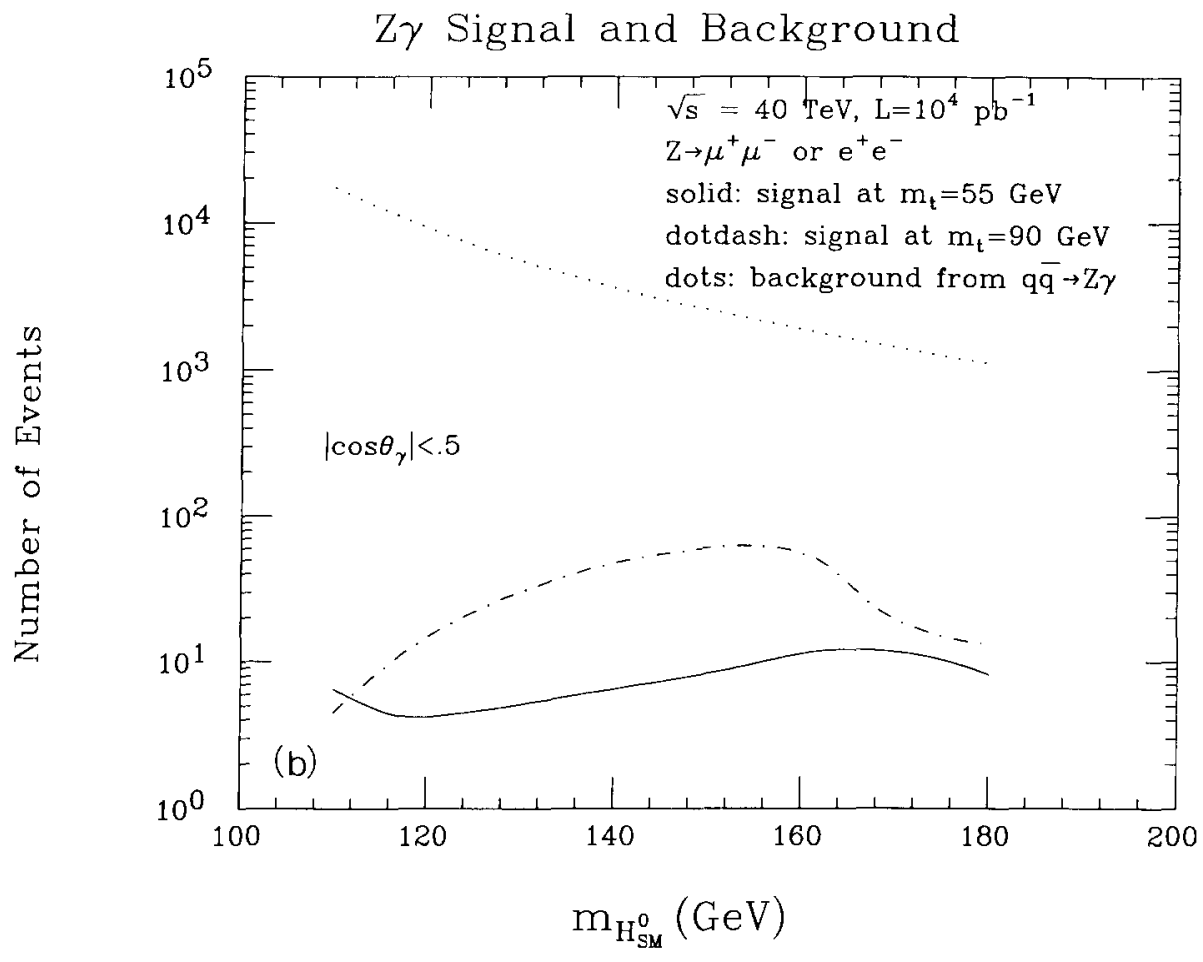

Fig. 7 (continued).

background, in assessing the utility of the $\gamma \gamma$ mode, by multiplying the $q \bar{q} \rightarrow \gamma \gamma$ background by a factor of 2 .

In order to assess the impact of the $\gamma \gamma$ background upon the $\gamma \gamma$ mode for Higgs detection, we plot in fig. 7 a the signal and $\mathrm{q} \overline{\mathrm{q}} \rightarrow \gamma \gamma$ background rates (assuming a $2 \%$ mass bin in the $\gamma \gamma$ channel) for several choices of $m_{\mathrm{t}}$. A cut on the $\gamma \gamma$ center of mass angle of the outgoing photons with respect to the beam direction of $\left|\cos \theta_{\gamma}\right|<$ 0.5 has been imposed in order to reduce the background relative to the signal. This type of cut is more effective than cutting only on the laboratory rapidity $y_{\gamma}$ of the outgoing photons. Of course, detector acceptance may be such that only a certain region of $y_{\gamma}$ for the photons is useful. Taking the restriction $\left|y_{\gamma}\right| \leqslant 4$ for both photons as an example, we find the following representative results with the $\cos \theta_{\gamma}$ cut already imposed; at $m_{\mathrm{H}^{0}}=50 \mathrm{GeV}$ the additional $y_{\gamma}$ cut reduces the background by another $25 \%$ but the signal by only $12 \%$; at $m_{\mathrm{H}^{0}}=140 \mathrm{GeV}$ the $y_{\gamma}$ cut reduces the background by $18 \%$ but the signal by only $7 \%$. Thus, such an additional restriction on the allowed range of $y_{\gamma}$ would actually improve the signal to background ratio, at the sacrifice of some event rate. However, our later analysis of 


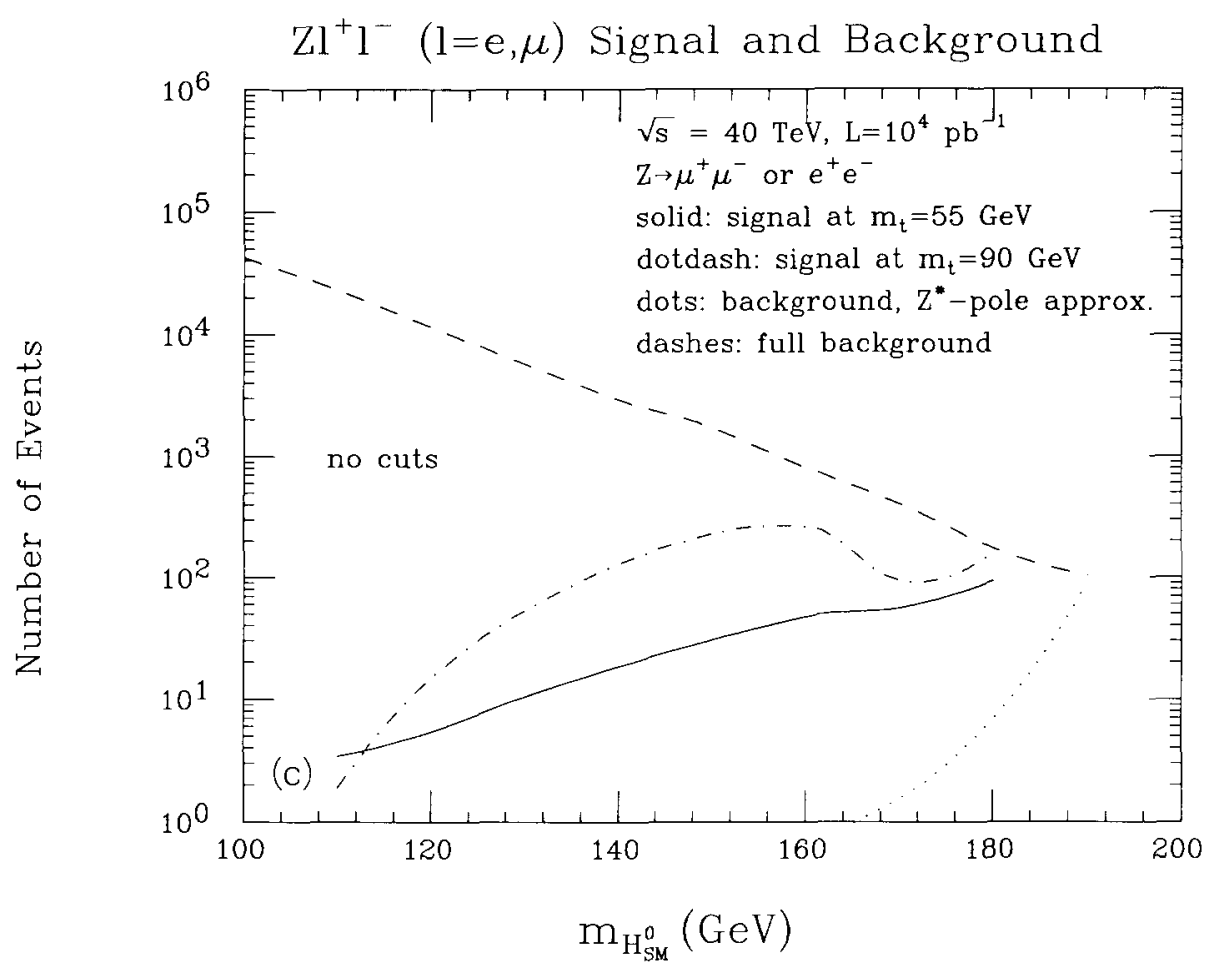

Fig. 7 (continued).

the statistical significance of the signal will be based on imposing only the $\cos \theta_{\gamma}$ cut; further optimization might be possible.

Another source of $\gamma \gamma$ events, which is not a background but rather provides a useful calibration source, is $\gamma \gamma$ pairs from the decay of tt onia states. In ref. [37] it is estimated that the cross section times $\gamma \gamma$ branching ratio for the decay of the $\eta_{1}$ para-quarkonium state is of order $10-1.5 \mathrm{pb}$ (using a branching ratio of $0.5 \%$ ) in the mass region of $100-180 \mathrm{GeV}$, i.e. one to two orders of magnitude larger than the expected Higgs boson signal; it should be easily detectable above backgrounds. By the time the SSC is running, there is a good chance that the toponium mass will be known, so that finding this mode will serve to calibrate and normalize the detector capabilities for $\gamma \gamma$ spectroscopy. If toponium is not found at Tevatron I or elsewhere, the $\gamma \gamma$ mode may be the best way to discover it at the SSC. Other toponium states (in addition to the $\eta_{t}$-state) have $\gamma \gamma$ modes, so the full rates to be expected can only be determined by detailed calculations; the ratio of $\sigma \times \operatorname{BR}(\gamma \gamma)$ for a Higgs boson to that for a t-onia state can probably be calculated rather accurately. (With a 2\% mass resolution the various t-onia levels cannot, however, be 


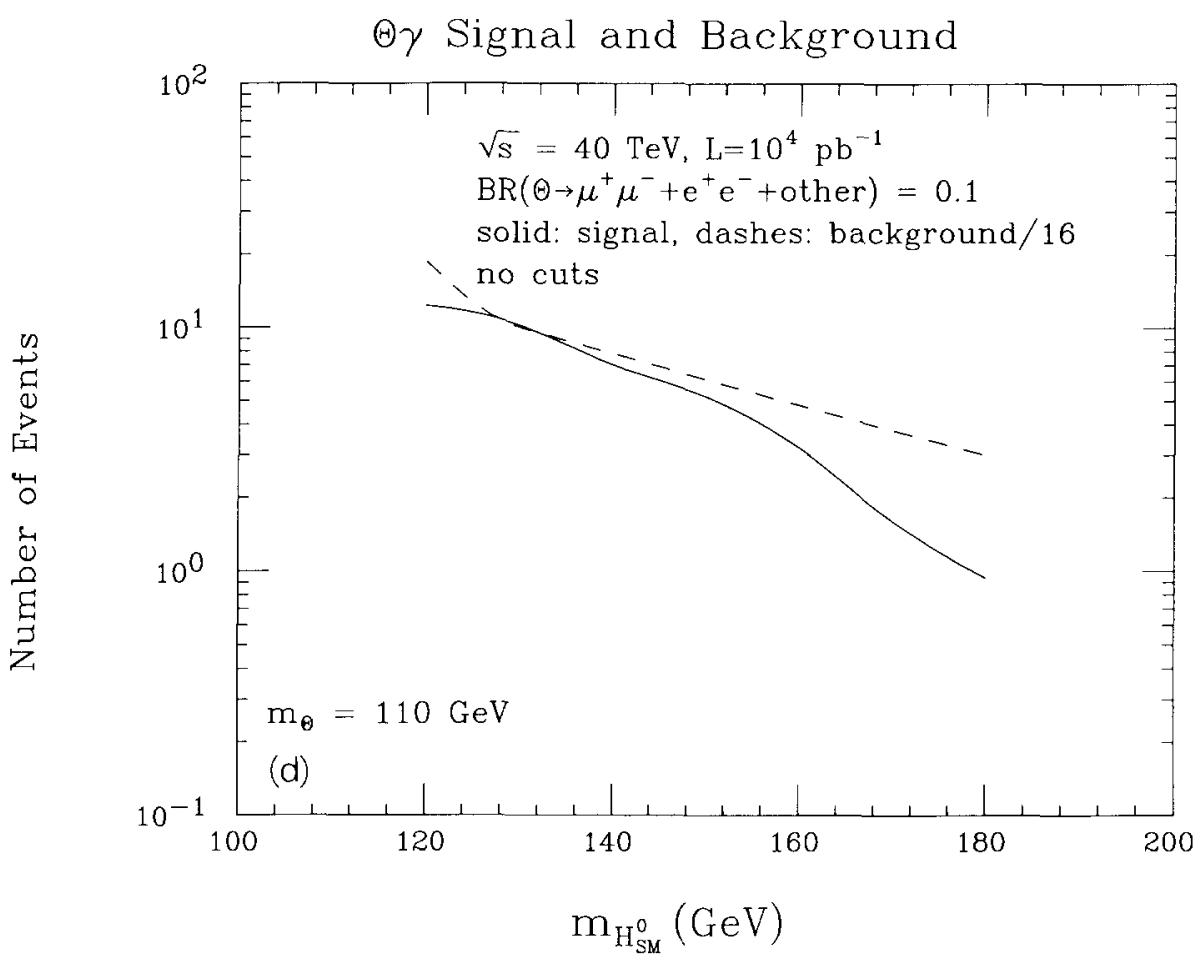

Fig. 7 (continued).

separated.) Angular distributions for the $\gamma \gamma$ from the two different types of decays can be compared.

The above backgrounds are intrinsic ones which can at best be reduced by mass resolution and angular cuts. In addition to these, there is a potentially very serious background from a jet faking a $\gamma$ in the detector. It is extremely important that the detector be designed to optimize $\gamma$-jet separation, since isolated hard $\gamma$ 's not only seem to provide the best way to search for intermediate mass Higgs bosons, but also could provide a signature for supersymmetry or compositeness. Detailed Monte Carlo's are required to determine precisely what degree of discrimination between photons and jets is required. We estimate (see also ref. [35]) that the ratio of $\sigma(j j)$ to $\sigma(\gamma \gamma)$ (the latter coming from a Higgs decay) is about $0.5 \times 10^{8}$ for the mass resolutions being considered. Thus a discrimination of about $4-5 \times 10^{-5}$ should suffice. Some studies of this issue were done for the Snowmass 1986 workshop [38], with generally favorable conclusions. A critical analysis of this work is necessary [39]. However, we believe that ultimately it will be demonstrated that this discrimination level is possible, on the basis of two indications. First, the UA2 group 
has published [40] a search for $\gamma+$ jet events at the CERN collider, obtaining a $\gamma /$ jet discrimination of $10^{-4}$ (on a statistical basis) in a detector that was not initially optimized for such studies, and at somewhat lower $p_{\mathrm{T}}$ values than are of interest for intermediate mass Higgs. Second, some of the Monte Carlo studies at Snowmass 1986 suggested that a few $\times 10^{-5}$ separation of $\gamma$ from jet was possible [38].

2.4.2. $H^{0} \rightarrow Z_{\gamma}$. The sources of background for the $Z_{\gamma}$ channel are very similar to those that are present in the $\gamma \gamma$ channel. Backgrounds arise from $\mathrm{q} \overline{\mathrm{q}} \rightarrow \mathrm{Z} \gamma, \mathrm{gg} \rightarrow$

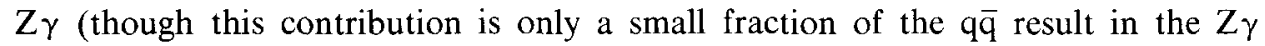
channel case), and from $Z+$ jet sources. Again, the t-onia decays to $Z \gamma$ may provide a useful calibration. As for the $\gamma \gamma$ case, we plot in fig. 7 the signal and background rates in this channel, for two $\mathrm{m}_{\mathrm{t}}$ choices, assuming a $2 \%$ mass bin in the $\ell^{+} \ell^{-} \gamma$ final state. A cut on the angle of the outgoing $\gamma$ in the $Z \gamma$ center of mass of $\left|\cos \theta_{\gamma}\right|<0.5$ has been imposed. Obviously the signal to background ratio is much smaller than for the $\gamma \gamma$ channel. Nevertheless, if the $\mathrm{H}^{0}$ is detected in another mode, it should be possible to observe a low statistics signal at the given mass in $Z \gamma$, and therefore to use this mode to help determine couplings and loop effects.

2.4.3. $H^{0} \rightarrow \Theta \gamma$. One technique for estimating this background is to employ the formalism of ref. [31], which is quite successful in describing $\Psi \mathrm{g}$ and $\mathrm{rg}$ data, when the invariant mass of the final state is somewhat larger than the onium mass itself. We have computed [41] the invariant amplitude squared arising from the (six) contributing diagrams for $\mathrm{gg} \rightarrow \Theta \gamma$, and obtained by Monte Carlo integration the resulting cross section for a $2 \%$ mass bin about a given invariant mass for the $\Theta \gamma$ system. No rapidity cuts have been incorporated because of the low signal event rate. The resulting event rates for signal and background are given in fig. 7 , for $m_{\mathrm{t}}=55 \mathrm{GeV}$. We have taken a combined branching ratio of $10 \%$ for those $\Theta$ channels that can be measured with $\sim 2 \%$ mass resolution. This calculation suggests that the background is substantially larger than the signal over the intermediate mass range.

However, as a check on the calculation we have also estimated the background rate in this channel from the reported UA1 measurement [42] of the rate for $\mathrm{p} \overline{\mathrm{p}} \rightarrow \Upsilon, \Upsilon^{\prime}, \Upsilon^{\prime \prime}, \ldots+\mathrm{X}$, using the decays of the $\Upsilon$ states to $\mu^{+} \mu^{-}$. They obtain $\sigma=405 \mathrm{pb}$, with about a $30 \%$ error. A cut of $p_{\mathrm{T}}^{\mu}>3 \mathrm{GeV}$ was imposed, but no cut on $p_{\mathrm{T}}^{\gamma}$. We can then estimate the $\Theta \gamma$ background by: (i) assuming that the presence of the extra hard isolated $\gamma$ should cost a factor of $\leqslant \frac{1}{100}$; (ii) assuming that the scaling of cross sections goes as $M_{\text {onia }}^{-2}$ implying a factor of $\leqslant \frac{1}{100}$ in going from $r$ to $\Theta$; and (iii) requiring some $p_{\mathrm{T}}$ for the $\Theta$ yielding a factor of $\sim \frac{1}{3}$. The resulting cross section of $0.03 \mathrm{pb}$ must be further reduced for the requirement that only a $2 \%$ mass bin will be accepted. If this results in a factor of $\sim \frac{1}{30}$ we are left with a number of events $(\sim 10)$ which is substantially smaller than that obtained by the analytic calculation. At the moment, we are unsure of which method of approximating the background should be considered most reliable. Optimistically, the $\Theta \gamma$ mode 
could provide a useful confirmation of intermediate mass Higgs discovery in another mode.

2.4.4. $H^{0} \rightarrow Z Z^{*}$. As for the previous modes, all the relevant backgrounds must be considered. It is, of course, important to recall that both the $Z$ and $Z^{*}$ will be observed only in their charged leptonic decay channels. One might hope that a rough idea of the primary background, continuum $\mathrm{q} \overline{\mathrm{q}} \rightarrow \mathrm{Z}^{+} \ell^{-}$, could be obtained by using the approximate form given in ref. [18], found by keeping only the virtual $\mathrm{Z}^{*}$ poles in the $\ell^{+} \ell^{-}$channel. This approximation is shown in fig. 7c. However, a full calculation of the background should include all eight contributing diagrams, in particular those with $\gamma^{*}$ virtual poles. The complete matrix element was computed for the study of ref. [18] and we have evaluated the background for the situation of interest here. The result is shown in fig. $7 \mathrm{c}$, in the absence of any cuts. Obviously the full uncut background is rather large, and the $Z^{*}$-pole approximation is not at all good once one moves away from the $\mathrm{Z}$ pole. (When plotting signal and background rates we have, as in the other cases, assumed a $2 \%$ mass bin and standard SSC yearly luminosity.) However, it is intuitively obvious that much of this full background arises from the $\gamma^{*}$ poles when the mass of the $\ell^{+} \ell^{-}$system, $M_{\ell^{+} \ell^{-}}$, is small. In contrast the signal will obviously peak for large $M_{\ell^{+} \ell^{-}}$since it contains only a $Z^{*}$ pole. This is dramatically illustrated in fig. 8 , where we present the $M_{\ell^{+} \ell}$. distributions for the full background and the Higgs boson signal in the case of $m_{\mathrm{H}_{\mathrm{S}}^{0}}=M_{\mathrm{Z}^{+} \ell^{-}}=140 \mathrm{GeV}$. Obviously, requiring that $M_{\ell^{+} \ell^{-}}$be larger than some value (e.g. $20 \mathrm{GeV}$ for the present case) will dramatically reduce the background.

In table 1 we present a compilation of typical $M_{\ell^{+} \ell^{-}}$cuts for a number of $m_{\mathrm{H}_{\mathrm{SM}}^{0}}$ values, along with the resulting background and signal event rates (at both $m_{\mathrm{t}}=55$ $\mathrm{GeV}$ and $m_{\mathrm{t}}=90 \mathrm{GeV}$ for the latter). In all cases, the cut dramatically reduces the background while being at least $85 \%$ efficient in retaining signal events.

Note that after the $M_{\ell^{+} \ell^{-}}$cut, the viability of the $\mathrm{ZZ}^{*}$ channel is essentially determined by the raw signal event rate given in fig. $7 \mathrm{c}$, multiplied by an efficiency factor of order $90 \%$.

From the table we see that the range in $m_{\mathrm{H}_{\mathrm{SM}}^{0}}$ for which this channel is viable depends substantially on the $t$ quark mass. Only if the Higgs does not decay to $\bar{t} \bar{t}$ can we obtain a significant signal for $m_{\mathrm{H}_{\mathrm{SM}}^{0}}$ as low as $\sim 120 \mathrm{GeV}$. At $m_{\mathrm{t}}=55 \mathrm{GeV}$ the range of detection for a standard SSC year extends only down to $\sim 140 \mathrm{GeV}$. It is important to note that in either case this channel does allow detection in the region $2 m_{\mathrm{W}} \leq m_{\mathrm{H}_{\mathrm{S}}^{0}} \leq 2 m_{\mathrm{Z}}$, where it is unlikely that the WW channel can be employed, despite its rapidly becoming the dominant decay mode. Finally, we remark that since the background in this case is generally significantly smaller (after the $M_{\ell^{+} \ell^{-}}$cut) than the signal, and since the final state should be very clean in a detector sense, this mode would benefit greatly from an interaction region with as large a luminosity as possible. At $L=10^{5} \mathrm{pb}^{-1}$ this decay mode could be used to search for a SM Higgs all the way down to $120 \mathrm{GeV}$ at $m_{\mathrm{t}}=55 \mathrm{GeV}$ and possibly as low as $100 \mathrm{GeV}$ at $m_{\mathrm{t}}=90 \mathrm{GeV}$. 


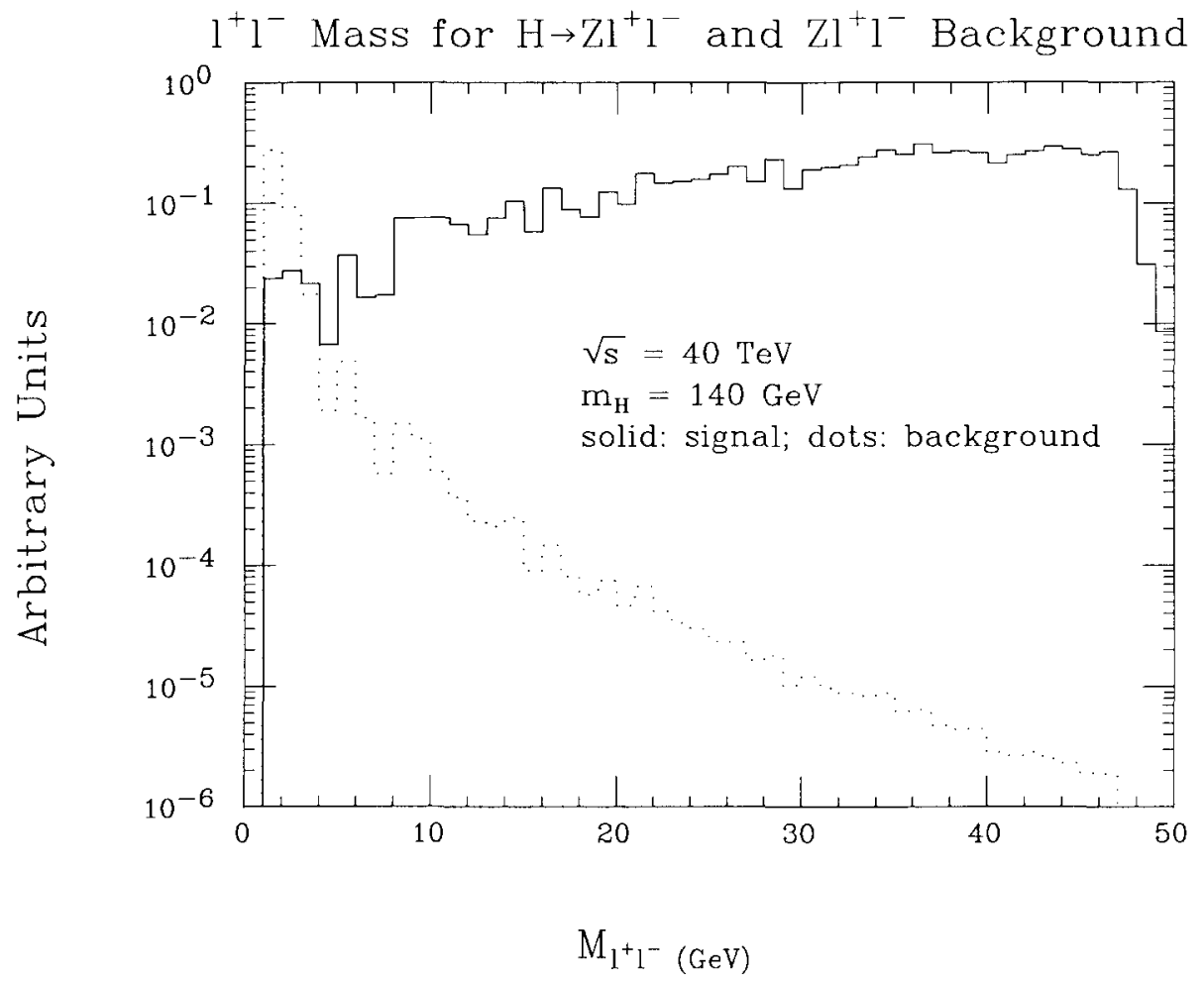

Fig. 8. We plot, for $M_{\mathrm{Z}^{+} \ell^{-}}=140 \mathrm{GeV}$, in arbitrary units, the $M_{\ell^{+} \ell^{-}}$distributions for the full $\mathrm{Z}^{+} \ell^{-}$ background and the $\mathrm{H}_{\mathrm{SM}}^{0} \rightarrow \mathrm{Z} \ell^{+} \ell^{-}$signal.

TABLE 1

$M_{\ell^{+} \ell^{-}}$cuts: Associated signal and background event rates

\begin{tabular}{ccccc}
\hline$m_{\mathrm{H}_{\mathrm{SM}}^{0}}(\mathrm{GeV})$ & $M_{\ell^{+} \ell^{-} \text {cut }}$ & $m_{\mathrm{t}}=55 \mathrm{GeV}$ & $m_{\mathrm{t}}=90 \mathrm{GeV}$ & Background \\
\hline 120 & $10 \mathrm{GeV}$ & 3 & 13 & 2 \\
140 & $20 \mathrm{GeV}$ & 16 & 110 & 3 \\
160 & $25 \mathrm{GeV}$ & 44 & 248 & 2 \\
170 & $30 \mathrm{GeV}$ & 54 & 87 & 2 \\
180 & $40 \mathrm{GeV}$ & 84 & 143 & 8 \\
\hline
\end{tabular}

2.4.5. Results for the $S M H_{S M}^{o}$. We now employ the event rates obtained following the examples and discussion of the previous section to assess the statistical significance of the Higgs boson cross section in each of the various channels, $\mathrm{C}$. We consider each point in the $m_{\mathrm{t}}-m_{\mathrm{H}_{\mathrm{SM}}^{0}}$ plane, comparing $\sigma\left(\mathrm{pp} \rightarrow \mathrm{H}_{\mathrm{SM}}^{0}+\mathrm{X}\right) \mathrm{BR}\left(\mathrm{H}_{\mathrm{SM}}^{0}\right.$ $\rightarrow$ C) with a bin of width $\Delta M_{\mathrm{C}}=0.02 M_{\mathrm{C}}$ at $M_{\mathrm{C}}=m_{\mathrm{H}_{\mathrm{SM}}^{0}}$ in the cross section $\mathrm{d} \sigma(\mathrm{pp} \rightarrow \mathrm{C}) / \mathrm{d} M_{\mathrm{C}}$. We then divide the $m_{\mathrm{t}}-m_{\mathrm{H}_{\mathrm{SM}}^{\mathrm{o}}}$ plane into two regions separated 
by a boundary specified by those points where a four standard deviation effect in signal relative to background is achieved. That is, if the number of events in the signal is, for luminosity $L$ and running time $T$,

$$
S=\sigma\left(\mathrm{pp} \rightarrow \mathrm{H}_{\mathrm{SM}}^{0}+\mathrm{X}\right) \times \mathrm{BR}\left(\mathrm{H}_{\mathrm{SM}}^{0} \rightarrow \mathrm{C}\right) \times L \times T
$$

and the background is

$$
B=\frac{\mathrm{d} \sigma(\mathrm{pp} \rightarrow \mathrm{C}+\mathrm{X})}{\mathrm{d} M_{\mathrm{C}}} \Delta M_{\mathrm{C}} \times L \times T,
$$

then the boundary is the line where

$$
\frac{S}{4 \sqrt{B}}=1
$$

Above the boundary a Higgs should be detectable based on that channel alone, while below the boundary it probably is not. Of course, a combination of all the channels could also be considered.

We focus first on the $\gamma \gamma$ channel. The boundary curve for this case is shown in fig. 9. We have included the gg $\rightarrow \gamma \gamma$ contribution to the background by doubling the $q \bar{q} \rightarrow \gamma \gamma$ background contribution. We have enforced a requirement of $\left|\cos \theta_{\gamma}\right|$ $<0.5$ on the outgoing $\gamma$ 's in the $\gamma \gamma$ center of mass frame, where $\theta_{\gamma}$ is measured with respect to the beam direction. Restricted detector coverage in overall laboratory rapidity results in some additional decrease of both signal and background. However, as discussed earlier, the signal decreases less rapidly than the background as the maximum allowed $\left|y_{\gamma}\right|$ decreases, and the statistical significance improves slightly relative to the results we quote, so long as the $y_{\gamma}$ restriction is not too severe.

The same type of analysis can be repeated for the $Z \gamma$ channel, where we require that the $\mathrm{Z}$ be observed in its $\mathrm{e}^{+} \mathrm{e}^{-}$or $\mu^{+} \mu^{-}$decay mode. Using fig. $7 \mathrm{~b}$, and analogous results at other $m_{\mathrm{t}}$ values, we are unable to find a region where the statistical significance is $>4 \sigma$, even for the $5 \times 10^{4} \mathrm{pb}^{-1}$ integrated luminosity. For instance, at $m_{\mathrm{t}}=90 \mathrm{GeV}$ and using the above enhanced luminosity, the best statistical significance is achieved in the vicinity of $m_{\mathrm{H}_{S M}^{0}} \sim 160 \mathrm{GeV}$ where we obtain an $\sim 3.5 \sigma$ effect. Thus we must view the $Z \gamma$ decay channel as, at best, a possible means of confirming Higgs discovery in some other rare decay mode.

A different type of analysis is appropriate to the $\mathrm{ZZ}^{*}$ channel. As we have seen, a cut in $M_{\ell^{+} \ell^{-}}$can be chosen at each $m_{\mathrm{H}_{\mathrm{SM}}^{0}}$ value such that the background is essentially negligible compared to the signal, while retaining of order $90 \%$ of the signal. Thus, we shall adopt the procedure of establishing our boundaries by requiring that there be 40 events in the uncut signal, where, of course, we require that both the $\mathrm{Z}$ and $\mathrm{Z}^{*}$ decay to $\mathrm{e}^{+} \mathrm{e}^{-}$or $\mu^{+} \mu^{-}$. This number is chosen conserva- 


\section{$\gamma \gamma$ Mode $4 \sigma$ Contours}

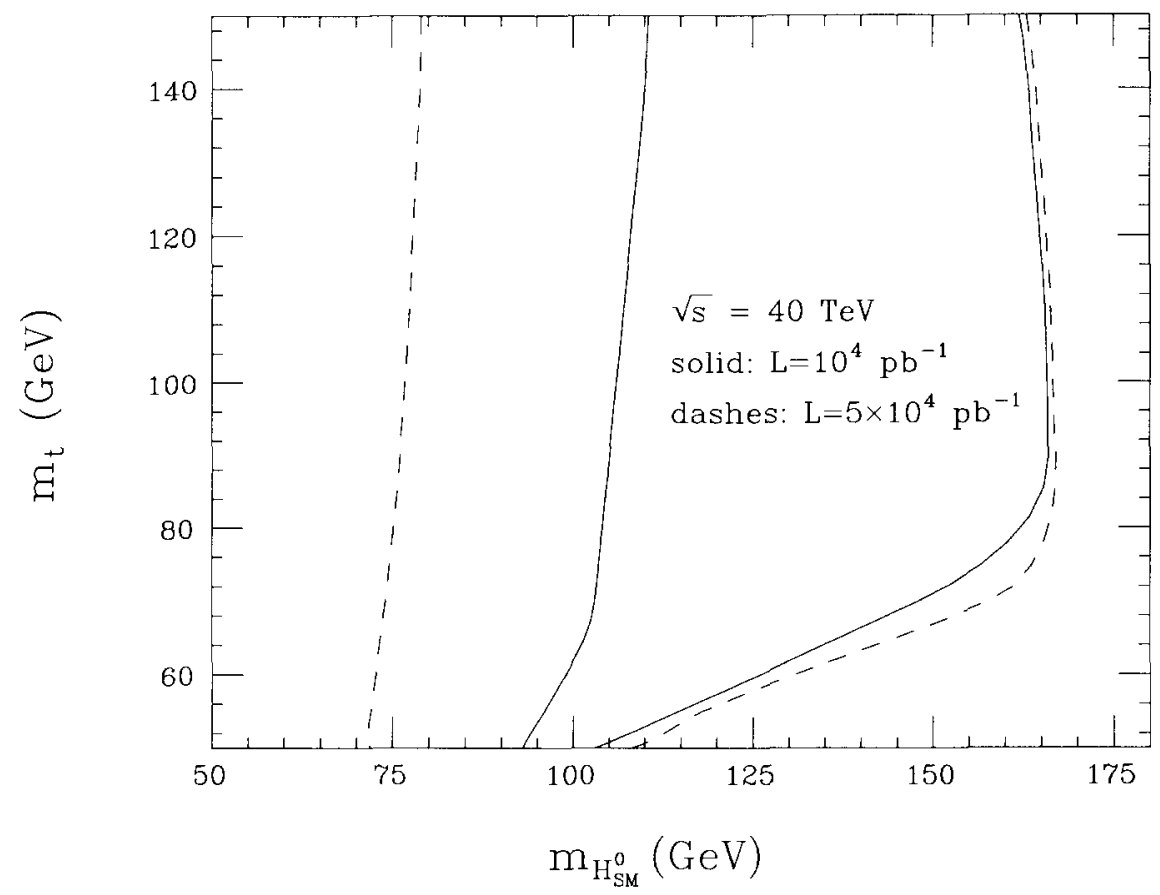

Fig. 9. We give the boundaries in $m_{\mathrm{t}}-m_{\mathrm{H}_{\mathrm{SM}}^{0}}$ space which separate the region in which the $\gamma \gamma$ mode yields a $>4 \sigma$ effect from that where it does not. The two different boundaries correspond to integrated luminosities of $L=10^{4} \mathrm{pb}^{-1}$ and $5 \times 10^{4} \mathrm{pb}^{-1}$. A $2 \%$ mass bin is assumed for the $\gamma \gamma$ final state, and a cut on the angle with respect to the beam of the outgoing photons in their center of mass of $\left|\cos \theta_{\gamma}\right|<0.5$ is made. The $\mathrm{q} \overrightarrow{\mathrm{q}} \rightarrow \gamma \gamma$ background is doubled to account for $\mathrm{gg} \rightarrow \gamma \gamma$.

tively so as to allow for the reduction in the number of events deriving from the $M_{\ell^{+} \ell^{-}}$cuts and realistic detector acceptance for the final state leptons. The resulting boundaries at $L=10^{4} \mathrm{pb}^{-1}$ and $L=5 \times 10^{4} \mathrm{pb}^{-1}$ are displayed in fig. 10.

By examining figs. 9 and 10 we may now assess the sensitivity of the SSC to a Higgs of a given mass as a function of $m_{\mathrm{t}}$. Let us assume standard SSC yearly luminosity of $L=10^{4} \mathrm{pb}^{-1}$, and consider various $m_{\mathrm{t}}$ regions. Suppose, for example that $m_{\mathrm{t}} \geq 80 \mathrm{GeV}$. Then fig. 9 indicates that the $\gamma \gamma$ mode can cover the region $-105 \mathrm{GeV} \leq m_{\mathrm{H}_{\mathrm{SM}}^{0}} \leq 2 m_{\mathrm{W}}$. To confirm a signal in this region and to cover the rest of the intermediate mass region we must employ at least two other modes. For instance, for $m_{\mathrm{t}} \geq 80 \mathrm{GeV}$, a SM Higgs with $\sim 125 \mathrm{GeV} \leqslant m_{\mathrm{H}_{\mathrm{SM}}^{0}} \leqslant 2 m_{Z}$ can be detected using the $Z Z Z^{*}$ decay mode, with both the $\mathrm{Z}$ and $\mathrm{Z}^{*}$ decaying to $\mathrm{e}^{+} \mathrm{e}^{-}$or $\mu^{+} \mu^{-}$. This is indicated by the boundary curves for this mode displayed in fig. 10 . Thus the ZZ* mode covers the region above $2 m_{\mathrm{W}}$ and would provide confirmation 


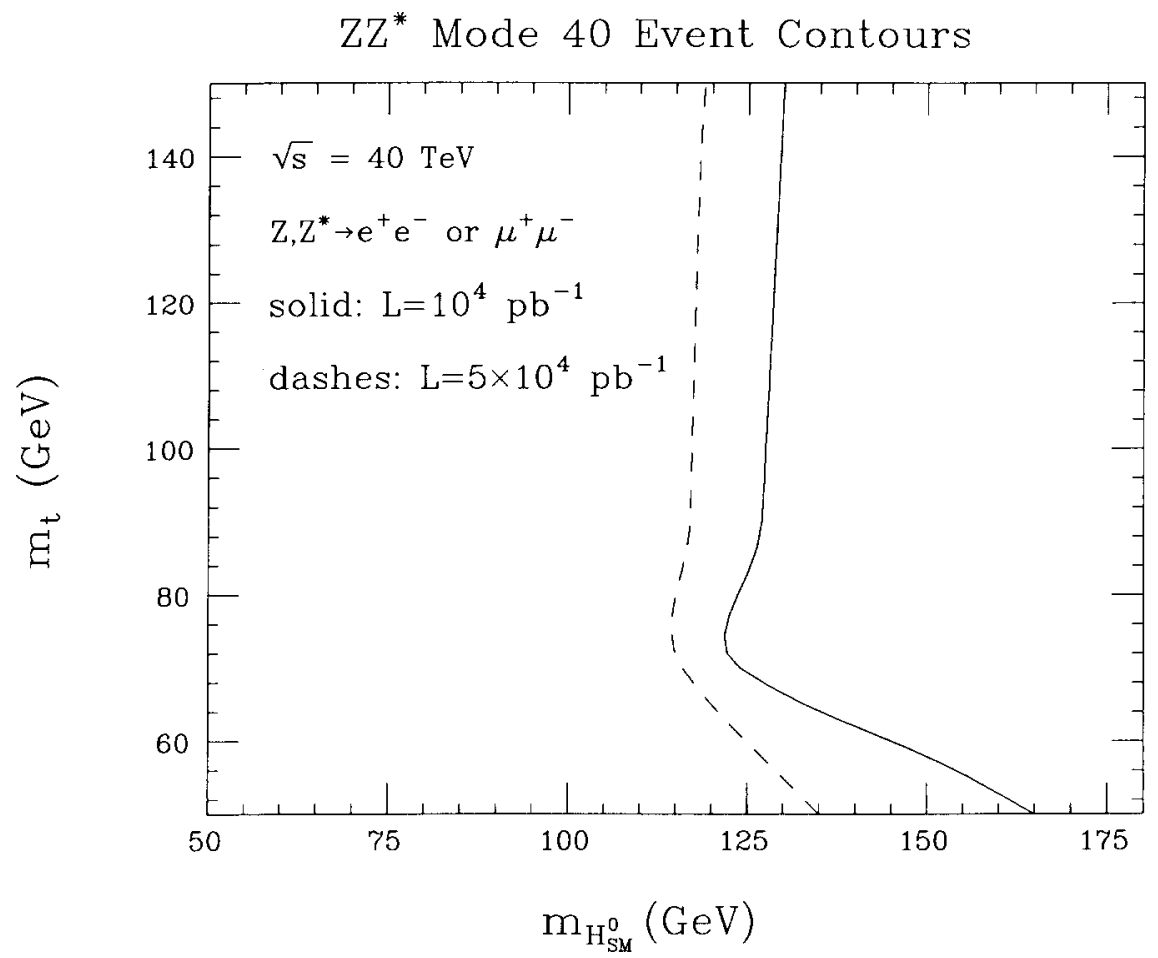

Fig. 10. We present the boundary curves for the $\mathrm{ZZ}^{*}$ mode which separate the region where the number of Higgs decay events is $>40$ from that where it is $<40$. Both the $Z$ and $Z^{*}$ are required to decay to $\mathrm{e}^{+} \mathrm{e}^{-}$or $\mu^{+} \mu^{-}$. No cuts are imposed. We give boundaries for $L=10^{4} \mathrm{pb}^{-1}$ and $L=5 \times 10^{4} \mathrm{pb}^{-1}$.

of a $\gamma \gamma$ signal in the region between $125 \mathrm{GeV}$ and $2 m_{\mathrm{W}}$. The region $0.5 m_{\mathrm{Z}} \leqslant m_{\mathrm{H}_{\mathrm{SM}}^{0}}$ $\leqslant 105 \mathrm{GeV}$ is also not covered by $\gamma \gamma$. But in this region, and, indeed, for all $m_{\mathrm{H}_{\mathrm{SM}}^{0}}<2 m_{\mathrm{t}}$, the dominant decay of the $\mathrm{H}_{\mathrm{SM}}^{0}$ is to $\mathrm{b} \bar{b}$. The detection technique originally suggested in ref. [17] and elaborated upon in refs. [18], in which one looks for associated production of the $\mathrm{H}_{\mathrm{SM}}^{0}$ with a charged $\mathrm{W}$, followed by decay of the $\mathrm{H}_{\mathrm{SM}}^{0}$ to its dominant $\mathbf{f}$ channel, could become viable for $\mathbf{f}=b$, as explored in ref. [20], even though it was not viable for $\mathrm{f}=\mathrm{t}$ due to the degradation in mass resolution from the multistage top decays [19]. Thus this associated production mode can probably be used to cover the lower $m_{\mathrm{H}_{\mathrm{SM}}^{0}}$ region and to confirm a $\gamma \gamma$ signal for all $m_{\mathbf{H}_{\mathrm{SM}}^{0}}<2 m_{\mathrm{t}}$. Further Monte Carlo study is warranted, especially in the region of $m_{\mathrm{H}_{\mathrm{SM}}^{0}} \sim m_{\mathrm{Z}}$ where $\mathrm{WZ}$ backgrounds could become a problem, and at low $m_{\mathrm{H}_{\mathrm{SM}}^{0}}$ where mixed QCD/electroweak $\mathrm{W} b \overline{\mathrm{b}}$ backgrounds become larger. However, we tentatively conclude that for $m_{\mathrm{t}} \geqslant 80 \mathrm{GeV}$ the entire intermediate mass region can be covered at the SSC using a combination of the associated production, $\mathrm{ZZ}^{*}$, and $\gamma \gamma$ detection modes. Further, in the portion of $m_{\mathrm{H}_{\mathrm{SM}}^{0}}<2 m_{\mathrm{t}}$ for which the $\gamma \gamma$ mode is viable, confirmation from associated production is likely, while at higher 
$m_{\mathbf{H}_{S M}^{o}}$ values there is a substantial overlap between the viable regions for the $\gamma \gamma$ and $\mathrm{ZZ}^{*}$ modes.

As $m_{\mathrm{t}}$ decreases below $\sim 80 \mathrm{GeV}$, the region covered by the $\gamma \gamma$ mode becomes smaller, and almost disappears by $m_{\mathrm{t}} \sim 50 \mathrm{GeV}$, where the $m_{\mathbf{H}_{\mathrm{SM}}^{\mathrm{y}}} \sim 2 m_{\mathrm{t}}$ approximate upper limit on the $\gamma \gamma$ mode almost joins the lower limit deriving from decreasing event rate versus background. A substantial segment of the $m_{\mathrm{H}_{\mathrm{SM}}^{0}}$ mass region below the smaller of $2 m_{\mathrm{t}}$ and the above lower limit may again be covered by $\mathrm{WH}_{\mathrm{SM}}^{0}$ associated production with $\mathrm{H}_{\mathrm{SM}}^{0} \rightarrow \mathrm{b} \bar{b}$, though, as mentioned previously, at these lower masses Monte Carlo analysis is needed for confirmation. From fig. 10 we see that the smallest value of $m_{\mathrm{H}_{\mathrm{S}}^{0}}$ that may be detected using the $\mathrm{ZZ}{ }^{*}$ mode also increases as $m_{\mathrm{t}}$ drops below $\sim 70 \mathrm{GeV}$; this minimum detectable $m_{\mathrm{H}_{\mathrm{S}}^{0}}$ eventually reaches $-165 \mathrm{GeV}$ at $m_{\mathrm{t}}=50 \mathrm{GeV}$. Thus we are left with a wedge in $m_{\mathrm{t}}-m_{\mathrm{H}_{\mathrm{SM}}^{0}}$ parameter space (considering only $m_{\mathrm{t}}>50 \mathrm{GeV}$ ), extending from $m_{\mathrm{H}_{\mathrm{SM}}^{0}}$ $\sim 110 \mathrm{GeV}$ to $\sim 165 \mathrm{GeV}$ at $m_{\mathrm{t}}=50 \mathrm{GeV}$ with apex at $m_{\mathrm{t}} \sim 65 \mathrm{GeV}, m_{\mathrm{H}_{\mathrm{SM}}^{0}} \sim 135$ $\mathrm{GeV}$, in which neither the $\gamma \gamma$ nor the $\mathrm{ZZ}^{*}$ mode can provide a fully satisfactory signal for the Higgs boson.

Of course, figs. 9 and 10 make it clear that a higher integrated luminosity will greatly reduce the size of this wedge. In addition, fig. 9 makes it evident that the $\gamma \gamma$ mode will be useful down to much lower values of $m_{\mathrm{H}_{\mathrm{SM}}^{0}}$ at higher $L$, e.g. down to $m_{\mathrm{H}_{\mathrm{S}}^{0}}-75 \mathrm{GeV}$ at $L=5 \times 10^{4} \mathrm{pb}^{-1}$. Further increases of the lower bound on $m_{\mathrm{t}}$ will also diminish the size of this problematical wedge.

However, should $m_{\mathrm{t}}$ and $m_{\mathrm{H}_{\mathrm{S}}^{0}}$ happen to fall within the above wedge, where the $\gamma \gamma, \mathrm{ZZ}^{*}$ and $\mathrm{WH}_{\mathrm{SM}}^{0}$ techniques are not adequate, it will probably be necessary to turn to the slightly less straightforward $\tau^{+} \tau^{-}$decay channel of the $\mathrm{H}_{\mathrm{SM}}^{0}$. The branching ratio to this channel does not fall as precipitously above the $\bar{t} \bar{t}$ threshold as do most of the other rare modes, and it begins at a higher level, as seen in fig. 1 . However, direct inclusive production of the $\mathrm{H}_{\mathrm{SM}}^{0}$ followed by $\mathrm{H}_{\mathrm{SM}}^{0} \rightarrow \tau^{+} \tau^{-}$yields poor $\tau^{+} \tau^{-}$mass resolution in the dominant single charged particle decays of the $\tau$ 's due to lost neutrinos and absence of constraints, so that inclusive $Z$ (and $Z^{*}$ ) production with decay to the $\tau^{+} \tau^{-}$channel provides a ferocious background [43]. Multiprong $\tau$ decays, despite their smaller branching ratio will yield better $\tau^{+} \tau$ mass resolution, and are being studied. Another possibility is to consider production modes for which the $\mathrm{H}_{\mathrm{SM}}^{0}$ has significant transverse momentum. For instance, if the $\mathrm{H}_{\mathrm{SM}}^{0}$ is produced in association with either a high- $p_{\mathrm{T}} \mathrm{Z}$ or gluon, the light mass of the $\tau$ 's in combination with the two transverse plane constraint equations, allows reconstruction of the $\tau^{+} \tau^{-}$mass, even in the single prong $\tau$ decay modes. This was originally pointed out in ref. [18]. A study at the Madison 1987 workshop, see ref. [43], suggests that a half width of about $30 \mathrm{GeV}$ could be achieved in the $\tau^{+} \tau^{-}$ reconstructed mass in the $m_{\mathrm{H}_{\mathrm{SM}}^{0}}$ mass region of interest. This would allow some possibility for $\mathrm{H}_{\mathrm{SM}}^{0}$ discovery, using the $\mathrm{Z}$ trigger, for a reasonable range above $2 \mathrm{~m}_{\mathrm{t}}$ [43]. Raw rates for the g trigger have been computed in ref. [44]. However, resolution is worse than in the $\mathrm{Z}$ trigger case due to miss-measurement of the $\mathrm{g}$ jet 
energy. Preliminary Monte Carlo results [43] suggest that the utility of this mode will not be much greater than that of the $Z$ trigger mode, despite the higher event rate. Further study, including real detector efficiencies for $\tau$ identification, is required.

Alternatively, for this light $m_{\mathrm{t}}$ situation, the $\Theta \gamma$ mode could be considered. While there is uncertainty in the exact level of the background in this case, it could turn out that signal and background rates are about equal and of the order of 10 events per SSC year. This would be marginal, but the mode could become very useful at a high luminosity integration region with 10 times the standard SSC yearly luminosity.

Overall, we conclude that, while considerable additional simulation work is required to be confident, it will probably be possible to cover the entire $m_{\mathrm{t}}-m_{\mathrm{H}_{\mathrm{SM}}^{0}}$ intermediate mass range plot $\left(m_{\mathrm{t}} \geq 50 \mathrm{GeV}\right)$, in the case of a SM Higgs boson, by combining a variety of modes, including $\gamma \gamma, \mathrm{b} \overline{\mathrm{b}}$ with associated $\mathrm{W}$ production, $\mathrm{ZZ}^{*}$, and $\tau^{+} \tau^{-}$with associated $Z$ or $g$ production. In addition $Z \gamma$ and $\Theta \gamma$ can be detected over much of the region where they are allowed, and should provide a supporting indication of the presence of the Higgs resonance. Together, these modes can be used for a determination of most of the important Higgs boson couplings, thus providing powerful clues to the origin of the Higgs mechanism.

For non-standard neutral Higgs, e.g. the $h$ of supersymmetry, branching ratios can vary from those of fig. 1 . In many cases, though not necessarily all, the rare decay mode BR's are larger and detection of the $h$ should be easier. However, the most unique signature of a non-standard model Higgs boson spectrum is inevitably the existence of charged Higgs bosons. We turn next to a consideration of techniques for their detection.

\section{Charged Higgs scalars}

The structure of the Higgs sector in theories that go beyond the minimal standard model is always such that more than one $\mathrm{SU}(2)_{\mathrm{L}}$ doublet of complex scalar fields is introduced. In this case, not all of the charged Goldstone bosons can become the longitudinal modes of massive charged gauge bosons. Inevitably, one or more charged Higgs bosons emerge. In the most common case of two doublets, a charged pair, $\mathbf{H}^{ \pm}$, exists. Thus knowing the spectrum of fundamental charged scalars is very important, and the mere existence of a charged scalar provides an incontrovertible signature for structure beyond the SM.

SLC and LEP will surely observe any charged scalars lighter than about $40 \mathrm{GeV}$; they will be pair produced and their primary decays to the heaviest allowed charged fermion anti-fermion decay mode will be detectable since backgrounds from random jets are not too large [45]. If the t-quark is observed at Tevatron-I in the usual semi-leptonic decay modes, we will know that $m_{\mathrm{H}^{ \pm}}>m_{\mathrm{t}}-m_{\mathrm{b}}$. Otherwise the $\mathrm{t}$ would have decayed semi-weakly via $\mathrm{t} \rightarrow \mathrm{bH}^{+}$, followed by $\mathrm{H}^{+} \rightarrow \mathrm{cb}$ or $\mathrm{cs}$ or $\tau^{+} \nu_{\tau}$, 
but not to lighter modes with $\mu \nu_{\mu}$ or $\mathrm{e} \nu_{\mathrm{e}}$. Conversely, if a $t$ is not observed in the usual ways, there is a possibility that $\mathrm{t} \rightarrow \mathrm{H}^{+} \mathrm{b}$, which is difficult to observe directly. The present lower limits on $m_{\mathrm{H}^{*}}$ are about $19 \mathrm{GeV}$, most completely analyzed recently by the CELLO group [46]. That implies $m_{\mathrm{t}}>24 \mathrm{GeV}$.

\subsection{BRANCHING RATIOS}

The $\mathrm{H}^{ \pm}$branching ratios are necessarily model dependent. When two or more doublets are present, the couplings of the $\mathrm{H}^{ \pm}$to fermions and to gauge bosons are not unique. This is illustrated by the coupling of the $\mathrm{H}^{+}$to fermions and gauge bosons in the minimal supersymmetric model, see refs. [24] and [47]. Its coupling to a $u \bar{d}$ channel ( $u$ is any up quark and $d$ its down partner) is given by

$$
\frac{i g}{\sqrt{2} m_{\mathrm{W}}}\left[m_{\mathrm{d}} \tan \beta P_{\mathrm{L}}+m_{\mathrm{u}} \cot \beta P_{\mathrm{R}}\right] \text {, }
$$

where $\tan \beta=v_{2} / v_{1}$ is the ratio of vacuum expectation values for the neutral fields of the two complex doublets. For gauge bosons, the $\mathrm{W}^{+} \mathrm{H}^{-} \mathrm{H}^{0}$ vertex contains a factor

$$
\frac{1}{2} g\left(p+p^{\prime}\right)^{\mu}\left\{\begin{array}{c}
\sin (\alpha-\beta) \\
\text { or } \\
\cos (\alpha-\beta)
\end{array}\right.
$$

for the heavier $(\mathrm{H})$ and the lighter (h) of the two neutral scalars, respectively. Note the sum rule:

$$
g_{\mathrm{H}^{+} \mathrm{W}^{-} \mathrm{h}}^{2}+g_{\mathrm{H}^{+} \mathrm{W}^{-} \mathbf{H}^{2}}^{2}=\frac{1}{4} g^{2}
$$

A specific model is required to determine the relationship between the angles $\alpha$ and $\beta$ and the masses of the $h$ and $H$; the minimal supersymmetry model [24] is one possibility.

Unfortunately, in most theories there is no $\mathrm{W}^{+} \mathrm{H}^{-} \mathrm{Z}$ tree level vertex. The background for the resulting $\mathrm{W}^{+} \mathrm{Z}$ decay mode of the $\mathrm{H}^{+}$, deriving from $\mathrm{u} \overline{\mathrm{d}} \rightarrow \mathrm{W}^{+} \mathrm{Z}$, is such that branching ratios of a few percent would be required in order to see a signal; it is unlikely that one loop contributions could be large enough. Detection of a charged scalar boson decaying to $\mathrm{W}^{ \pm} \mathrm{Z}$ would be a clear proof of a non-standard Higgs mechanism [48], which goes beyond the normal two doublet structure. The sensitivity of the SSC to such a mode, using $\mathrm{W} \pm \rightarrow \ell \pm \nu, Z \rightarrow \ell^{+} \ell^{-}$is large enough to be interesting. 
Barring the above "exotic" mode, the modes for which there exist tree-level couplings of the charged $\mathrm{H}^{+}$are:

$$
\mathrm{H}^{+} \rightarrow\left\{\begin{array}{l}
\overline{\mathrm{tb}} \\
\overline{\mathrm{c}} \mathrm{b} \\
\tau^{+} \nu \\
\mathrm{W}^{+} \mathrm{H}^{0}
\end{array}\right.
$$

at the two-body level, and

$$
\mathrm{H}^{+} \rightarrow\left\{\begin{array}{l}
\mathrm{W}^{+} \gamma \mathrm{H}^{0} \\
\mathrm{~W}^{+} \mathrm{ZH}^{0}
\end{array},\right.
$$

at the three-body level, where $\mathrm{H}^{0}$ can be either the heavy or the light neutral scalar Higgs of the two-doublet model.

For these three-body modes the vertex factors in the case of the lighter scalar Higgs $h$ are

$$
\frac{-i g^{2} \sin ^{2} \theta_{\mathrm{W}}}{2 \cos \theta_{\mathrm{W}}} \cos (\beta-\alpha) g^{\mu \nu}
$$

for the $\mathrm{Z}$ mode, and

$$
-\frac{1}{2} i g e \cos (\beta-\alpha) g^{\mu \nu}
$$

for the $\gamma$ mode. For the heavy Higgs scalar the $\cos (\beta-\alpha)$ factor would be replaced by $\sin (\beta-\alpha)$, i.e. there is a sum rule analogous to eq. (19).

The only one loop mode that appears to be large enough to be detectable is

$$
\mathrm{H}^{ \pm} \rightarrow \mathrm{W}^{ \pm} \gamma
$$

The calculation is described in detail in the appendix, since to our knowledge this decay has not previously been considered.

Assuming that $\overline{t b}$ is the dominant mode, the branching ratio of $\mathrm{H}^{+}$to $\tau^{+} \nu$ is roughly

$$
\mathrm{BR}\left(\mathrm{H}^{ \pm} \rightarrow \tau \nu\right) \approx \frac{m_{r}^{2} \tan ^{2} \beta}{3\left(m_{\mathrm{t}}^{2} \cot ^{2} \beta+m_{\mathrm{b}}^{2} \tan ^{2} \beta\right)}
$$

Unless $\tan \beta$ is quite large, we expect a branching ratio of $\mathrm{BR}\left(\mathrm{H}^{+} \rightarrow \tau \nu\right) \leq 10^{-3}$, when $m_{\mathrm{H}^{+}}>m_{\mathrm{t}}+m_{\mathrm{b}}$ and $m_{\mathrm{t}} \geqslant 55 \mathrm{GeV}$. Of course, if the top quark mass is larger than the charged Higgs mass, then $\mathrm{BR}(\tau \nu)$ can be substantially bigger; at $\tan \beta=1$ roughly $35 \%$ of the charged Higgs decays are to $\tau \nu$, and the number could be substantially higher if $\tan \beta>1$. To be more specific requires a definite model, 
which also includes the $\mathrm{W}^{+} \mathrm{H}^{0}$ modes described earlier. As an example, if the branching ratio for $\mathrm{H}^{+} \rightarrow \tau^{+} \nu$ is computed in the minimal supersymmetry model [34], for $m_{\mathrm{H}^{ \pm}}<m_{\mathrm{t}}+m_{\mathrm{b}}$ and all SUSY particle modes forbidden, a typical choice of parameters leads to $\tan \beta$ values as a function of $m_{\mathrm{H}^{ \pm}}$such that the cis and $\mathrm{W}^{+} \mathrm{H}^{0}$ modes are not large and the $\tau \nu$ mode branching ratio ranges between $10 \%$ and $40 \%$, and even higher for small $m_{\mathrm{H}^{+}}$.

The $\mathrm{H}^{+} \rightarrow \mathrm{W}^{+} \mathrm{h}$ and $\mathrm{H}^{+} \rightarrow \mathrm{W}^{+} \mathrm{H}$ decays are potentially quite important due to the large contributions from longitudinal $\mathrm{W}$ polarization states. Defining the ratio $R_{\mathrm{W}^{+} \mathrm{H}^{0}} \equiv \mathrm{BR}\left(\mathrm{H}^{+} \rightarrow \mathrm{W}^{+} \mathrm{H}^{0}\right) / \mathrm{BR}\left(\mathrm{H}^{+} \rightarrow \mathrm{tb}\right)$, we obtain:

$$
R_{\mathrm{W}^{+} \mathrm{h}}=\frac{2 \cos ^{2}(\beta-\alpha) p_{\mathrm{W}^{3} m_{\mathrm{H}^{+}}^{2}}}{3 p_{\overline{\mathrm{b}}}\left[\left(m_{\mathrm{t}}^{2} \cot ^{2} \beta+m_{\mathrm{b}}^{2} \tan ^{2} \beta\right)\left(m_{\mathrm{H}^{+}}^{2}-m_{\mathrm{t}}^{2}-m_{\mathrm{b}}^{2}\right)-4 m_{\mathrm{t}}^{2} m_{\mathrm{b}}^{2}\right]},
$$

where $p_{\mathrm{W}}$ and $p_{\overline{\mathrm{b}}}$ are the center-of-mass momenta of the indicated final state particles, and $\alpha$ is the scalar Higgs mixing angle. The corresponding formula for $\mathrm{H}$ is obtained by replacing $\cos (\beta-\alpha)$ with $\sin (\beta-\alpha)$. To obtain an idea of just how

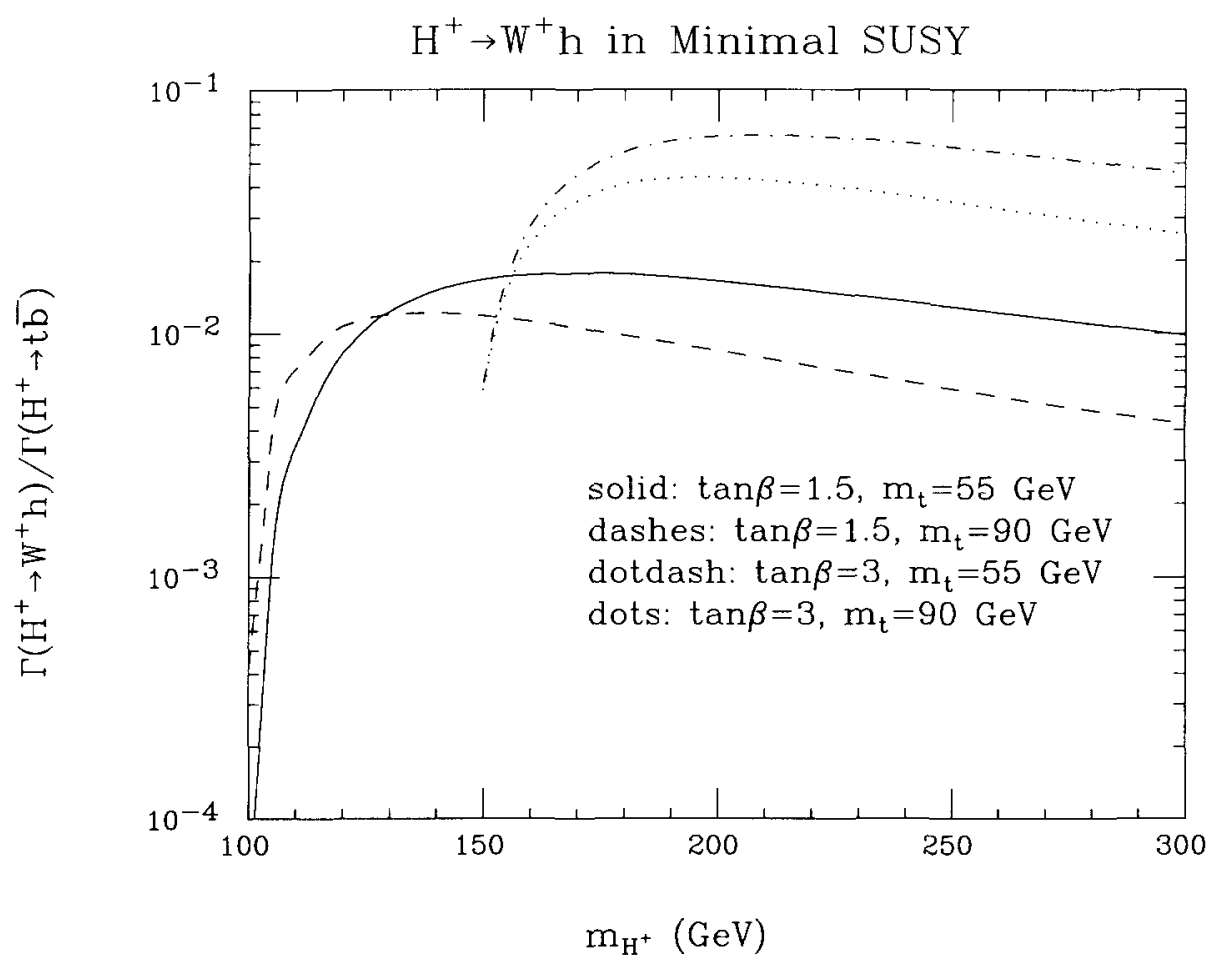

Fig. 11. We plot the ratio $R_{\mathrm{W}^{+} \mathrm{h}}$, see eq. (24), for several values of $\tan \beta$ and $m_{\mathrm{t}}$, in the case of the minimal supersymmetric model. 
important these modes could potentially be, we imagine that there is a model in which the outgoing $\mathrm{h}$ has a mass of $40 \mathrm{GeV}$ and saturates the allowed coupling strength (i.e., $\cos (\beta-\alpha)=1$ in eq. (24)). At $\tan \beta=1$ and $m_{\mathrm{t}}=55 \mathrm{GeV}$ the ratio $R_{\mathrm{W}^{+}{ }_{\mathrm{h}}}$ rises from $\sim 0.17$ at $m_{\mathrm{H}^{ \pm}}=140 \mathrm{GeV}$ to $\sim 1.2$ at $m_{\mathrm{H}^{ \pm}}=200 \mathrm{GeV}$, passing 10 in the vicinity of $m_{\mathrm{H}^{ \pm}}=460 \mathrm{GeV}$. However, if the minimal supersymmetric model is employed, the importance of such modes is greatly reduced. First, the mass relations are such that $\mathrm{H}^{+} \rightarrow \mathrm{W}^{+} \mathrm{H}$ is never allowed. Second, the $\cos (\beta-\alpha)$ factor in $g_{\mathrm{H}^{+} \mathrm{W}^{-} \mathrm{h}}$ causes a severe suppression at larger $m_{\mathrm{H}^{ \pm}}$masses. This is the same suppression that occurs in the HWW and HZZ couplings discussed in refs. [24] and [25]. Results for $R_{\mathrm{W}^{+} \mathrm{h}}$ in the minimal supersymmetric model case appear in fig. 11. For instance, at $m_{\mathrm{t}}=55 \mathrm{GeV}$ the resulting $R_{\mathrm{W}^{+} \mathrm{h}}$ value is 0 at $\tan \beta=1(\cos (\beta-\alpha)$ vanishes at this point), and it reaches a maximum as a function of $m_{\mathrm{H}^{+}}$of $\sim 2 \times 10^{-2}$ at $\tan \beta=1.5$ and $\sim 7 \times 10^{-2}$ at $\tan \beta=3$. As $m_{\mathrm{H}^{+}}$increases beyond the location of the maximum $R_{\mathrm{W}^{+} \mathrm{h}}$ falls slowly. The $\mathrm{h}$ masses implied by the choice of $m_{\mathrm{H}^{+}}$and $\tan \beta$ in the above range are of order 20 to $40 \mathrm{GeV}$. Thus h would decay to $b \bar{b}$.

Next, we present results for the decays $\mathrm{H}^{+} \rightarrow \mathrm{W}^{+} \gamma \mathrm{H}^{0}$. The useful branching ratio relative to the tb decay channel of the $\mathrm{H}^{+}$depends upon the minimum energy allowed for the $\gamma$. Let us adopt a requirement of $E_{\gamma} \geqslant 20 \mathrm{GeV}$ in the $\mathrm{H}^{+}$rest frame; this should provide the good trigger and energy resolution required to reduce backgrounds. The calculation requires including three diagrams: the four point vertex diagram with Feynman coupling as specified earlier, and two diagrams where the photon is emitted from either the $\mathrm{H}^{+}$or the $\mathrm{W}^{+}$leg of the three point $\mathrm{H}^{+} \rightarrow \mathrm{W}^{+} \mathrm{H}^{0}$ vertex. These form a gauge invariant set. The decay width arising from these diagrams in the case of $\mathrm{h}$ can be expressed in terms of the four-momenta $p, q$, and $k$ of the $\mathrm{H}^{+}, \mathrm{W}^{+}$and $\gamma$, respectively, as

$$
\Gamma\left(\mathrm{H}^{+} \rightarrow \mathrm{W}^{+} \gamma \mathrm{h}\right)=\int_{x \geqslant x_{\min }}|\mathscr{M}|^{2} \mathrm{~d} \Phi
$$

where

$$
\begin{aligned}
\mathrm{d} \Phi & =\frac{m_{\mathrm{H}^{+}}}{(4 \pi)^{3}} \frac{y \mathrm{~d} x \mathrm{~d} y}{\sqrt{\mu_{\mathrm{W}}^{2}+y^{2}}}, \\
|\mathscr{M}|^{2} & =\left\{2\left[\frac{p \cdot k}{q \cdot k}-1\right]^{2}-\frac{\lambda^{2} m_{\mathrm{H}^{+}}^{2}}{4 \mu_{\mathrm{W}}}\left[\frac{p}{p \cdot k}-\frac{q}{q \cdot k}\right]^{2}\right\}[g e \cos (\beta-\alpha)]^{2},
\end{aligned}
$$

with $y \equiv|q| / m_{\mathrm{H}^{+}}, \quad x \equiv|\boldsymbol{k}| / m_{\mathrm{H}^{+}}, \lambda=\sqrt{1+\mu_{\mathrm{W}}^{2}+\mu_{\mathrm{h}}^{2}-2 \mu_{\mathrm{W}}-2 \mu_{\mathrm{h}}-2 \mu_{\mathrm{W}} \mu_{\mathrm{h}}}$, and $\mu_{\mathrm{W}} \equiv\left(m_{\mathrm{W}} / m_{\mathrm{H}^{+}}\right)^{2}, \mu_{\mathrm{h}} \equiv\left(m_{\mathrm{h}} / m_{\mathrm{H}^{+}}\right)^{2}$. The corresponding width for a final state $\mathrm{H}$, if phase space allowed, would be obtained by the replacement $\cos (\beta-\alpha) \rightarrow \sin (\beta-$ $\alpha$ ). For the previously employed adhoc choices of $\tan \beta=1, m_{\mathrm{t}}=55 \mathrm{GeV}$, an $\mathrm{h}$ mass of $40 \mathrm{GeV}$, and maximal coupling (i.e. $\cos (\beta-\alpha)=1$ ), the ratio $R_{\mathrm{W}^{+} \gamma \mathrm{h}} \equiv$ 


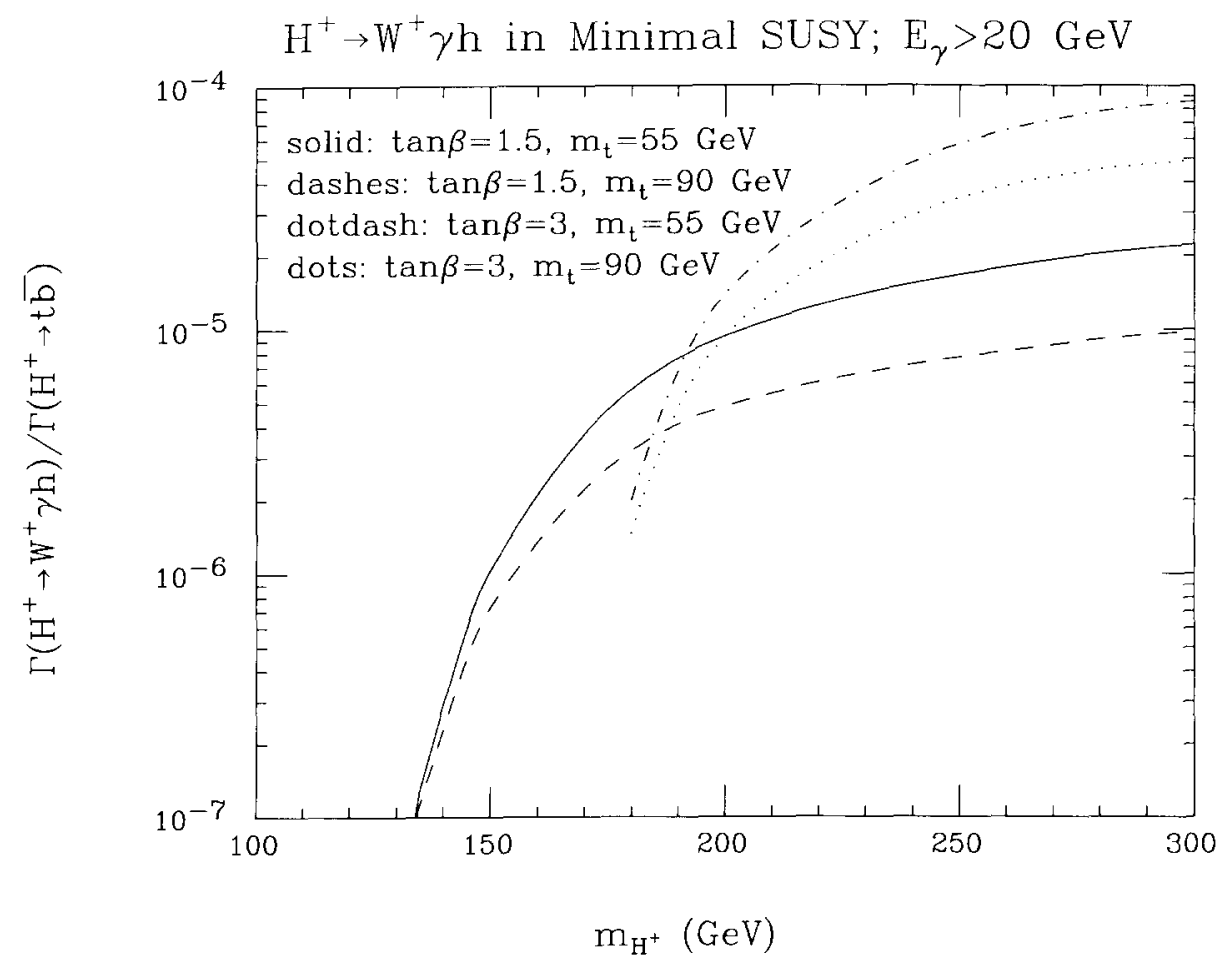

Fig. 12. We plot $R_{\mathrm{W}^{+} \gamma \mathrm{h}} \equiv \mathrm{BR}\left(\mathrm{H}^{+} \rightarrow \mathrm{W}^{+} \gamma \mathrm{h}\right) / \mathrm{BR}\left(\mathrm{H}^{+} \rightarrow \overrightarrow{\mathrm{tb}}\right)$ as a function of $m_{\mathrm{H}^{ \pm}}$for several $m_{\mathrm{t}}, \tan \beta$ values, and $E_{\gamma}>20 \mathrm{GeV}$. We employ the minimal supersymmetric model.

$\mathrm{BR}\left(\mathrm{H}^{+} \rightarrow \mathrm{W}^{+} \gamma \mathrm{h}\right) / \mathrm{BR}\left(\mathrm{H}^{+} \rightarrow \mathrm{tb}\right)$ is 0.01 at $m_{\mathrm{H}^{ \pm}}=300 \mathrm{GeV}$ and rises steadily with increasing $m_{\mathrm{H}^{ \pm}}$, passing 0.1 by $m_{\mathrm{H}^{ \pm}} \sim 520 \mathrm{GeV}$. This would clearly provide a very viable signature (one-sixth of the $\mathrm{W}$ decays are to $\ell^{+} \nu$ ) and rate for $\mathrm{H}^{+}$detection, even accounting for the need to reconstruct the $h$ in a $b \bar{b}$ decay mode. However, just as in the $\mathrm{W}^{+} \mathrm{H}^{0}$ mode case, the minimal supersymmetric model predicts that $\mathrm{H}^{+} \rightarrow \mathrm{W}^{+} \gamma \mathrm{H}$ is phase-space forbidden, while $\mathrm{H}^{+} \rightarrow \mathrm{W}^{+} \gamma \mathrm{h}$ is severely suppressed. Results for $E_{\gamma} \geqslant 20 \mathrm{GeV}$ are given in fig. 12, for several $\tan \beta$ and $m_{\mathrm{t}}$ values. We see that with $E_{\gamma} \geqslant 20 \mathrm{GeV}, m_{\mathrm{t}}=55 \mathrm{GeV}$, and $\tan \beta=1.5$, as an example, this particular model predicts that $R_{\mathrm{W}^{+} \gamma \mathrm{h}}$ reaches a maximum of $\sim 2.6 \times 10^{-5}$ at $m_{\mathrm{H}^{ \pm}} \sim 400 \mathrm{GeV}$ and decreases (slowly) thereafter. The resulting event rate at the SSC would not be useful!

The $\mathrm{W} \gamma$ mode also has a very model dependent branching ratio since the charged and neutral scalars enter the loops, and factors of $\cos (\beta-\alpha)$ and $\tan \beta$ abound. For some ranges of masses of the scalars the $\mathrm{W}_{\gamma}$ branching ratio can be rather large $\left(>10^{-4}\right)$, but in the minimal supersymmetric models there are cancellations and $\mathrm{BR}\left(\mathrm{H}^{+} \rightarrow \mathrm{W}^{+} \gamma\right)$ is rather small, below $10^{-5}$ after the $\mathrm{W}^{+} \rightarrow \ell^{+} \boldsymbol{p}$ branching ratio of 
$\sim \frac{1}{6}$ is included. To give a few specific numbers, we quote results in the case of the minimal supersymmetric model with $m_{\mathrm{t}}=40 \mathrm{GeV}, m_{\mathrm{h}}=0.5 m_{\mathrm{Z}}\left(m_{\mathrm{H}}\right.$ is determined by the value chosen for $m_{\mathrm{H}^{ \pm}}$) and a light neutralino-chargino sector. We find:

$$
\operatorname{BR}\left(\mathrm{H}^{+} \rightarrow \mathrm{W}^{+} \gamma\right)= \begin{cases}7.6 \times 10^{-6}: & m_{\mathrm{H}^{ \pm}}=116 \mathrm{GeV} \\ 2.0 \times 10^{-5}: & m_{\mathrm{H}^{ \pm}}=160 \mathrm{GeV} \\ 2.8 \times 10^{-5}: & m_{\mathrm{H}^{ \pm}}=200 \mathrm{GeV}\end{cases}
$$

where the $\mathrm{W}$ decay branching ratio has not yet been included.

Finally we mention the possibility of observing the $\mathrm{H}^{ \pm}$in its $\mathrm{W} \pm \Upsilon$ or $\mathrm{W} \pm \Theta$ decay modes. These were considered in ref. [49], and we briefly summarize the conclusions. If $\mathbf{H}^{+} \rightarrow \overline{t b}$ is not allowed, then the branching ratio for $\mathrm{H}^{+} \rightarrow \mathrm{W}^{+} \Upsilon$ is quite significant (typically $1-3 \times 10^{-2} \mathrm{BR}\left(\mathrm{H}^{+} \rightarrow \tau^{+} \nu\right)$ ) when $m_{\mathrm{H}^{ \pm}}$is just below $m_{\mathrm{t}}+m_{\mathrm{b}}$, although it falls rapidly with increasing $m_{\mathrm{t}}$. In contrast, since $\mathrm{H}^{+} \rightarrow \mathrm{tb}$ is always allowed if $\mathrm{H}^{+} \rightarrow \mathrm{W}^{+} \Theta$ is, the latter decay inevitably has a very small branching ratio (typically $\leq 10^{-5}$ ), and is not useful.

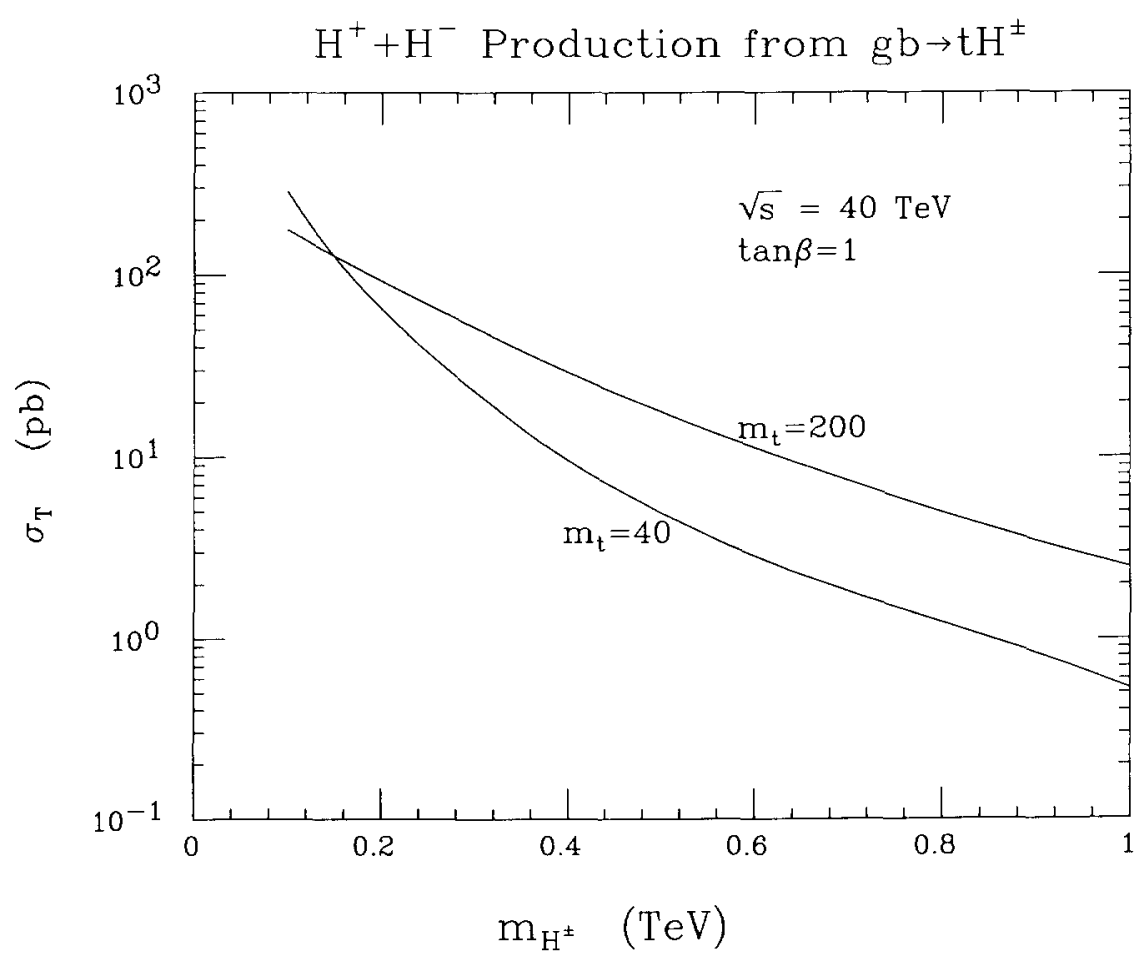

Fig. 13. The $\mathrm{H}^{ \pm}$cross sections from ref. [34]. 


\subsection{PRODUCTION CROSS SECTION}

The primary production mode of the $\mathrm{H}^{ \pm}$depends strongly upon the top quark mass. If the top quark has a moderate mass, but $m_{\mathrm{t}}>m_{\mathrm{H}^{+}}+m_{\mathrm{b}}$, then the rate for $\mathrm{gg} \rightarrow \mathrm{t} \overline{\mathrm{t}}$ followed by $\mathrm{t}$ decay to the $\mathrm{H}^{ \pm}$is very large. Relative to the $\mathrm{t}$ decay rate to charged W's we have:

$$
\frac{\Gamma\left(\mathrm{t} \rightarrow \mathrm{H}^{+} \mathrm{b}\right)}{\Gamma\left(\mathrm{t} \rightarrow \mathrm{W}^{+} \mathrm{b}\right)}=\frac{p_{\mathrm{H}^{+}}}{p_{\mathrm{W}^{+}}} \frac{m_{\mathrm{t}}^{2}\left(m_{\mathrm{t}}^{2}-m_{\mathrm{H}^{+}}^{2}\right)}{\left(m_{\mathrm{t}}^{2}+2 m_{\mathrm{W}}^{2}\right)\left(m_{\mathrm{t}}^{2}-m_{\mathrm{W}}^{2}\right)} \cot ^{2} \beta,
$$

where $p_{\mathrm{H}^{+}}$and $p_{\mathrm{W}^{+}}$are the center-of-mass momenta of the $\mathrm{H}^{+}$and $\mathrm{W}^{+}$for the respective decays. Thus, the $\mathrm{H}^{+}$channel is fully competitive with the $\mathrm{W}^{+}$mode.

When $m_{\mathrm{t}} \leqslant m_{\mathrm{H}^{ \pm}}+m_{\mathrm{b}}$ the cross section for $\mathrm{H}^{ \pm}$production is entirely due to tb fusion processes. A careful calculation, including $m_{t}$ dependence was performed in ref. [34]. There it was found that an excellent approximation to the full QCD computation (including evolution,...) for, say, $\mathrm{H}^{+}$is obtained by computing the $2 \rightarrow 3$ subprocess $\mathrm{g} \overline{\mathrm{b}} \rightarrow \overline{\mathrm{t}} \mathrm{H}^{+}$, retaining $m_{\mathrm{t}}$ in both the amplitudes and the kinematics. (Note, for future reference, that the $\mathrm{H}^{+}$is produced in association with a spectator $\overline{\mathrm{t}}$.) The rates are large, with over $10^{6} \mathrm{H}^{ \pm}$being produced for $m_{\mathrm{H}^{+}} \leqslant 200$ $\mathrm{GeV}$ at the SSC. The cross section, for several $m_{\mathrm{t}}$ values, is shown in fig. 13, where we have taken $\tan \beta=1$ in the $\mathrm{H}^{+}$tb coupling given earlier.

\subsection{BACKGROUNDS}

The question of whether or not a charged Higgs could be seen in its tb decay mode remains unanswered. A first survey of backgrounds is being done in ref. [50]. The irreducible SM background for the $\overline{\mathrm{t}} \overline{\mathrm{b}}$ decay mode of the $\mathrm{H}^{+}$is not nearly so large as the $\bar{t}$ mode was for the SUSY h or SM H $\mathrm{SM}_{\mathrm{SM}}^{0}$, because there are fewer ways to produce $\overline{\mathrm{b}} \overline{\mathrm{b}}$. Either a $\mathrm{W}^{+}$is involved, and hence a factor of $G_{\mathrm{F}}$, or $\mathrm{t} / \mathrm{b}$-quark distributions in a proton, which are not large. Thus, if a $\bar{t} \bar{b}$ channel can be flavor tagged, by discriminating between it and other two-jet channels, with good efficiency, there might be a chance to detect the $\mathrm{H}^{+}$in this channel over some mass range. We will sketch the results of ref. [50] for the typical case of $m_{\mathrm{H}^{ \pm}}=100 \mathrm{GeV}$ and $\tan \beta=1$. First, we require that all outgoing jets have $|y|<5$ and energies above $10 \mathrm{GeV}$. In addition, we require that the $\mathrm{b}$ jet (or $\mathrm{g}$ ) have energy larger than $50 \mathrm{GeV}$, and that the laboratory angular separation between the $\overline{\mathrm{t}}$ and $\mathrm{b}$ (or $\mathrm{g}$ ) be at least 15 degrees. Finally, we assume that it will be possible to achieve a resolution of order $10 \%$ in the $\overline{\mathrm{t}}-\mathrm{b}(\mathrm{g})$ mass, and accept only events with $M_{\mathrm{tb}(\mathrm{g})}$ within the range 95 $\mathrm{GeV}$ to $105 \mathrm{GeV}$. The results for some of the relevant cross sections are (taking $\tan \beta=1$ for the $\mathrm{H}^{ \pm}$coupling to tb):

$$
\begin{gathered}
\sigma\left(\mathrm{t}+\mathrm{H}^{-}\right)-64 \mathrm{pb}, \\
\sigma(\mathrm{t}+\overline{\mathrm{tb}})-200 \mathrm{pb}, \\
\sigma(\mathrm{t}+\overline{\mathrm{tg}})-2600 \mathrm{pb},
\end{gathered}
$$


the latter two being initiated by $\mathrm{gb}$ and gg collisions, respectively. It is relatively straightforward to find additional cuts that reduce the $\mathrm{t}+\overline{\mathrm{t}} \mathrm{b}$ background below the signal rate (without sacrifice of too much cross section). Thus the most important question is whether efficient procedures for distinguishing $b$ jets from $g$ jets to one part in 40 can be developed. Of course, we have so far ignored backgrounds such as gg $\rightarrow$ ggg (and similar ones involving light quarks), which enter to the extent that $t$ jets cannot be distinguished from $g$ and light quark jets, and $g b \rightarrow b \bar{b} b$, which enters if $a \mathrm{~b}$ quark jet cannot be separated from a $\mathrm{t}$ jet. Once $m_{\mathrm{t}}$ is known it may be possible to develop strategies for $t$ quark jet triggering that will enable us to sufficiently discriminate against all such backgrounds. For instance, the t-spectator trigger discussed below and in ref. [34] should be effective in reducing a large class of backgrounds. In addition, it is likely that one must use semi-leptonic decays and $p_{\mathrm{T}}$ cuts relative to jet axes, for the $\mathrm{H}^{+}$decay products themselves. Ultimately, only careful Monte Carlo studies can give a reliable assessment of the viability of this mode. We note, for this reason, that it is important to have $\bar{t} \bar{b}$ production in the Monte Carlo programs.

Triggering on the $\tau \nu$ mode of $\mathrm{H}^{ \pm}$decay probably implies looking for the single particle decay modes of the $\tau$. Of course, a vertex detector would also be enormously helpful. The primary background to the $\tau \nu$ decay mode of the $\mathrm{H}^{ \pm}$, discussed in ref. [34], was $\mathrm{W}$ or $\mathrm{W}^{*}$ production, followed by $\mathrm{W}$ decay to $\tau \nu$; it was found to be serious. However, it was pointed out there that the $\mathrm{H}^{+}$is always produced in association with a $\overline{\mathrm{t}}$ in the beam or target spectator system, and the triggering on this associated heavy top quark (via its leptonic decay products) provides an enormous suppression of backgrounds. Even so the viability of the $\tau^{+} \nu$ mode was not completely obvious. In particular, the process $\mathrm{g} \bar{b} \rightarrow \overline{\mathrm{t}} \mathrm{W}^{+}$is not suppressed by the spectator t trigger. Additional backgrounds to $\tau^{+} \nu$ come from Drell-Yan $\tau^{+} \tau^{-}$ production and $\mathrm{W}$ pair production (where at least one of the W's decays to $\tau^{+} \nu$ ), and heavy quark decays. As part of the Madison 1987 workshop a serious Monte Carlo study of this mode was undertaken [51]. This study was continued at the 1987 LBL workshop [52]. Generally the conclusions are rather pessimistic until the branching ratio to the $\tau \nu$ mode exceeds roughly $50 \%$, a range that is not terribly likely in a typical SUSY model unless $h$ is very near in mass to the $Z$ (assuming as stated earlier that the $\bar{t} \bar{b}$ decay channel is open).

The background to $\mathrm{H}^{ \pm} \rightarrow \mathrm{W}^{ \pm} \gamma$ coming from $\mathrm{q} \overline{\mathrm{q}} \rightarrow \mathrm{W}{ }^{ \pm} \gamma$ is not quite as large as that for the related neutral Higgs decay modes, $Z \gamma$ and $\gamma \gamma$, due to the well-known radiation zeros in the cross section [53] as a function of angle. The signal to background ratio can be enhanced by retaining only events in which the $\gamma$ emerges at large angles with respect to the beam direction (in the $\mathrm{W}^{ \pm} \gamma$ center of mass). Equivalently, a cut requiring small rapidities for the $\gamma$ is very effective. Compare for example the distributions of figs. 137 and 140 in ref. [54]. Of course, a spectator $t$ quark trigger will again be very effective in removing this background, since very few $\mathrm{W}^{ \pm} \gamma$ continuum events will have an associated top quark. 
The backgrounds coming from mixed $\mathrm{QCD} /$ electroweak processes are likely to be substantial in the $\mathrm{W}^{ \pm} \mathrm{b} \overline{\mathrm{b}}$ final state arising from $\mathrm{H}^{ \pm} \rightarrow \mathrm{W}^{ \pm} \mathrm{H}^{0}$ followed by $\mathrm{H}^{0} \rightarrow b \bar{b}$. In fact, it is these same backgrounds which create difficulty for the associated $\mathrm{W}+\mathrm{H}_{\mathrm{SM}}^{0}$ production technique for finding the SM neutral Higgs. For example, consider the minimal SUSY model $h$. The branching ratios given earlier, e.g. for $\tan \beta \geqslant 1.5$, imply an effective $\mathrm{W}^{+} \mathrm{h}$ associated production cross section ( $\sim 1$ $\mathrm{pb}$ ) that is not very different from the associated production cross section [55]. (Of course, outside the context of the minimal SUSY model considerably larger cross sections are possible.) As we have discussed earlier, these studies of the intermediate mass Higgs region [55] suggest that the $b \bar{b}$ mass resolution will be sufficient to recognize a $1 \mathrm{pb}$ level $\mathrm{W}^{+} \mathrm{H}_{\mathrm{SM}}^{0}$ signal over backgrounds coming from mixed $\mathrm{QCD} /$ electroweak processes such as $\mathrm{q} \bar{q}^{\prime} \rightarrow \mathrm{W}^{+} \mathrm{b} \overline{\mathrm{b}}$, when the $\mathrm{H}_{\mathrm{SM}}^{0}$ mass is in the vicinity of $120 \mathrm{GeV}$ (and the $\mathrm{H}_{\mathrm{SM}}^{0} \rightarrow \mathrm{tt}$ mode is not allowed). This would undoubtedly be much more difficult at lower $\bar{b} \bar{b}$ invariant mass; in particular, the backgrounds would be significantly larger. On the other hand, a charged Higgs decay would lead to jacobian peaks in the outgoing $\mathrm{W}^{+}$and $b \bar{b}$-system transverse momenta that might allow cuts that would compensate for this increase in background. And, in addition, triggering on the $\mathrm{t}$ quark spectator to $\mathrm{H}^{+}$production, as in previous cases, will yield a very substantial suppression of continuum $W^{+} b \bar{b}$ backgrounds. Clearly, a detailed Monte Carlo study is required to fully assess the situation. Similarly, the $\mathrm{W}^{ \pm} \mathrm{H}^{0} \gamma$ and $\mathrm{W}^{ \pm} \mathrm{H}^{0} \mathrm{Z}$ modes require complicated QCD estimates and detailed Monte Carlo studies. Without such studies it is impossible to judge whether $\mathrm{H}^{ \pm}$detection in these modes is possible. Certainly, as we have noted earlier, in the minimal SUSY model the rates would not be useful.

Finally, with regard to the decay $\mathrm{H}^{ \pm} \rightarrow \mathrm{W}{ }^{ \pm} r$, we note that for $\bar{t}$ production, followed by $\mathrm{t} \rightarrow \mathrm{H}^{+} \mathrm{b}$ and $\overline{\mathrm{t}} \rightarrow \mathrm{W}^{-} \overline{\mathrm{b}}$, one finds a significant rate for production of two $b$ jets, two leptonically decaying $W$ 's and a leptonically decaying $r$. Backgrounds should be quite small.

\subsection{RESULTS}

The intrinsic model dependence of the $\mathrm{H}^{ \pm}$branching ratios, and the present lack of Monte Carlo studies of crucial backgrounds, combine to make definitive statements on $\mathbf{H}^{ \pm}$detection not possible. The situation is by no means hopeless. The production cross sections are large. A number of modes exist with possible signatures if certain detector capabilities are available. The likely dominant decay, $t \bar{b}$, could turn out to be useful depending upon the effectiveness of flavor tagging (using, for instance, the leptons in the $t$ and $b$ jets) and of heavy quark spectator triggering. A study of irreducible backgrounds is underway. At larger $m_{\mathrm{H}^{ \pm}}$, several modes, such as $\mathrm{W}^{ \pm} \gamma, \mathrm{W}^{ \pm} \mathrm{h}, \mathrm{W}^{ \pm} \gamma \mathrm{h}$, and $\mathrm{W}{ }^{ \pm} \mathrm{Zh}$, may be available; the latter three occur at tree level. In certain regions of parameter space the $\mathrm{H}^{ \pm} \rightarrow \tau \nu$ branching ratio is $\gtrsim 10^{-2}$, and the $\tau \nu$ channel would provide a means for detection of the charged Higgs. 
General studies of $\mathrm{H}^{ \pm}$detection should presumably proceed by first learning what the backgrounds are, and then finding the ranges of parameters for which a signal could be detected. At $\mathrm{e}^{+} \mathrm{e}^{-}$colliders, where the $\overline{\mathrm{tb}}$ modes can clearly be employed, the decreasing cross section and the limitations of flavor tagging will set the limit. Studies suggest that, when the $\mathrm{H}^{+} \mathrm{H}^{-}$pair mass is not too near $\sqrt{s}$, detection will indeed be feasible. Because of the great importance of eventually measuring the spectrum of charged scalar bosons, it should be a high priority to continue these efforts.

\section{Comments and conclusions}

We have demonstrated that, possibly apart from one small region in $m_{\mathrm{t}}-m_{\mathrm{H}_{\mathrm{SM}}^{0}}$ space, detection of a neutral intermediate mass SM Higgs boson should be feasible at a high energy hadron collider. In supersymmetric theories, and other models that go beyond the standard model, the detectability of an intermediate mass neutral Higgs boson is dependent upon many new considerations. Detection could become easier since production can be enhanced and additional detection modes can become available. However, if only SM particle decay modes are phase-space allowed, some difficulties also arise. For instance, among the various neutral Higgs in the minimal two-doublet supersymmetric theory, the $\mathbf{h}$ has mass below $m_{Z}$ and could fall below the region accessible via the $\gamma \gamma$ decay mode, while the $\mathrm{H}$ starts to decouple from the $\mathrm{ZZ}^{*}$ channel as its mass approaches $2 m_{\mathrm{Z}}$.

In comparing the SSC to the $\mathrm{LHC}$, it is interesting to note that even though we are discussing particles of order $100 \mathrm{GeV}$ in mass, the Higgs production cross section is lower by about a factor of five if $\sqrt{s}$ is reduced from $40 \mathrm{TeV}$ to $17 \mathrm{TeV}$. This is shown in fig. 5, and agrees with the decreased gluon luminosity found in the studies performed in ref. [56]. Since gg fusion is the main production mechanism for $\mathbf{H}_{S M}^{0}$, the $\mathrm{H}_{\mathrm{SM}}^{0}$ cross section is correspondingly reduced. It is also noteworthy that most backgrounds do not decrease with $\sqrt{s}$ as rapidly, so that signal/background becomes worse at the same time as the signal decreases.

For charged scalars the intrinsic model dependence of the couplings and the lack of work on simulations of backgrounds make it more difficult to draw conclusions. The situation is not necessarily bad, since production cross sections at a hadron collider are large, and a variety of modes with reasonable signatures exist. The fact that the charged Higgs is inclusively produced in association with a top quark, which can be triggered on, will undoubtedly prove enormously helpful.

We have largely restricted ourselves to standard particles for decay channels and loops. The effect of supersymmetric partners in the loops for $h \rightarrow \gamma \gamma$ has been studied [29], and the calculation we report for $\mathrm{H}^{+} \rightarrow \mathrm{W}^{+} \gamma$ contains the full contribution from two Higgs doublets and supersymmetric partner loops. We have also given branching ratios for $\mathrm{H}^{+} \rightarrow \mathrm{W}^{+} \mathrm{h}$ and $\mathrm{H}^{+} \rightarrow \mathrm{W}^{+} \gamma \mathrm{h}$ within the context of the minimal two-doublet supersymmetry model. Further study of branching ratios in 
extensions of the standard model would be useful, and have been begun [57]. We have also not considered decay modes into supersymmetric partners or other new particles. Indeed, the work of refs. [25], [34] and [57] demonstrates that, when allowed, decay of all the two-doublet model SUSY Higgs, h, H, A and $\mathbf{H}^{ \pm}$, to channels containing charginos and neutralinos will dominate. While the phase-space for this in the case of the $h$ (with mass $\leqslant m_{Z}$ ), or a low-mass $\mathrm{H}^{ \pm}$, is not very large, such modes will be very important for the SUSY model heavy neutral Higgs and for the $\mathrm{H}^{ \pm}$(when it is heavy). A sample study for a typical heavy neutral SUSY Higgs was performed in ref. [58], with the conclusion that detection would be straightforward in a mode containing the LSP and the next-lightest neutralino. Such a mode typically has a large branching ratio and has small backgrounds when the next-lightest neutralino decays to photon + LSP. Similarly, there are large branching ratios for $\mathrm{H}^{ \pm}$decay to one chargino and one neutralino, once such modes are phase-space allowed. Signatures appear to be promising [57]. A hard isolated photon, one or more hard charged leptons, and missing energy are typical of the ultimate final state for such a decay. In general, we anticipate that any particular model in which such decays occur will provide straightforward ways to detect the $\mathrm{H}, \mathrm{A}$ and $\mathrm{H}^{ \pm}$.

We wish to conclude by emphasizing the following points.

(i) It is essential to learn how to probe the spectrum of Higgs bosons in the intermediate mass region. In particular, we have learned that sensitivity to this sector is not automatic; it depends on machine parameters and detector capabilities, as well as on the theory of SU(2) breaking.

(ii) Electron-positron colliders in the region of a few hundred $\mathrm{GeV}$ and with luminosity above an energy-dependent critical value can probably do the necessary studies, though some further analysis is necessary to cover all the possible scenarios for the $\mathrm{H}^{ \pm}$.

(iii) High energy, high luminosity hadron colliders can probably cover the entire intermediate mass region for the $\mathrm{H}_{\mathrm{SM}}^{0}$, though a little further study of backgrounds is needed to confirm this in some small regions of the $m_{t}-m_{\mathrm{H}_{\mathrm{S}}^{0}}$ plane. To do so detectors must be able to identify isolated $\gamma$ 's, distinguish them from jets to a few parts in $10^{5}$, have better than about $2 \%$ resolution in $\gamma \gamma$ mass, identify a significant fraction of b-quark jets by the $p_{\mathrm{T}}$ of the lepton in the jet, and so on. In addition, detailed event rate studies at the SSC show that the exploration of the intermediate mass region would be greatly simplified by a high luminosity interaction region (with $\sim 10$ times the design luminosity) that focuses on electromagnetic showers with some hadronic energy vetoing.

(iv) For an $\mathrm{H}^{ \pm}$the situation at hadron colliders is unclear. Production cross sections are large, and decays with useful signatures exist. However, theories that go beyond the standard model in such a way as to contain a charged Higgs inevitably introduce substantial model dependence for its couplings and, hence, its decay branching ratios. In addition, there currently are relatively few simulations of the relevant backgrounds to the many different possible detection channels. A channel- 
by-channel survey of the branching ratio that would make charged Higgs detection in a given mode possible is needed.

We would like to acknowledge helpful conversations with D. Atwood, F. Paige, L. Price, and M. Soldate, and assistance from J. Lorenzo Diaz-Cruz. We would also like to thank the University of Wisconsin for its hospitality during the workshop on "From Colliders to Supercolliders" where a portion of this work was performed. This work was supported, in part, by the Department of Energy.

\section{Appendix}

In this appendix we give a review of the $\mathrm{H}_{\mathrm{SM}}^{0} \rightarrow \mathrm{Z} \gamma$ calculation in the standard model [30], and we present the details of the $\mathrm{H}^{+} \rightarrow \mathrm{W}^{+} \gamma$ decay in the minimal supersymmetric model of refs. [47], [24], and [25].

The $\mathrm{H}_{\mathrm{SM}}^{0} \rightarrow \mathrm{Z} \gamma$ decay proceeds, in the unitary gauge of the SM, through $\mathrm{W}$ loops and fermion loops. Aside from its usefulness in the hunt for the intermediate mass Higgs, this mode is interesting in that it is sensitive to the WWZ vertex (and to the $W W Z \gamma$ vertex by the requirement of gauge invariance). The width of the Higgs in this mode is given by [30]

$$
\begin{aligned}
\Gamma\left(\mathrm{H}_{\mathrm{SM}}^{0} \rightarrow \mathrm{Z} \gamma\right) & =\frac{1}{32 \pi}|\mathscr{A}|^{2} m_{0}^{3}\left(1-\frac{m_{\mathrm{Z}}^{2}}{m_{0}^{2}}\right)^{3}, \\
\mathscr{A} & =\frac{e g^{2}}{(4 \pi)^{2} m_{\mathrm{W}}}\left(A_{\mathrm{F}}+A_{\mathrm{W}}\right), \\
A_{\mathrm{F}} & =\sum_{\mathrm{f}} \frac{-2 Q_{\mathrm{f}}\left(T_{\mathrm{f}}^{3 \mathrm{~L}}-2 Q_{\mathrm{f}} s_{\mathrm{W}}^{2}\right)}{c_{\mathrm{W}}}\left[4 I_{1}\left(\frac{m_{\mathrm{Z}}^{2}}{m_{\mathrm{f}}^{2}}, \frac{m_{0}^{2}}{m_{\mathrm{f}}^{2}}\right)-I_{2}\left(\frac{m_{\mathrm{Z}}^{2}}{m_{\mathrm{f}}^{2}}, \frac{m_{0}^{2}}{m_{\mathrm{f}}^{2}}\right)\right], \\
A_{\mathrm{W}} & =-4 c_{\mathrm{W}} m_{\mathrm{W}}^{2}\left\{\left(3-t_{\mathrm{W}}^{2}\right) I_{2}\left(\frac{m_{\mathrm{Z}}^{2}}{m_{\mathrm{W}}^{2}}, \frac{m_{0}^{2}}{m_{\mathrm{W}}^{2}}\right)\right. \\
& \left.\quad+\left[\left(1+\frac{m_{0}^{2}}{2 m_{\mathrm{W}}^{2}}\right) t_{\mathrm{W}}^{2}-\left(5+\frac{m_{0}^{2}}{2 m_{\mathrm{W}}^{2}}\right)\right] I_{1}\left(\frac{m_{\mathrm{Z}}^{2}}{m_{\mathrm{W}}^{2}}, \frac{m_{0}^{2}}{m_{\mathrm{W}}^{2}}\right)\right\},
\end{aligned}
$$

where $m_{0}$ is the $\mathrm{H}_{\mathrm{SM}}^{0}$ mass, $m_{\mathrm{f}}$ is the mass of the fermion in a given fermion loop, $s_{\mathrm{W}}=\sin \left(\theta_{\mathrm{W}}\right)$, etc., and $A_{\mathrm{F}}, A_{\mathrm{W}}$ are, respectively the contributions from the fermion and $\mathrm{W}$ loops. The parametric integrals have been performed explicitly with 
the result

$$
\begin{aligned}
& I_{1}(a, b)=\frac{1}{2} \frac{1}{(a-b)}+\frac{2}{(a-b)^{2}}[f(1 / b)-f(1 / a)] \\
& +\frac{a}{(a-b)^{2}}[g(1 / b)-g(1 / a)], \\
& I_{2}(a, b)=-\frac{2}{(a-b)}[f(1 / b)-f(1 / a)], \\
& f(\lambda)= \begin{cases}-\left[\sin ^{-1}(1 / 2 \sqrt{\lambda})\right]^{2}, & \text { if } 4 \lambda>1 \\
\frac{1}{4}\left[\ln \left(\eta_{+} / \eta_{-}\right)+i \pi\right]^{2}, & \text { if } 4 \lambda<1\end{cases} \\
& g(\lambda)= \begin{cases}\sqrt{4 \lambda-1} \sin ^{-1}(1 / 2 \sqrt{\lambda}), & \text { if } 4 \lambda>1 \\
\frac{1}{2} \sqrt{1-4 \lambda}\left[\ln \left(\eta_{+} / \eta_{-}\right)+i \pi\right], & \text { if } 4 \lambda<1\end{cases} \\
& \eta_{ \pm} \equiv \frac{1}{2}(1 \pm \sqrt{1-4 \lambda})
\end{aligned}
$$

The value of $A_{\mathrm{F}}$ is small compared to $A_{\mathrm{W}}$; even as $m_{\mathrm{f}} \rightarrow \infty$ we always have $A_{\mathrm{F}} \leqslant 0.12$, and for this reason it is usually neglected. It must be kept in mind, however, that if several very heavy fermions exist, their contributions need not be ignorable.

Now let us turn to the more complicated case of the $\mathrm{H}^{+} \rightarrow \mathrm{W}^{+} \gamma$ decay. In the minimal supersymmetric model [24], this decay proceeds through the one loop diagrams shown in fig. 14. There are five classes of diagrams, each one gauge invariant. Aside from the complications due to the increased number of diagrams, there is qualitative difference between this calculation and the one considered above. In the $\mathrm{H}_{\mathrm{SM}}^{0} \rightarrow \mathrm{Z} \gamma$ case there appears only one form factor in the calculation of the amplitude which has the Lorentz structure $k_{\mu} q_{\nu}-k \cdot q g_{\mu \nu}$, where $k$ is the photon's momentum and $q$ the Z's. In the reaction $\mathrm{H}^{+} \rightarrow \mathrm{W}^{+} \gamma$ there are two form factors, the second one being proportional to $\varepsilon_{\mu \nu \alpha \beta} k^{\alpha} q^{\beta}$ which, in contrast with the first case, is not disallowed by $\mathrm{C}$ conservation. This last point deserves a short comment; using $\mathrm{C}$ conservation arguments to restrict the possible form factors might be puzzling, since $\mathrm{C}$ is not a conserved quantum number in either of the above models. The argument for the $\mathrm{H}_{\mathrm{SM}}^{0} \rightarrow \mathrm{Z} \gamma$ case goes through since there is only 

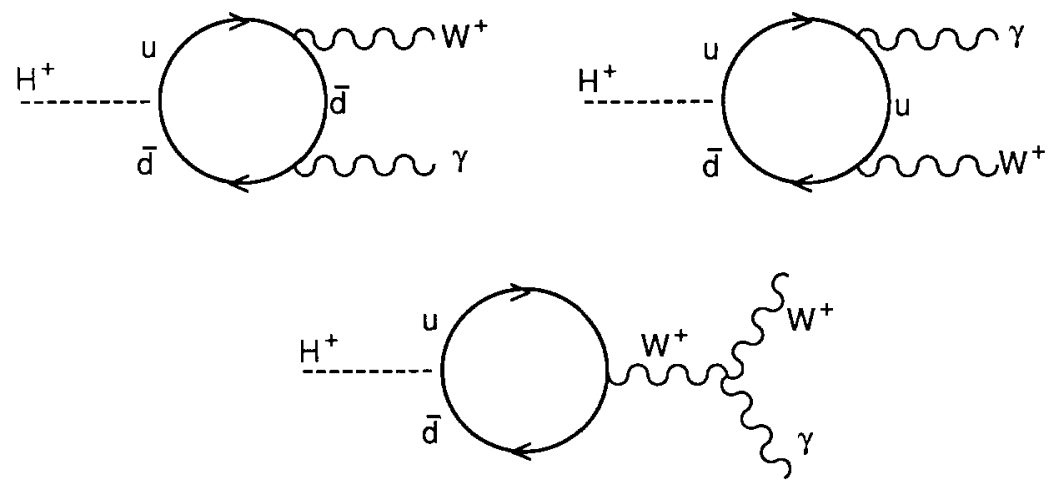

(a)
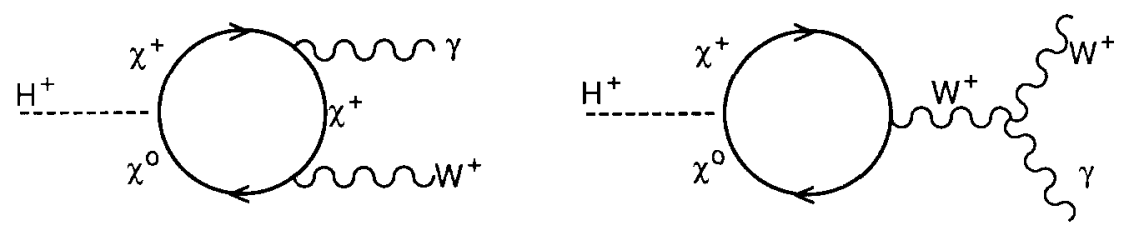

(b)
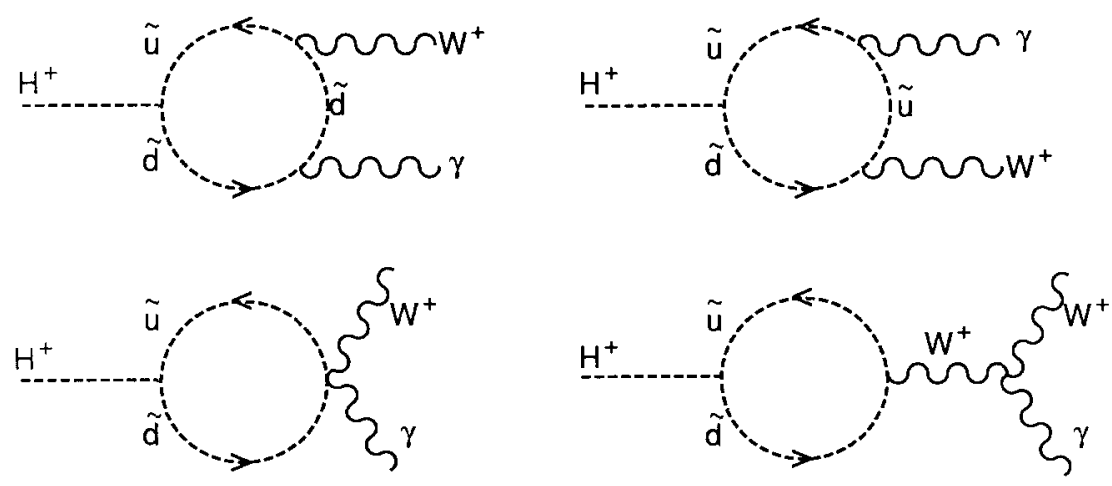

(c)

Fig. 14. The Feynman diagrams for $\mathrm{H}^{ \pm} \rightarrow \mathrm{W}^{ \pm} \gamma$ (in the minimal supersymmetric model of refs. [24] and [25]). (a) Contribution from one fermion doublet. (b) Contribution from charginos and neutralinos. (There are several such particles; corresponding indices have been omitted.) (c) Contribution from a scalar-quark doublet. (d) Contribution from scalar particles. (e) Contribution from W-Higgs loops. (In (d) and (e) there are two contributing scalars whose index is not displayed.) 

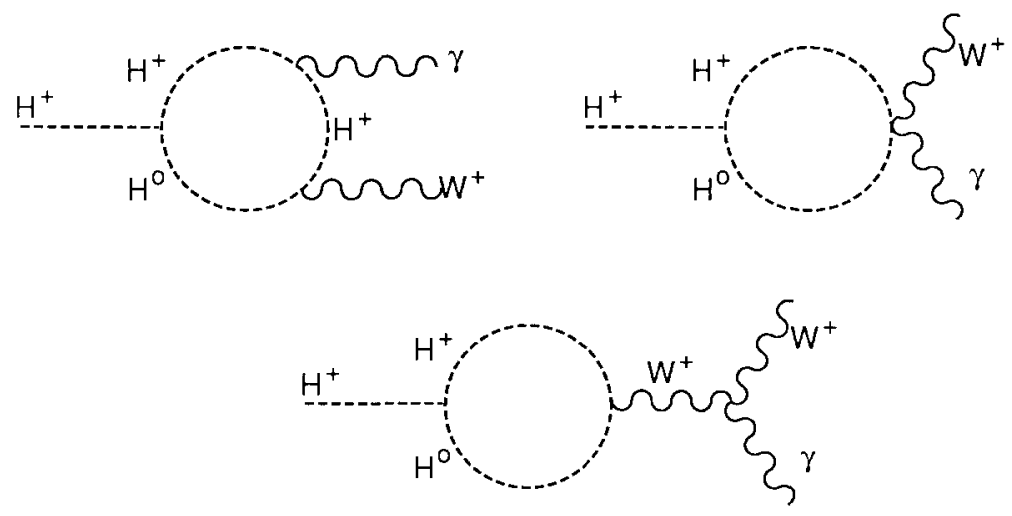

(d)

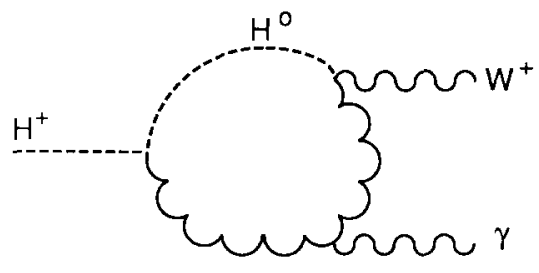

$w^{+}$

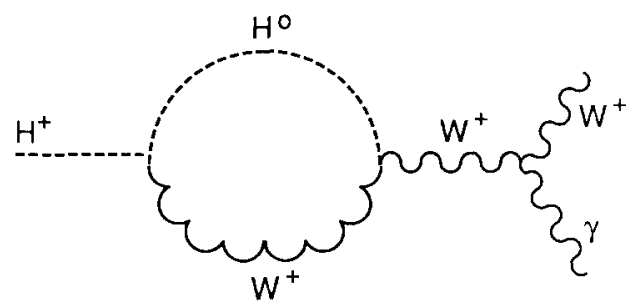

$w^{+}$

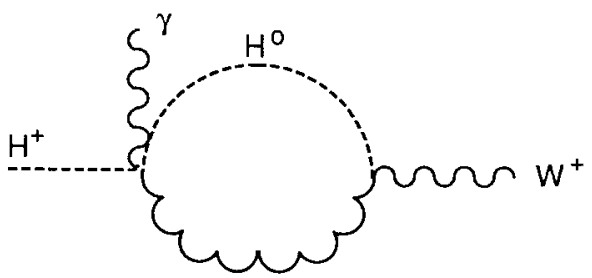

$\mathrm{W}^{+}$

(e)

Fig. 14 (continued).

one $\mathrm{C}$ violating vertex in the fermion loops to first order. For the $\mathrm{H}^{+} \rightarrow \mathrm{W}^{+} \gamma$ case, similar arguments cannot be made since $\mathrm{H}^{+}$is not a $\mathrm{C}$ eigenstate.

From the considerations above we can write the general form of the amplitude as

$$
\mathscr{M}=\left[\mathscr{A} \frac{k^{\nu} q^{\mu}-k \cdot q g^{\mu \nu}}{m_{\mathrm{W}}^{2}}+i \mathscr{G}^{\mu \nu \alpha \beta} \frac{k_{\alpha} q_{\beta}}{m_{\mathrm{W}}^{2}}\right] \varepsilon_{\mu} \varepsilon_{\nu}^{\prime},
$$

where $\varepsilon, \varepsilon^{\prime}$ are the photon's and W's polarization vectors respectively. Denoting by 
$m_{+}$the mass of the charged Higgs scalar, the corresponding width is given by

$$
\Gamma\left(\mathrm{H}^{+} \rightarrow \mathrm{W}^{+} \gamma\right)=\frac{1}{32 \pi} m_{+}^{3}\left(|\mathscr{A}|^{2}+|\mathscr{G}|^{2}\right)\left(1-\frac{m_{\mathrm{W}}^{2}}{m_{+}^{2}}\right)^{3} .
$$

All the five groups of diagrams contribute to $\mathscr{A}$, but only the fermion loops contribute to $\mathscr{G}$. Before we list each contribution it is convenient to define the auxiliary quantities

$$
\begin{aligned}
F_{n}(m, M) & \equiv \int_{0}^{1} \mathrm{~d} x \cdot x^{n} \ln \left(\frac{m^{2}(1-x)+M^{2} x-x(1-x) m_{+}^{2}}{m^{2}(1-x)+M^{2} x-x(1-x) m_{\mathrm{W}}^{2}}\right), \\
\Delta & \equiv m_{+}^{2}-m_{\mathrm{W}}^{2} .
\end{aligned}
$$

Note that $F_{n}$ may acquire an imaginary part; following the $i \varepsilon$ throughout the calculation shows that one should use $\ln (-y)=\ln |y|-i \pi \theta(y)$ as the proper analytic continuation of the logarithm. Now we list the contribution from each group of diagrams.

1. Fermions. The contributions from one fermion doublet are

$$
\begin{gathered}
\mathscr{A}_{\mathrm{fer}}=-\frac{i g^{2} e}{16 \pi^{2} m_{\mathrm{w}} \Delta}\left\{Q_{\mathrm{u}} m_{\mathrm{u}}^{2} \cot \beta\right. \\
+(1+\eta)+\left(\frac{2 m_{\mathrm{u}}^{2}}{\Delta}(1+\eta)-1\right) F_{-1}\left(m_{\mathrm{u}}, m_{\mathrm{d}}\right) \\
+2\left(\frac{m_{\mathrm{d}}^{2}-m_{\mathrm{u}}^{2}-m_{\mathrm{W}}^{2}}{\Delta}(1+\eta)+\frac{1-\eta}{2}\right) F_{0}\left(m_{\mathrm{u}}, m_{\mathrm{d}}\right) \\
\left.+2(1+\eta) \frac{m_{\mathrm{W}}^{2}}{\Delta} F_{1}\left(m_{\mathrm{u}}, m_{\mathrm{d}}\right)\right] \\
+Q_{\mathrm{d}} m_{\mathrm{d}}^{2} \tan \beta\left[\left(1+\frac{1}{\eta}\right)+\left(\frac{2 m_{\mathrm{d}}^{2}}{\Delta}\left(1+\frac{1}{\eta}\right)-1\right) F_{-1}\left(m_{\mathrm{d}}, m_{\mathrm{u}}\right)\right. \\
+2\left(\frac{m_{\mathrm{u}}^{2}-m_{\mathrm{d}}^{2}-m_{\mathrm{W}}^{2}}{\Delta}\left(1+\frac{1}{\eta}\right)+\frac{1-1 / \eta}{2}\right) F_{0}\left(m_{\mathrm{d}}, m_{\mathrm{u}}\right) \\
\left.\left.+2\left(1+\frac{1}{\eta}\right) \frac{m_{\mathrm{W}}^{2}}{\Delta} F_{\mathrm{l}}\left(m_{\mathrm{d}}, m_{\mathrm{u}}\right)\right]\right\}
\end{gathered}
$$$$
\mathscr{G}_{\text {fer }}=-\frac{i g^{2} e}{16 \pi^{2} m_{\mathrm{w}} \Delta}\left\{Q_{\mathrm{u}} m_{\mathrm{u}}^{2} \cot \beta\left[F_{-1}\left(m_{\mathrm{u}}, m_{\mathrm{d}}\right)-(1+\eta) F_{0}\left(m_{\mathrm{u}}, m_{\mathrm{d}}\right)\right]\right.
$$$$
\left.+Q_{\mathrm{d}} m_{\mathrm{d}}^{2} \tan \beta\left[F_{-1}\left(m_{\mathrm{d}}, m_{\mathrm{u}}\right)-(1+1 / \eta) F_{0}\left(m_{\mathrm{d}}, m_{\mathrm{u}}\right)\right]\right\},
$$ 
where $m_{\mathrm{u}}$ and $m_{\mathrm{d}}$ are the up-quark and down-quark masses of the generation being considered, and $\eta \equiv\left(m_{\mathrm{d}}(\tan \beta) / m_{\mathrm{u}}\right)^{2}$. In all the calculations we neglect the effects of the Kobayashi-Maskawa matrix.

2. Charginos and neutralinos. Let $\tilde{m}_{0 i}$ and $\tilde{m}_{j}$ denote the neutralino and chargino masses respectively and, in the notation of refs. [24] and [47],

$$
\begin{aligned}
v_{i j}^{\mathrm{LR}} & \equiv\left(Q_{i j}^{\prime \mathrm{L}}\right) * O_{i j}^{\mathrm{R}}, \\
v_{i j}^{\mathrm{RL}} & \equiv\left(Q_{i j}^{\prime \mathrm{R}}\right) * O_{i j}^{\mathrm{L}}, \\
v_{i j}^{\mathrm{LL}} & \equiv\left(Q_{i j}^{\prime \mathrm{L}}\right) * O_{i j}^{\mathrm{L}}, \\
v_{i j}^{\mathrm{RR}} & \equiv\left(Q_{i j}^{\prime \mathrm{R}}\right) * O_{i j}^{\mathrm{R}},
\end{aligned}
$$

where there is no sum over the indices; $i=1, \ldots, 4 ; j=1,2$. The contributions to the amplitude are then

$$
\begin{aligned}
& \mathscr{A}_{\text {ino }}=\frac{i g e}{8 \pi^{2} \Delta} \sum_{j=1}^{2} \sum_{i=1}^{4}\{-\left[\tilde{m}_{j}\left(v_{i j}^{\mathrm{LR}}+v_{i j}^{\mathrm{RL}}\right)+\frac{\tilde{m}_{0 i}}{3}\left(v_{i j}^{\mathrm{LL}}+v_{i j}^{\mathrm{RR}}\right)\right] \\
&-\tilde{m}_{j}\left(v_{i j}^{\mathrm{LR}}+v_{i j}^{\mathrm{RL}}\right)\left(-1+\frac{2 \tilde{m}_{j}^{2}}{\Delta}\right) F_{-1}\left(\tilde{m}_{j}, \tilde{m}_{0 i}\right) \\
&+\left[\tilde{m}_{0 i}\left(v_{i j}^{\mathrm{RR}}+v_{i j}^{\mathrm{LL}}\right)\left(1-\frac{2 \tilde{m}_{j}^{2}}{\Delta}\right)\right. \\
&-2 \tilde{m}_{j}\left(v_{i j}^{\mathrm{LR}}+v_{i j}^{\mathrm{RL}}\right)\left(\frac{1}{2}+\frac{\left.\left.\tilde{m}_{0 i}^{2}-\tilde{m}_{j}^{2}-m_{\mathrm{W}}^{2}\right)\right] F_{0}\left(\tilde{m}_{j}, \tilde{m}_{0 i}\right)}{\Delta}\right. \\
&-2\left[\tilde{m}_{0 i}\left(v_{i j}^{\mathrm{LL}}+v_{i j}^{\mathrm{RR}}\right) \frac{\tilde{m}_{0 i}^{2}-\tilde{m}_{j}^{2}-m_{\mathrm{W}}^{2}}{\Delta}\right. \\
&\left.+\left\{\tilde{m}_{0 i}\left(v_{i j}^{\mathrm{LL}}-v_{i j}^{\mathrm{RR}}\right)-\tilde{m}_{j}\left(v_{i j}^{\mathrm{LR}}-v_{i j}^{\mathrm{RL}}\right)\right\} F_{0}\left(\tilde{m}_{j}, \tilde{m}_{0 i}\right)\right] . \\
&+\mathscr{G}_{\text {ino }}=\frac{\tilde{m}_{j}\left(v_{i j}^{\mathrm{LR}}+v_{i j}^{\mathrm{RL}}\right) \frac{m_{\mathrm{W}}^{2}}{8 \pi^{2} \Delta}\left[\tilde{m}_{j}\left(v_{i j}^{\mathrm{LR}}-v_{i j}^{\mathrm{RL}}\right) F_{-1}\left(\tilde{m}_{j}, \tilde{m}_{0 i}\right)\right.}{(\mathrm{A} .10)} \\
&\left.\quad-2 \tilde{m}_{0 i}\left(v_{i j}^{\mathrm{LL}}+v_{i j}^{\mathrm{RR}}\right) \frac{m_{\mathrm{W}}^{2}}{\Delta} F_{2}\left(\tilde{m}_{j}, \tilde{m}_{0 i}\right)\right\},
\end{aligned}
$$


3. Scalar-fermion loops. The contribution from one up-down doublet of masses $\tilde{m}_{\mathrm{u}}, \tilde{m}_{\mathrm{d}}$ is given by

$$
\begin{aligned}
\mathscr{A}_{\mathrm{s}-\mathrm{fermions}}= & \frac{i g^{2} e m_{\mathrm{W}}\left(\frac{m_{\mathrm{d}}^{2} \tan \beta+m_{\mathrm{u}}^{2} \cot \beta}{16 \pi^{2} \Delta^{2}}-\sin 2 \beta\right)}{m_{\mathrm{W}}^{2}} \\
& \times\left\{Q _ { \mathrm { u } } \left[\Delta+2 \tilde{m}_{\mathrm{u}}^{2} F_{-1}\left(\tilde{m}_{\mathrm{u}}, \tilde{m}_{\mathrm{d}}\right)+2\left(\tilde{m}_{\mathrm{d}}^{2}-\tilde{m}_{\mathrm{u}}^{2}-m_{\mathrm{W}}^{2}\right) F_{0}\left(\tilde{m}_{\mathrm{u}} \cdot \tilde{m}_{\mathrm{d}}\right)\right.\right. \\
& \left.+2 m_{\mathrm{W}}^{2} F_{1}\left(\tilde{m}_{\mathrm{u}}, \tilde{m}_{\mathrm{d}}\right)\right] \\
& +Q_{\mathrm{d}}\left[\Delta+2 \tilde{m}_{\mathrm{d}}^{2} F_{-1}\left(\tilde{m}_{\mathrm{d}}, \tilde{m}_{\mathrm{u}}\right)+2\left(\tilde{m}_{\mathrm{u}}^{2}-\tilde{m}_{\mathrm{d}}^{2}-m_{\mathrm{W}}^{2}\right) F_{0}\left(\tilde{m}_{\mathrm{d}} \cdot \tilde{m}_{\mathrm{u}}\right)\right. \\
& \left.\left.\quad+2 m_{\mathrm{W}}^{2} F_{1}\left(\tilde{m}_{\mathrm{d}}, \tilde{m}_{\mathrm{u}}\right)\right]\right\}, \quad(\mathrm{A}
\end{aligned}
$$

where $m_{\mathrm{u}}, m_{\mathrm{d}}$ are the masses of the fermionic superpartners of the scalar fermions in the loop.

4. Higgs loops. Let us define the coupling constants

$$
\begin{aligned}
& \lambda_{1} \equiv \sin (\alpha-\beta), \\
& \lambda_{2} \equiv \cos (\alpha-\beta), \\
& \theta_{1} \equiv m_{\mathrm{W}} \cos (\alpha-\beta)-\frac{m_{\mathrm{Z}}}{2 c_{\mathrm{W}}} \cos 2 \beta \cos (\alpha+\beta), \\
& \theta_{2} \equiv-m_{\mathrm{W}} \sin (\alpha-\beta)+\frac{m_{\mathrm{Z}}}{2 c_{\mathrm{W}}} \cos 2 \beta \sin (\alpha+\beta),
\end{aligned}
$$

and let $m_{i}^{0},(i=1,2)$ denote the neutral Higgs masses that contribute to the loop. After some calculation we obtain

$$
\begin{aligned}
\mathscr{A}_{\mathrm{Higgs}}=\frac{i g^{2} e}{32 \pi^{2} \Delta^{2}} & \sum_{i=1}^{2} \theta_{i} \lambda_{i}\left[\Delta+2 m_{+}^{2} F_{-1}\left(m_{+}, m_{i}^{0}\right)\right. \\
& \left.+2\left(\left[m_{i}^{0}\right]^{2}-m_{+}^{2}-m_{\mathrm{W}}^{2}\right) F_{0}\left(m_{+}, m_{i}^{0}\right)+2 m_{\mathrm{W}}^{2} F_{1}\left(m_{+}, m_{i}^{0}\right)\right] .
\end{aligned}
$$

5. W-Higgs loops. This is the contribution most difficult to evaluate and it is here that the complications of the unitary gauge appear with a vengeance. The second and third diagrams in this group can be evaluated without too much problem, but for the first one it is necessary to use an algebraic manipulation program to obtain the correct answer in a reasonable time; we used REDUCE. The 
most important check of the final result is, of course, gauge invariance. After a very long and tedious calculation we find

$$
\begin{aligned}
\mathscr{A}_{\mathrm{W}-\mathrm{H}}= & \frac{i g^{2} e}{64 \pi^{2} m_{\mathrm{W}} \Delta^{2}} \sum_{i=1}^{2} \sigma_{i} \lambda_{i} \\
\times & {\left[-\frac{7}{3} \Delta\left[m_{i}^{0}\right]^{2}+4 m_{+}^{2}\left(m_{+}^{2}-2 m_{\mathrm{W}}^{2}\right) F_{3}\left(m_{\mathrm{W}}, m_{i}^{0}\right)\right.} \\
& -6\left(m_{+}^{4}-2 m_{+}^{2} m_{\mathrm{W}}^{2}-m_{\mathrm{W}}^{4}+m_{\mathrm{W}}^{2}\left[m_{i}^{0}\right]^{2}\right) F_{2}\left(m_{\mathrm{W}}, m_{i}^{0}\right) \\
& +\left(m_{+}^{4}-3 m_{\mathrm{W}}^{4}+2 m_{\mathrm{W}}^{2}\left[m_{i}^{0}\right]^{2}-\left[m_{i}^{0}\right]^{4}\right) F_{1}\left(m_{\mathrm{W}}, m_{i}^{0}\right) \\
& +\left(2 m_{+}^{2}\left[m_{i}^{0}\right]^{2}-6 m_{\mathrm{W}}^{4}+5 m_{\mathrm{W}}^{2}\left[m_{i}^{0}\right]^{2}-2\left[m_{i}^{0}\right]^{4}\right) F_{0}\left(m_{\mathrm{W}}, m_{i}^{0}\right) \\
& -2 m_{\mathrm{W}}^{2}\left(m_{+}^{2}-3 m_{\mathrm{W}}^{2}+\left[m_{i}^{0}\right]^{2}\right) F_{-1}\left(m_{\mathrm{W}}, m_{i}^{0}\right) \\
& -\Delta^{2} \frac{\left[m_{i}^{0}\right]^{2}}{m_{\mathrm{W}}^{2}}\left(\frac{\left[m_{i}^{0}\right]^{4}}{m_{\mathrm{W}}^{4}}+\frac{3}{2} \frac{\left[m_{i}^{0}\right]^{2}}{m_{\mathrm{W}}^{2}}+\frac{1}{3}\right) \\
& +\Delta^{2} \frac{\left[m_{i}^{0}\right]^{4}}{m_{\mathrm{W}}^{4}}\left(1+\frac{\left[m_{i}^{0}\right]^{2}}{m_{\mathrm{W}}^{2}}\right)^{2}\left(\frac{m_{\mathrm{W}}}{\left[m_{i}^{0}\right]^{2}}+1\right), \\
& (A)
\end{aligned}
$$

where $\sigma_{1} \equiv \lambda_{2}, \sigma_{2} \equiv-\lambda_{1}$, and $m_{1}^{0}, m_{2}^{0}$ are the masses of the heavy and light neutral scalar Higgs bosons, respectively $(\mathrm{H}$ and $\mathrm{h}$ in the notation of the main text).

This concludes the list of contributions to the two form factors mentioned above.

If one calculates the value for $\Gamma\left(\mathrm{H}^{+} \rightarrow \mathrm{W}^{+} \gamma\right)$ in the context of the broken supersymmetric model considered in refs. [24] and [47], one finds the rather disappointing result that the values for the branching ratio for this mode are never higher than $\sim 5 \times 10^{-5}$, and usually lower. In addition, it appears that only the leptonic decays of the $W$ will be useful due to background problems. The width for $\mathrm{H}^{+} \rightarrow \mathrm{W}^{+} \gamma$ with the $\mathrm{W}$ decaying into $\mathrm{e} \nu_{\mathrm{e}}$ or $\mu \nu_{\mu}$ is (depending on $m_{\mathrm{t}}$ )

$$
\sum_{1=\mathrm{e}, \mu} \Gamma\left(\mathrm{H}^{+} \rightarrow \mathrm{W}^{+} \gamma \rightarrow \ell \nu_{\ell} \gamma\right) \sim 0.2 \Gamma\left(\mathrm{H}^{+} \rightarrow \mathrm{W}^{+} \gamma\right)
$$

The smallness of the final result, as compared to $\Gamma\left(\mathrm{H}_{\mathrm{SM}}^{0} \rightarrow \mathrm{Z} \gamma\right)$, is due to strong cancellations between fermion and boson loops, and between the two neutral Higgs bosons which have opposite sign coupling constants. Another important fact is the 
strong correlation between the charged Higgs mass, $m_{+}$, and that of the heavy neutral scalar, $m_{1}^{0}$. (For this reason a small branching ratio persists even if we decouple the scalar-fermions and the charginos and neutralinos.) Notice that $\Gamma\left(\mathrm{H}^{+} \rightarrow \mathrm{W}^{+} \gamma\right)$ is very sensitive to the value of $\beta$. For example, the fermion loops give a very large contribution as $\beta \rightarrow \frac{1}{2} \pi$. However this also happens in the decay $\mathrm{H}^{+} \rightarrow \mathrm{t} \overline{\mathrm{b}}$ so that the branching ratio is still small in this limit.

If one goes outside the framework of the above mentioned supersymmetric model, all parameters in the formulas become free and there are cases where we do find large branching ratios $\left(\sim 10^{-4}\right.$ or larger). However, it should be stressed that a thorough determination of the regions in parameter space that correspond to a large branching ratio is hampered by the number of unknowns: disregarding the constraints coming from broken supersymmetry we have (ignoring the KobayashiMaskawa matrix and assuming three families) 88 free parameters.

As an example, a way of obtaining such a large branching ratio is by assuming that $m_{+} \ll m_{1}^{0}$ (in the model we considered, these two masses are strongly correlated and almost equal). We have also studied the effects from an extra heavy W, which turned out to be small. The inclusion of a heavy fermion doublet also gives a small contribution which, however, is mildly dependent on the mass difference of the doublet.

\section{References}

[1] M. Veltman, Scientific American (November 1986) p. 76

[2] J. Boucrot et al., Search for neutral Higgs at LEP-200, CERN-EP/87-40, ECFA Workshop (February, 1987)

[3] E. Wang et al., in Proc. 1987 Workshop on Experiments, Detectors, and Experimental Areas for the Super Collider, ed. R. Donaldson and M.G.D. Gilchriese;

F. Paige and E. Wang, in Proc. of the 1987 Madison Workshop on From Colliders to Super Colliders

[4] J.F. Gunion and H.E. Haber, Probing the Higgs sector: the standard model and beyond, in Proc. 1987 Madison Workshop on From Colliders to Super Colliders, p. 67; Int. J. Mod. Phys. (1987) 957

[5] M. Veltman, Acta Phys. Pol. B8 (1977) 475;

B.W. Lee, C. Quigg, and H. Thacker, Phys. Rev. D16 (1977) 1519;

M.S. Chanowitz and M.K. Gaillard, Nucl. Phys. B261 (1985) 379;

M.J. Duncan, G.L. Kane, and W.W. Repko, Nucl. Phys. B272 (1986) 517

[6] D.R.T. Jones and S.T. Petkov, Phys. Lett. 136B (1979) 440;

R.N. Cahn and S. Dawson, Phys. Lett. 136B (1984) 196;

G.L. Kane, W.W. Repko, and W.B. Rolnick, Phys. Lett. 148B (1984) 367;

M.S. Chanowitz and M.K. Gaillard, Phys. Lett. 142B (1984) 85;

S. Dawson, Nucl. Phys. B249 (1985) 42

[7] See the last two papers of ref. [5]

[8] J.F. Gunion, J. Kalinowski, and A. Tofighi-Niaki, Phys. Rev. Lett. 57 (1986) 2351;

J.F. Gunion, J. Kalinowski, A. Tofighi-Niaki, A. Abbasabadi, and W. Repko, Proc. of the 1986 Snowmass Workshop on the Design and Utilization of the Superconducting Super Collider, ed. R. Donaldson and J. Marx, p. 156;

J.F. Gunion and A. Tofighi-Niaki, Phys. Rev. D36 (1987) 2671

[9] D.A. Dicus and R. Vega, Phys. Rev. Lett 57 (1986) 1110;

D.A. Dicus, S.L. Wilson, and R. Vega, Phys. Lett. 192B (1987) 231 
[10] J.F. Gunion, Z. Kunszt, and M. Soldate, Phys. Lett. 163B (1985) 389; (E) 168B (1986) 427

[11] W.J. Stirling, R. Kleiss, and S.D. Ellis, Phys. Lett. B163 (1985) 261

[12] J.F. Gunion and M. Soldate, Phys. Rev. D34 (1986) 826

[13] R.N. Cahn, S.D. Ellis, R. Kleiss, and W.J. Stirling, Proc. 1986 UCLA Workshop on Observable Standard Model Physics at the SSC, ed. H.-U. Bengtsson, C. Buchanan, T. Gottschalk, and A. Soni, p. 102; and preprint LBL-21649 (1986)

[14] J.F. Gunion. Proc. 1986 Snowmass Workshop on the Design and Utilization of the Superconducting Super Collider, ed. R. Donaldsoin and J. Marx, p. 412; and ref. [4]

[15] G. Alverson et al., Proceedings of the 1986 Snowmass Workshop on the Design and Utilization of the Superconducting Super Collider, ed. R. Donaldson and J. Marx, pp. 93, 114; ref. [3]; and the forthcoming SLAC studies on future $\mathrm{e}^{+} \mathrm{e}^{-}$colliders. Parallel studies have been done in Europe as part of the La Thuile workshop: see contributions by D. Froidevaux et al.

[16] J.F. Gunion and A. Tofighi-Niaki, third paper of ref. [8]

[17] J.F. Gunion, P. Kalyniak, M. Soldate, and P. Galison, Phys. Rev. Lett. 54 (1985) 1226

[18] J.F. Gunion, P. Kalyniak, M. Soldate, and P. Galison, Phys. Rev. D34 (1986) 101

[19] B. Cox and F.J. Gilman, Proc. 1984 Snowmass Workshop on the Design and Utilization of the Superconducting Super Collider, ed. R. Donaldson and J.G. Morfin, p. 87

[20] L. Price and F.J. Gilman, Proc. 1986 Snowmass Workshop on the Design and Utilization of the Superconducting Super Collider, ed. R. Donaldson and J. Marx, p. 195

[21] G. Altarelli and E. Franco, Mod. Phys. Lett. A1 (1986) 517

[22] J.F. Gunion and G. Kane, The case for a high luminosity interaction region at the SSC, in Proc. 1986 Snowmass Workshop on the Design and Utilization of the Superconducting Super Collider, ed. R. Donaldson and J. Marx

[23] D.W. Dusedau and J. Wudka, Phys. Lett. B180 (1986) 290

[24] J.F. Gunion and H.E. Haber, Nucl. Phys. B272 (1986) 1

[25] J.F. Gunion and H.E. Haber, Nucl. Phys. B278 (1986) 449

[26] H.E. Haber, I. Kani, G.L. Kane, and M. Quif́os, Nucl. Phys. B283 (1987) 111

[27] J. Ellis, M.K. Gaillard, and D. Nanopoulos, Nucl. Phys. B106 (1976) 292

[28] A.I. Vainstein, M.B. Voloshin, V.I. Zakharov, and M.A. Shifman, Sov. J. Nucl. Phys. 30 (1979) 711

[29] R. Bates, J.N. Ng, and P. Kalyniak, Phys. Rev. 34 (1986) 172

[30] R. Cahn, M.C. Chanowitz, and N. Fleishon, Phys. Lett. 82B (1979) 113

[31] R. Baier and R. Ruckl, Z. Phys. C19 (1983) 251

[32] M.I. Vysotsky, Phys. Lett. 97B (1980) 159

[33] W.Y Keung and W.J. Marciano, Phys. Rev. D30 (1984) 248

[34] J.F. Gunion, H.E. Haber, F.E. Paige, Wu-Ki Tung, and S.S.D. Willenbrock, Nucl. Phys. B294 (1987) 621

[35] C. Baltay, J. Huston, and B.G. Pope, Calorimetry for SSC detectors; D. Carlsmith et al., SSC muon detector group report, Proc. 1986 Snowmass Workshop on the Design and Utilization of the Superconducting Super Collider, ed. R. Donaldson and J. Marx, pp. 355, 405; C. Baltay, private communication

[36] Ll. Amteller, E. Gava, N. Paver, and D. Treleani, Phys. Rev. D32 (1985) 1699

[37] I. Bigi, Y. Dokshitzer, V. Khoze, J. Kuhn, and P. Zerwas, Phys. Lett. 181 (1986) 157

[38] Y. Morita, p. 194, and S. Mikawa and A. Yamashita, Proc. 1986 Snowmass Workshop on the Design and Utilization of the Superconducting Super Collider, ed. R. Donaldson and J. Marx, p. 278

[39] Proc. 1987 Workshop on Experiments, Detectors, and Experimental Ateas for the Super Collider. ed. R. Donaldson and M.G.D. Gilchriese

[40] A. Appel et al., Phys. Lett. 176B (1986) 239

[41] J.F. Gunion and J. Lewis, in preparation

[42] D. Smith, private communication

[43] D. Atwood and J.F. Gunion, in preparation. See also ref. [4] for a summary.

[44] K. Ellis, I. Hinchliffe, M. Soldate, and J.J. van der Bij, preprint FERMILAB-PUB-87/100-T

[45] S. Komamiya, SLAC Linear Collider Study Group Report

[46] The CELLO group, preprint DESY-87-030 (April, 1987)

[47] H.E. Haber and G.L. Kane, Phys. Reports 117 (1985) 75 
[48] J.A. Grifols and A. Mendez, Phys. Rev. D22 (1980) 1725;

J.L. Diaz-Cruz and M.A. Perez, Phys. Rev. D33 (1986) 273

[49] J. Grifols, J.F. Gunion, and A. Mendez, Phys, Lett., B197 (1987) 266

[50] J.F. Gunion and D. Millers, work in progress

[51] H.-U. Bengtsson, S. Komamiya and H. Yamamoto, Proc. 1987 Madison Workshop on From Colliders to Super Colliders, p. 165

[52] Probing the non-minimal Higgs sector at the SSC, in Proc. 1987 Workshop on Experiments, Detectors, and Experimental Areas for the Super Collider, ed. R. Donaldson and M.G.D. Gilchriese, and references therein

[53] R.W. Brown, D. Sahdev, and K.O. Mikaelian, Phys. Rev. D20 (1979) 1164

[54] E. Eichten, I. Hinchliffe, K. Lane, and C. Quigg, Rev. Mod. Phys. 56 (1984) 579

[55] J.F. Gunion et al., Proc. 1986 Snowmass Workshop on the Design and Utilization of the Superconducting Super Collider, ed. R. Donaldson and J. Marx, p. 103; ref. [19]; and ref. [20]

[56] Z. Kunszt et al., Proc. La Thuile Study on Future Colliders (January, 1987)

[57] J.F. Gunion, H.E. Haber et al., Proc. 1987 Madison Workshop on From Colliders to Super Colliders, p. 145; Int. J. Mod. Phys. (1987) 1035

[58] R.M. Barnett, J.A. Grifols, A. Mendez, J.F. Gunion, and J. Kalinowski, Detection of a heavy neutral Higgs boson in a higgsino-neutralino decay mode, in Proc. 1986 Snowmass Workshop on the Design and Utilization of the Superconducting Super Collider, ed. R. Donaldson and J. Marx, p. 188 